\title{
SU(3) つ R(3) WIGNER COEFFICIENTS IN THE 2s-1d SHELL
}

\author{
J. D. VERGADOS
}

Physics Department, The Unit ersity of Michigan, Ann Arbor, Michigan ${ }^{\dagger}$

\author{
Received 2 October 1967
}

\begin{abstract}
Explicit algebraic expressions are given for $S U(3) \supset R(3)$ Wigner coefficients, which are of particular interest in p-shell and $2 \mathrm{~s}-1 \mathrm{~d}$ shell-model calculations. An orthogonal basis tied closely to Elliott's basis is chosen according to a systematic recipe. The Wigner coefficients are those involving the Kronecker products $\left(\lambda_{1} \mu_{1}\right) \times\left(\lambda_{2} \mu_{3}\right) \rightarrow\left(\lambda_{3} \mu_{3}\right)$ in the following cases:

(i) $\left(\lambda_{2} \mu_{2}\right)=(10),(01)$ and $\mu_{1} \leqq 4$ (arbitrary $\left.\lambda_{2}\right), i=1,3$. (For the case $\mu_{i}=4$, only auxiliary coefficients are tabulated whenever the angular momentum multiplicity is 3 . From these the final Wigner coefficients can be calculated for any specific case of interest.)

(ii) $\left(\lambda_{2} \mu_{2}\right)=(20),(02)$ and $\mu_{1} \leqq 3\left(\right.$ arbitrary $\left.\lambda_{i}\right)$, or $\lambda_{1} \leqq 3\left(\right.$ arbitrary $\left.\mu_{1}\right) i=1,3$.

(iii) $\left(\lambda_{2} \mu_{2}\right)=(11)$ and $\mu_{2} \leqq 3$ (arbitrary $\lambda_{1}$ ), or $\lambda_{2} \leqq 3$ (arbitrary $\mu_{1}$ ) $i=1,3$ except for $($ j.3 $) \times(11) \rightarrow(\lambda 3)$ which is not given.

A method is also given whereby coefficients with more complicated $\left(\lambda_{2} \mu_{2}\right)$ can be calculated from coefficients of type (i) by a build-up process. Some symmetry properties are discussed.
\end{abstract}

\section{Introduction}

The importance of the group SU(3) for shell-model calculations has been recognized by Elliott ${ }^{1,2}$ ) and exploited by many authors ${ }^{2-8}$ ). The states of interest are the harmonic oscillator states, which form a basis for the irreducible representations of SU(3). To completely specify the states one seeks to construct a complete set of commuting operators based on the invariants of a canonical chain of subgroups. The conventional and mathematically "natural" chain is Gelfand's chain ${ }^{9-11}$ ) of unitary groups, specifically here $\mathrm{SU}(3) \supset \mathrm{SU}(2)$. For $\mathrm{SU}(3)$ a detailed solution of this problem is known ${ }^{3,12,13}$ ).

In nuclear physics, however, SU(3) serves partly to characterize the symmetry of the orbital part of the wave function. Therefore the SU(3) states needed for shellmodel calculations must be states of good angular momentum, that is states based on the chain of subgroups $S U(3) \supset R(3) \supset R(2)$. Bargmann and Moshinsky ${ }^{14}$ ) have introduced an operator, which together with the Casimir invariants of the above chain of subgroups, completely characterizes the states of an irreducible representation of SU(3). In practice, however, it is very difficult to work with this basis in actual shell-model calculations.

+ Work supported in part by the U.S. Atomic Energy Commission. 
An alternate and more useful technique has been employed by Elliott ${ }^{1,2}$ ), who constructs good angular momentum states by projecting them out of a single SU(3) state. In cases where the angular momentum quantum numbers $L, M$ do not suffice to completely specify the states that occur in a given $\operatorname{SU}(3)$ representation $(\lambda, \mu)$, Elliott's projection technique naturally furnishes an extra label $K$ which serves to completely classify the states. In addition, the label $K$ turns out to have real physical significance, since it can be interpreted to characterize the different rotational bands in a given SU(3) representation. However, it is not possible to make a direct application of the elegant Racah algebra in this classification scheme, since the Elliott basis states do not form an orthogonal set. States of a given representation with the same angular momentum quantum numbers and different values of $K$ are not orthogonal to each other.

To apply Racah techniques in the 2s-1d shell one has to know the $\mathrm{SU}(3) \supset \mathrm{R}(3)$ Wigner coefficients involved. Engeland ${ }^{4}$ ) has given an expression for the expansion coefficients $C\left(\left(\lambda_{1} \mu_{1}\right) K_{1} L_{1} M_{1},\left(\lambda_{2} \mu_{2}\right) K_{2} L_{2} M_{2} ;(\lambda \mu) K L M\right)$ which express the states of the coupled representation $(\lambda \mu)$ in terms of the uncoupled representations in the case of simply reducible products $\left(\lambda_{1} \mu_{1}\right) \times\left(\lambda_{2} \mu_{2}\right)$, namely

$$
\begin{aligned}
|(\lambda \mu) K L M\rangle= & \sum_{\substack{K_{1} K_{2} \\
L_{1} L_{2} \\
M_{1}\left(M_{2}\right)}} C\left(\left(\lambda_{1} \mu_{1}\right) K_{1} L_{1} M_{1},\left(\lambda_{2} \mu_{2}\right) K_{2} L_{2} M_{2} ;(\lambda \mu) K L M\right) \\
& \times\left|\left(\lambda_{1} \mu_{1}\right) K_{1} L_{1} M_{1}\right\rangle\left|\left(\lambda_{2} \mu_{2}\right) K_{2} L_{2} M_{2}\right\rangle .
\end{aligned}
$$

Since the Elliott basis states $|(\lambda \mu) K L M\rangle$ do not form orthogonal sets, Engeland's expansion coefficients $C$ are not the elements of a unitary transformation matrix from an uncoupled to a coupled basis. Nevertheless Engeland's formula is very useful and will be used in the present work to construct the needed $S U(3) \supset R(3)$ Wigner coefficients.

Horie $^{15}$ ) has given algebraic expressions for the simplest $S U(3) \supset R(3)$ Wigner coefficients for the products of the type $\left(\lambda_{1} \mu_{1}\right) \times(10) \rightarrow\left(\lambda_{3} \mu_{3}\right)$. They are basic to any calculation. Unfortunately Horie had to confine himself to $\mu_{1}, \mu_{3} \leqq 2$. In addition, his choice of orthogonal basis in those cases where $L$ and $M$ do not suffice to completely label the states of a given representation is completely arbitrary, therefore it would be difficult to generalize his work in a systematic way.

Recently Akiyama ${ }^{16}$ ) has calculated the $\mathrm{SU}(3) \supset \mathrm{R}(3)$ Wigner coefficients needed for the one-particle c.f.p. for the 2 s-1d shell with $n \leqq 6$. However, his method of calculation and his results are entirely numerical and therefore difficult to extend to large SU(3) quantum numbers. His choice of orthogonal states in a given $(\lambda \mu)$, when there is multiplicity in $L$, is again completely arbitrary.

In the present work an attempt is made to give algebraic expressions for most, if not all, of the $S U(3) \supset R(3)$ Wigner coefficients needed for calculations in the $2 s-1 d$ shell. The orthogonal states of a given irreducible representation are constructed in a systematic and uniform way for all $(\lambda \mu)$, such that the states are as close as possible 
to Elliott's physically meaningful basis. A Gram-Schmidt ${ }^{17}$ ) orthogonalization procedure is employed to construct an orthonormal basis from the complete set of Elliott's basis.

The calculation of $\mathrm{SU}(3) \supset \mathrm{R}(3)$ Wigner coefficients proceeds into two steps. In the first step the basic or generating coefficients for the product $\left(\lambda_{1} \mu_{1}\right) \times(10) \rightarrow$ $\left(\lambda_{3} \mu_{3}\right)$ are calculated. As a result of the systematic choice of basis vectors, the algebraic structure of these coefficients is somewhat simpler than that of Horie's coefficients. So it is easier to extend the tables to larger SU(3) quantum numbers. Nevertheless the algebraic structure of the coefficients becomes more and more complicated as the multiplicity of $L$ in a given representation increases. It was therefore necessary to confine the tables to representations for which $\mu \leqq 4$ (arbitrary $\lambda$ ), or $\lambda \leqq 4$ (arbitrary $\mu$ ). It is believed that the algebraic complications arise from the requirement that the basis states diagonalize $L^{2}$ and $L_{\mathrm{Z}}$, and that no further clever choice of the needed extra quantum number would substantially reduce these complications in representations in which both $\lambda, \mu \geqq 5$.

The second step in the calculation of Wigner coefficients is a "building-up" process in which the SU(3) Wigner coefficients for products such as $\left(\lambda_{1} \mu_{1}\right) \times(20)$, or $\left(\lambda_{1} \mu_{1}\right) \times(11)$ are constructed from the basic ones calculated in step one. Coefficients for more complicated products can be calculated by a repeated application of the "building-up" process. To apply the building-up process expressions for SU(3) $U$-functions (Racah coefficients) are needed. Most of the necessary $U$-functions have already been tabulated ${ }^{3,18}$ ). A few additional ones are given in table 1. (The $U$ functions depend only on the SU(3) representations involved and not on the particular chain of subgroups chosen.)

In order to make the $S U(3) \supset R(3)$ Wigner coefficients real, a modification was made in Elliott's phase convention retaining, however, the well-established, CondonShortly ${ }^{19}$ ) phase convention for the R(3) part of the coefficients.

It is hoped that, by making the above coefficients available in algebraic form, most of the $2 \mathrm{~s}-1 \mathrm{~d}$ shell calculations can be simplified. In the very few cases not covered by the tables, the present method can be applied for the specific numbers of interest. In addition, the building-up process can easily be translated into a computer program, and computer facilities may be used.

In order to establish the notation the group SU(3) and Elliott's projection technique is briefly discussed in sect. 2 . Our phase convention and orthonormalization process is given in sect. 3. The building-up process is exhibited in sect. 4 . The construction of the generating coefficients for the products $\left(\lambda_{1} \mu_{1}\right) \times(10) \rightarrow\left(\lambda_{3} \mu_{3}\right)$ is accomplished in sect. 5. Application of the building-up process is illustrated in sect. 6 in which the explicit constructions are given for the Wigner coefficients for the products $\left(\lambda_{1} \mu_{1}\right) \times$ $\left(\lambda_{2} \mu_{2}\right) \rightarrow\left(\lambda_{3} \mu_{3}\right)$ with $\left(\lambda_{2} \mu_{2}\right)=(20)$ or (11). SU(3) $\supset \mathrm{R}(3)$ irreducible tensor operators are defined in sect. 7 . Some symmetry properties are given in sect. 8 . They can be used to construct the coefficients with $\left(\lambda_{2} \mu_{2}\right)=(01)$ or $(02)$ from the above. A list of tables follows, and some of the proofs are given in the appendices. 

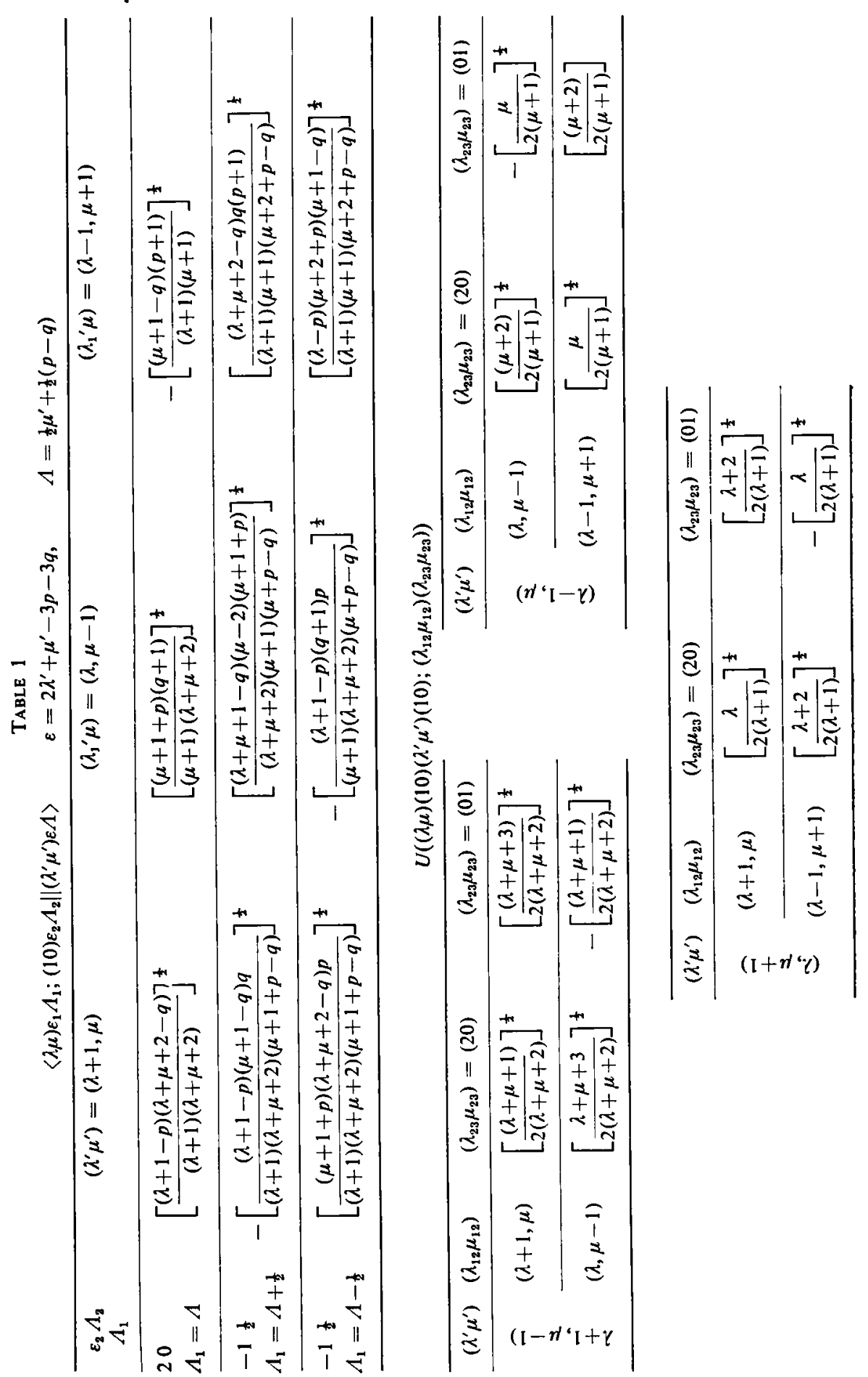

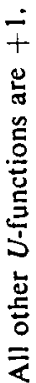




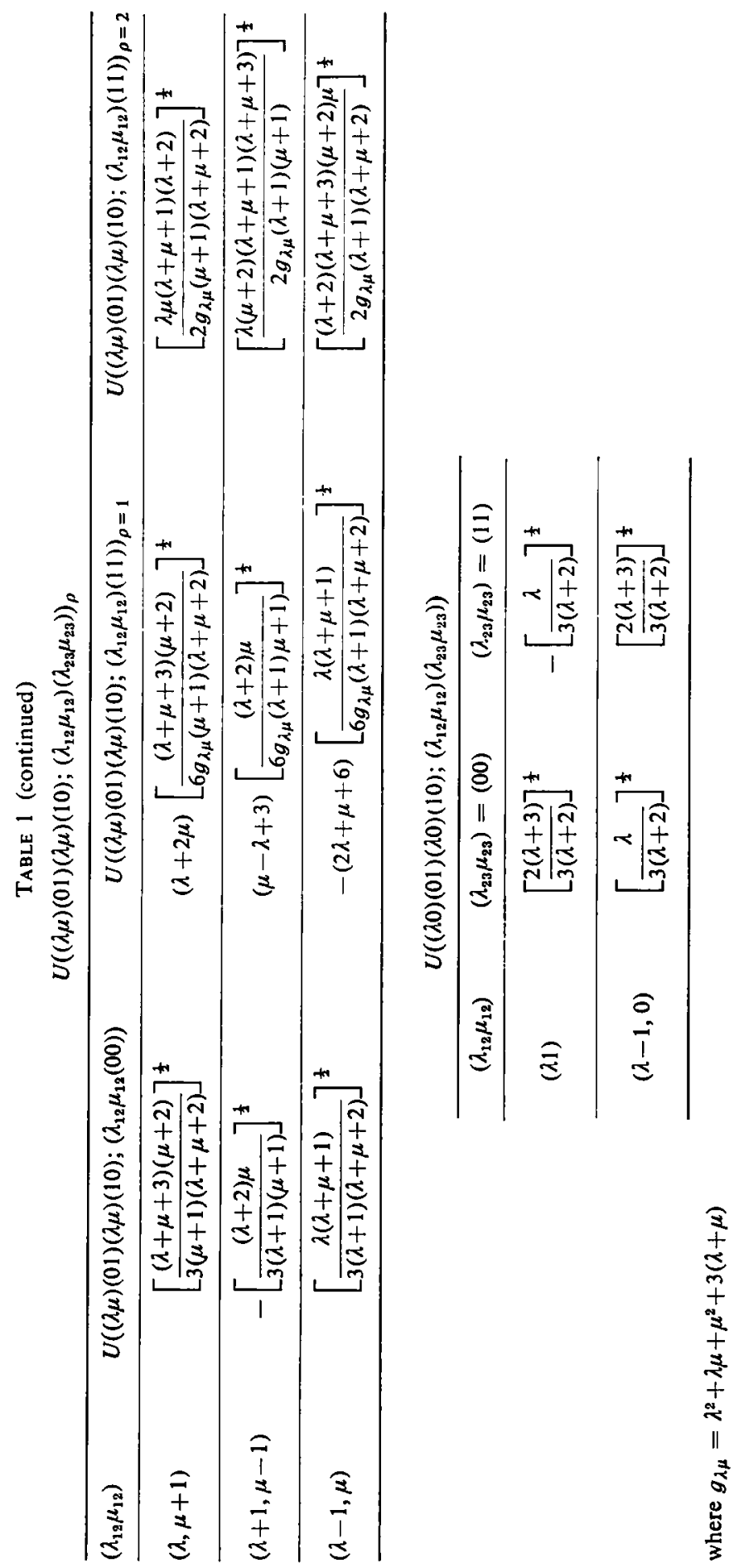




\section{Review of SU(3) and the projection technique}

The notation to be used is essentially that of Elliott and will follow refs. ${ }^{1,2,3,12}$ ). The infinitesimal generators of the SU(3) algebra are denoted by $A_{i j}$ with $i, j=x, y, z$ and commutation relations

$$
\left[A_{i j}, A_{k l}\right]=\delta_{j k} A_{i l}-\delta_{i l} A_{k j} .
$$

The irreducible representations of the SU(3) algebra are denoted by $(\lambda \mu)$ with $\lambda=h_{1}-h_{2}, \mu=h_{2}-h_{3}$, where $\left[h_{1} h_{2} h_{3}\right]$ is a partition of $N$ harmonic oscillator quanta.

In the conventional classification of states that occur in a given representation $(\lambda \mu)$ one chooses the chain $\mathrm{SU}(3) \supset \mathrm{SU}(2)$ and constructs a complete set of commuting operators $\left\{Q_{0}, A_{0}, \Lambda^{2}\right\}$, where $Q_{0}=2 A_{z z}-A_{x x}-A_{y y}, \Lambda_{0}=\frac{1}{2}\left(A_{x x}-A_{y y}\right) ; \Lambda^{2}$ $=\frac{1}{2}\left(A_{x y} A_{y x}+A_{y x} A_{x y}\right)+\Lambda_{0}$. Then a basis $\left|(\lambda \mu) \varepsilon \Lambda \Lambda_{0}\right\rangle$ is defined by

$$
\begin{aligned}
& Q_{0}\left|(\lambda \mu) \varepsilon \Lambda \Lambda_{0}\right\rangle=\varepsilon\left|(\lambda \mu) \varepsilon \Lambda \Lambda_{0}\right\rangle, \\
& \Lambda_{0}\left|(\lambda \mu) \varepsilon \Lambda \Lambda_{0}\right\rangle=\Lambda_{0}\left|(\lambda \mu) \varepsilon \Lambda \Lambda_{0}\right\rangle, \\
& \Lambda^{2}\left|(\lambda \mu) \varepsilon \Lambda \Lambda_{0}\right\rangle=\Lambda(\Lambda+1)\left|(\lambda \mu) \varepsilon \Lambda \Lambda_{0}\right\rangle,
\end{aligned}
$$

where

$$
\varepsilon=2 \lambda+\mu-3(p+q), \quad \Lambda=\frac{1}{2} \mu+\frac{1}{2}(p-q), \quad \Lambda_{0}=\Lambda-r,
$$

with $p, q, r$ integers such that

$$
0 \leqq p \leqq \lambda, \quad 0 \leqq q \leqq \mu, \quad 0 \leqq r \leqq 2 \Lambda .
$$

The states of the basis are constructed by a step-down process starting with the highest-weight state

$$
\left|(\lambda \mu) \varepsilon \Lambda \Lambda_{0}\right\rangle=F(p, q, r)\left|(\lambda \mu) \varepsilon_{H}=2 \lambda+\mu, \quad \Lambda_{H}=\frac{1}{2} \mu, \quad \Lambda_{0 H}=\frac{1}{2} \mu\right\rangle,
$$

where

$$
F(p, q, r)=N((\lambda \mu) p q r) A_{y x}^{r} O_{y z}^{q} A_{x z}^{p},
$$

$N$ is a normalization coefficient calculated by Elliott and Harvey ${ }^{2-4}$ ) and

$$
O_{y z}=A_{y x} A_{x z}-A_{y z}\left(A_{x x}-A_{y y}+1\right) .
$$

States of the above nature will subsequently be called intrinsic states.

Since SU(3) is related to the spatial symmetry of the wave function in shell-model calculations, it is desirable to work with a basis in which the angular momentum is diagonal. For this purpose first a new basis ${ }^{3,12}$ ) for the SU(3) Lie algebra is introduced

where

$$
L_{ \pm}=L_{x} \pm i L_{y}, \quad L_{0}=L_{z}, \quad Q_{0}, \quad Q_{ \pm 1}, \quad Q_{ \pm 2},
$$

$$
\begin{aligned}
L_{x} & =-i\left(A_{y z}-A_{z y}\right), \quad L_{y}=-i\left(A_{z x}-A_{x z}\right), \quad L_{z}=-i\left(A_{x y}-A_{y x}\right), \\
Q_{0} & =2 A_{z z}-A_{y y}-A_{x x}, \\
Q_{ \pm 1} & =\mp \sqrt{\frac{3}{2}}\left[\left(A_{x z}+A_{z x}\right) \pm i\left(A_{y z}+A_{z y}\right)\right], \\
Q_{ \pm 2} & =\sqrt{\frac{3}{2}}\left[\left(A_{x x}-A_{y y}\right) \pm i\left(A_{x y}+A_{y x}\right)\right] .
\end{aligned}
$$


Then a complete set of commuting operators $\left\{\Omega, L^{2}, L_{0}\right\}$ must be constructed, and the new basis should be one in which the above set is diagonal. Bargmann and Moshinsky ${ }^{14}$ ) have succeeded in constructing the operator $\Omega$, but it is difficult to use it in actual calculations. The alternative method originally suggested by Elliott ${ }^{1,2}$ ) is more useful. He showed that any angular momentum state in a given representation can be obtained from the highest weight intrinsic state (i.e. the state with $p=q=r$ $=0$ ) of the same representation

$$
|(\lambda \mu) K L M\rangle=P\left|(\lambda \mu) \varepsilon_{H} \Lambda_{H} \Lambda_{0 H}\right\rangle,
$$

where $P$ is a projection operator given explicitly ${ }^{2}$ ) by

$$
P=\frac{2 L+1}{a(K, L)} \int \mathrm{d} \Omega D_{M K}^{L}(\Omega) R(\Omega),
$$

where $R(\Omega)$ is the rotation operator and the normalization coefficients $a(K, L)$ given by Elliott ${ }^{2}$ ) are

$$
\begin{aligned}
|a(K, L)|^{2}= & A(K L K), \\
A\left(K L K^{\prime}\right)= & \frac{2 L+1}{8 \pi^{2}} \sum_{n=0}^{\mu}\left[\left(\begin{array}{l}
\mu \\
n
\end{array}\right)(-1)^{n}\left\{\int_{0}^{2 \pi} \mathrm{d} \alpha \mathrm{e}^{i K^{\prime} \alpha} \sin ^{n} \alpha \cos ^{\mu-n} \alpha\right\}\right. \\
& \left.\times\left\{\int_{0}^{2 \pi} \mathrm{d} \gamma \mathrm{e}^{i K^{\gamma}} \sin ^{n} \gamma \cos ^{\mu-n} \gamma\right\}\left\{\int_{-1}^{1} \mathrm{~d} \cos \beta \cos ^{\lambda+n} \beta \mathrm{d}_{K^{\prime} K}^{L}(\beta)\right\}\right] .
\end{aligned}
$$

The phase in the determination of $a(K, L)$ was chosen such that

$$
a(K, L)>0 \text { for } K \geqq 0, \quad|(\lambda \mu) K L M\rangle=|(\lambda \mu)-K L M\rangle
$$

(when the representation must be denoted explicitly, we shall write $a((\lambda \mu) K L)$.

In cases when $\lambda<\mu$, it is more convenient to project the angular momentum states out of the lowest-weight state, i.e. the state corresponding to $p=\lambda, q=\mu$, $r=\lambda$. Such states will be denoted by a $\bar{K}$.

$$
|(\lambda \mu) \bar{K} L M\rangle=P\left|(\dot{\lambda} \mu) \varepsilon_{L} \Lambda_{L} \Lambda_{0 L}\right\rangle
$$

The two types of states are related by

$$
|(\lambda \mu) \bar{K} L M\rangle=\sum_{K_{1}} \frac{a\left(K_{1}, L\right)}{a(K, L)}\left\langle L \bar{K} \mid F(\hat{\lambda}, \mu, \hat{\lambda}) i L K_{1}\right\rangle\left|(\hat{\lambda} \mu) K_{1} L M\right\rangle .
$$

The above summation runs over both negative and positive allowed values of $K_{1}$. Use was made of the fact that $a(\bar{K}, L)=a(K, L)$. The operator $F(\lambda, \mu, \lambda)$ is such that it constructs the lowest-weight state when operating on the highest weight state, i.e.

$$
\left|(\lambda \mu) \varepsilon_{L} \Lambda_{L} \Lambda_{0 L}\right\rangle=F(\lambda, \mu, \lambda)\left|(\lambda \mu) \varepsilon_{H} \Lambda_{H} \Lambda_{0 H}\right\rangle
$$

The operator $F(p, q, r)$ defined in eq. (2) can be expressed as a function of angular momentum operators alone ${ }^{2}$ ). 


\section{Phase convention and orthogonalization of the basis}

Elliott's phase convention has the disadvantage that some of the $S U(3) \supset R(3)$ Wigner coefficients become pure imaginary if one is determined to maintain the wellestablished, Condon-Shortly ${ }^{19}$ ) convention of the $\mathrm{R}(3)$ Wigner coefficients and the phase conventions of the $S U(3) \supset S U(2)$ Wigner coefficients ${ }^{3}$ ). With the phase convention

$$
|(\lambda \mu) K L M\rangle_{0}=i^{\lambda+2 \mu}|(\lambda \mu) K L M\rangle,
$$

it is possible to make all the $S U(3) \supset R(3)$ Wigner coefficients real. The new phase convention also implies

$$
|(\lambda \mu) \bar{K} L M\rangle_{0}=i^{2 \lambda+3 \mu}|(\lambda \mu) \bar{K} L M\rangle .
$$

In the present work an orthonormal basis, to be denoted by $|(\lambda \mu) \kappa L M\rangle$, is constructed which is tied as closely as possible to Elliott's basis and is obtained from it through the Gram-Schmidt ${ }^{17}$ ) orthogonalization process according to a systematic recipe. Let $K_{1}, K_{2}, \ldots K_{n}$ be the Elliott rotational bands ${ }^{1,2}$ ) that occur in a given representation with $K_{1}<K_{2}<\ldots<K_{n}$. The new basis is then defined by

$$
\begin{aligned}
& \left.\left|(\lambda \mu) \kappa_{1} L M\right\rangle=\mathrm{j}(\lambda \mu) K_{1} L M\right\rangle_{0}, \\
& \left|(\lambda \mu) \kappa_{2} L M\right\rangle=X_{21}\left|(\lambda \mu) K_{1} L M\right\rangle_{0}+X_{22}\left|(\lambda \mu) K_{2} L M\right\rangle_{0}, \\
& \left|(\lambda \mu) \kappa_{i} L M\right\rangle=\sum_{j=1}^{i} X_{i j}\left|(\lambda \mu) K_{j} L M\right\rangle_{0} .
\end{aligned}
$$

The coefficients $X_{i j}$ are determined by the requirement

$$
\left\langle(\hat{\lambda} \mu) \kappa_{i} L M \mid(\lambda \mu) \kappa_{j} L M\right\rangle=\delta_{i j} .
$$

The new basis has the useful property that the $\kappa$-states for fixed $\mu$, given $L, M$, go over to the corresponding states of Elliott (with the modified phase) in the limit

$$
\lim _{\lambda \rightarrow \infty}|(\lambda \mu) \kappa L M\rangle=|(\lambda \mu) K L M\rangle_{0} .
$$

This relation was used to fix the overall phase of the transformation coefficients $X_{i j}$.

In the above notation, the (84) representation, for example, has $K=0,2,4$. The orthonormal set of basis vectors will be $|(84) \kappa L M\rangle$ with $\kappa=0,2,4$. The states $\kappa=0$ are the states of the lowest, or $K=0$, Elliott rotational band. The states with $\kappa=2$ are a mixture of $K=0$ and $K=2$ Elliott states orthogonal to $\kappa=0$. The $\kappa=4$ states are mixture of $K=0, K=2$ and $K=4$ states which are orthogonal to both $\kappa=0$ and $\kappa=2$ states. The coefficients $X_{i j}$ are explicitly given in table $2 \mathrm{~B}$ for the cases $(\lambda 2)$ and $(\lambda 3)$. We also give them for $(\lambda 4)$ in the case when $\lambda-L=$ odd, i.e. when the multiplicity of $L$ is 2 .

We emphasize here that $K$ and, consequently $\kappa$, is assigned according to $\mu$. Hence the possible values for $K$ (or $\kappa$ ) are $\mu, \mu-2, \mu-4, \ldots 1$ or 0 . From the definition of $\kappa$, it is clear that if a given $L$ occurs in a given representation only once, it belongs to the 
TABLE 2A

Elliott's $a((\lambda \mu) K L)$ coefficients in algebraic form for arbitrary $\lambda$

\begin{tabular}{|c|c|c|c|}
\hline$\mu$ & $\boldsymbol{K}$ & $\lambda-L=$ even & $\lambda-L=$ odd \\
\hline$\mu=0$ & 0 & {$[(2 L+1) b(\lambda, L)]^{\frac{1}{2}}$} & \\
\hline$\mu=1$ & 1 & {$\left[\frac{2 L+1}{4} b(\lambda, L)\right]^{\frac{1}{2}}$} & {$\left[\frac{(2 L+1)(\lambda+2)}{4(\lambda+1)} b(\lambda+1, L)\right]^{\frac{1}{2}}$} \\
\hline \multirow{2}{*}{$\mu=2-$} & 0 & {$\left[\frac{(2 L+1) \varphi(\lambda+1, L)}{4(\lambda+1)(\lambda+2)} b(\lambda+2, L)\right]^{\frac{1}{2}}$} & \\
\hline & 2 & {$\left[\frac{(2 L+1) \varphi(\lambda+2, L)}{16(\lambda+1)(\lambda+2)} b(\lambda+2, L)\right]^{\frac{1}{2}}$} & {$\left[\frac{(2 L+1)(\lambda+3)}{8(\lambda+1)} b(\lambda+1, L)\right]^{\frac{1}{2}}$} \\
\hline \multirow{2}{*}{$\mu=3$} & 1 & {$\left[\frac{3(2 L+1) W(\lambda+1, L)}{64(\lambda+1)(\lambda+2)} b(\lambda+2, L)\right]^{\frac{1}{2}}$} & {$\left[\frac{3(2 L+1)(\lambda+4) \chi(\lambda+1, L)}{64(\lambda+1)(\lambda+2)(\lambda+3)} b(\lambda+3, L)\right]^{\frac{1}{2}}$} \\
\hline & 3 & {$\left[\frac{(2 L+1) \chi(\lambda+3, L)}{64(\lambda+1)(\lambda+2)} b(\lambda+2, L)\right]^{\frac{1}{2}}$} & {$\left[\frac{(2 L+1) W(\lambda+3, L)(\lambda+4)}{64(\lambda+1)(\lambda+2)(\lambda+3)} b(\lambda+3, L)\right]^{\frac{1}{t}}$} \\
\hline \multirow{3}{*}{$\mu=4$} & 0 & {$\left[\frac{3(2 L+1) P(\lambda, L)}{64(\lambda+1)(\lambda+2)(\lambda+3)(\lambda+4)} b(\lambda+4, L)\right]$} & $\frac{1}{2}$ \\
\hline & 2 & {$\left[\frac{(2 L+1) Q(\lambda, L)}{16(\lambda+1)(\lambda+2)(\lambda+3)(\lambda+4)} b(\lambda+4, L)\right]^{\frac{1}{2}}$} & {$\left[\frac{(2 L+1)(\lambda+5) Z(\lambda+1, L)}{16(\lambda+1)(\lambda+2)(\lambda+3)} b(\lambda+3, L)\right]^{\frac{1}{2}}$} \\
\hline & 4 & {$\left[\frac{(2 L+1) R(\lambda, L)}{2^{12}(\lambda+1)(\lambda+2)(\lambda+3)(\lambda+4)} b(\lambda+4, L)\right]^{\frac{1}{2}}$} & {$\left[\frac{(2 L+1)(\lambda+5) Z(\lambda+4, L)}{64(\lambda+1)(\lambda+2)(\lambda+3)} b(\lambda+3, L)\right]^{\frac{1}{2}}$} \\
\hline
\end{tabular}

where

$$
\begin{aligned}
b(\lambda, L)= & \frac{\lambda(\lambda-1)(\lambda-2) \ldots(\lambda-L+1)}{(\lambda-L+1)(\lambda-L+3) \ldots(\lambda+L-1)(\lambda+L+1)}, \quad L \geqq 1 \\
b(\lambda, 0)= & \frac{1}{\lambda+1}, \\
\varphi(\lambda, L)= & 2(\lambda+1)^{2}-L(L+1), \quad \chi(\lambda, L)=4(\lambda+1)^{2}-(L-1)(L+2) \\
W(\lambda, L)= & 4 \lambda(\lambda+2)-3(L-1)(L+2), \quad Z(\lambda, L)=2 \lambda(\lambda+2)-(L-2)(L+3) \\
P(\lambda, L)= & 3(\lambda-L+4)(\lambda-L+2)(\lambda+L+3)(\lambda+L+5)+2(\lambda+1)(\lambda+2)(\lambda-L+4)(\lambda+L+5) \\
& +3(\lambda+1)(\lambda+2)(\lambda+3)(\lambda+4), \\
Q(\lambda, L)= & 2(\lambda+5)^{2}\left(\lambda^{2}+4 \lambda+6\right)-2\left(\lambda^{2}+7 \lambda+16\right) L-\left(2 \lambda^{2}+14 \lambda+31\right) L^{2}+2 L^{3}+L^{4}, \\
R(\lambda, L)= & (\lambda-L+11)(\lambda-L+9)(\lambda-L+7)(\lambda-L+5)+28(\lambda-L+9)(\lambda-L+7)(\lambda-L+5)(\lambda+L+5) \\
+ & 70(\lambda-L+7)(\lambda-L+5)(\lambda+L+3)(\lambda+L+5)+28(\lambda-L+5)(\lambda+L+1)(\lambda+L+3)(\lambda+L+5) \\
+ & (\lambda+L-1)(\lambda+L+1)(\lambda+L+3)(\lambda+L+5)
\end{aligned}
$$


TABLE 2B

Coefficients $X_{\kappa K}$ for the expansion $\left|(\lambda \mu)_{\kappa} L M\right\rangle=\Sigma X_{\kappa K}|(\lambda \mu) K L M\rangle$

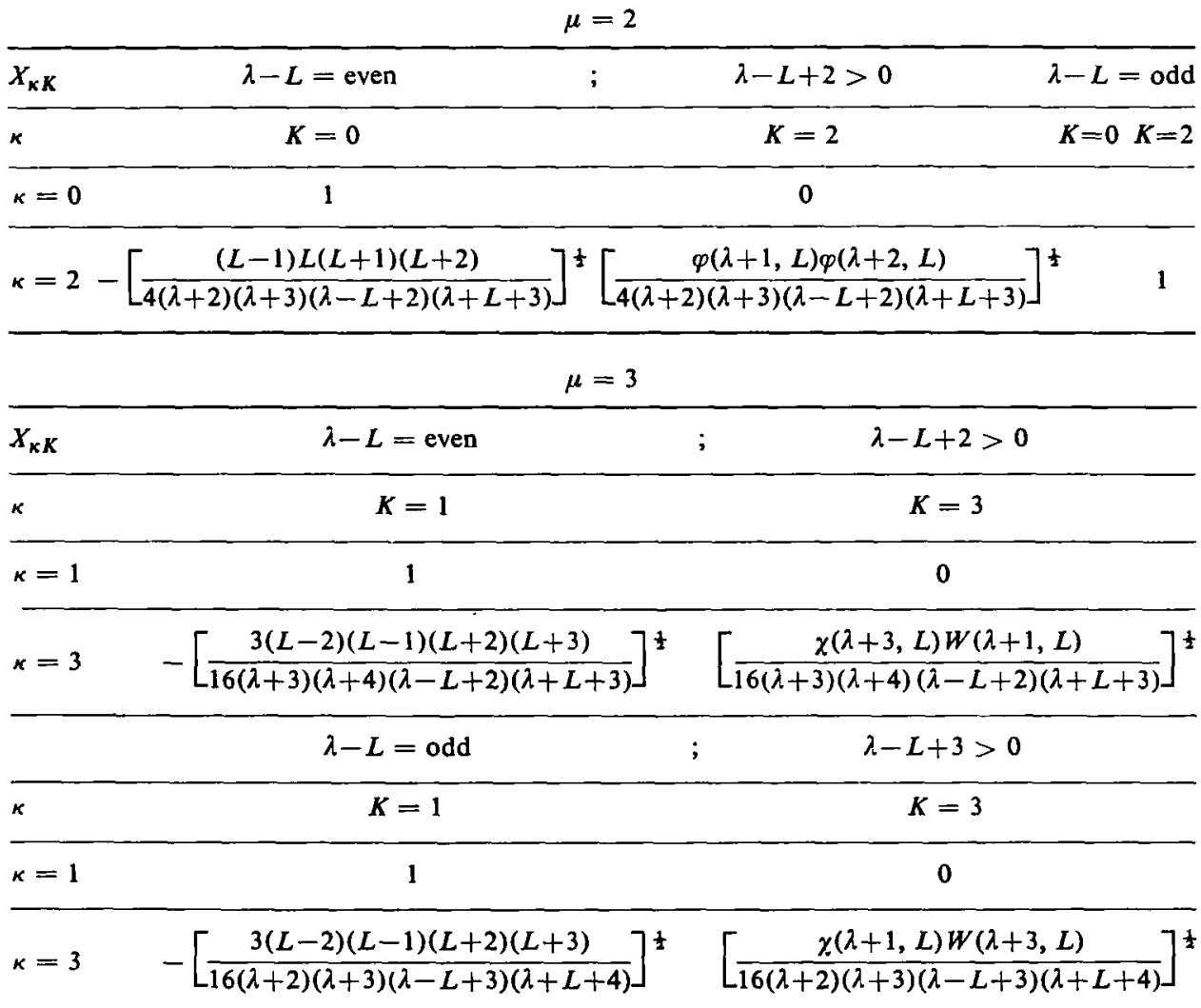

where

$\varphi(\lambda, L)=2(\lambda+1)^{2}-L(L+1)$,

$\chi(\lambda, L)=4(\lambda+1)^{2}-(L-1)(L+2)$,

$W(\lambda, L)=4 \lambda(\lambda+2)-3(L-1)(L+2)$.

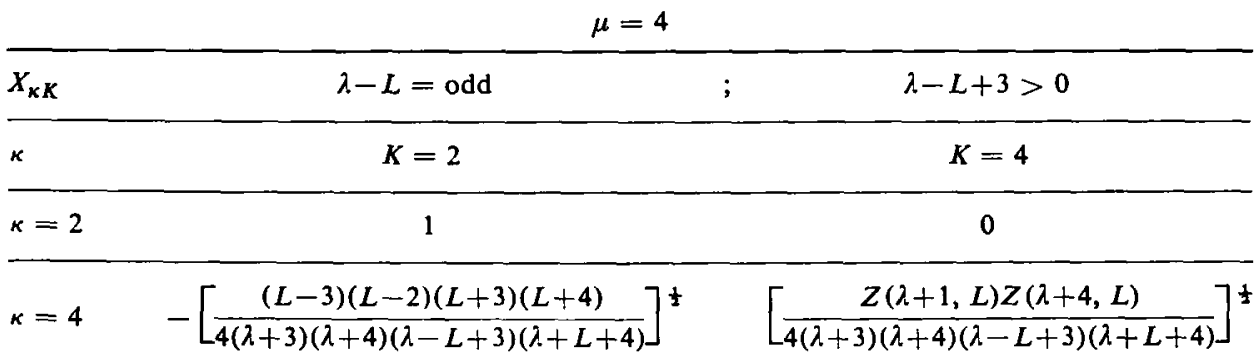

where

$Z(\dot{\lambda}, L)=2 \lambda(\lambda+2)-(L-2)(L+3)$. 
lowest possible $\kappa$-band. If it occurs twice, it belongs to the two lowest possible $\kappa$-bands. If it occurs three times, it belongs to the three lowest possible $\kappa$-bands, etc. The only exception occurs when $\kappa=0$. The allowed $L$-values are restricted to be even or odd for $\lambda$ even or odd, respectively; e.g. for (84) we have

$\kappa=0$ $L=0,2,4,6,8,10,12$,

$\kappa=2$ $L=2,3,4,5,6,7,8,9,10,11$,

$\kappa=4$ $L=4,5,6,7,8,9$,

for (93) we have

$\kappa=1$ $L=1,2,3,4,5,6,7,8,9,10,11,12$,

$\kappa=3$

$L=3,4,5,6,7,8,9,10$.

Exactly in an analogous way we orthogonalize the states $|(\lambda \mu) \bar{K} L M\rangle$. The orthogonalized states are now denoted by $|(\lambda \mu) \bar{\kappa} L M\rangle$ (both $\bar{K}$ and $\bar{\kappa}$ are now assigned according to $\lambda$ ); e.g., in (37) we have
$\bar{\kappa}=1$
$L=1,2,3,4,5,6,7,8,9,10$,
$\bar{\kappa}=3$
$L=$
$3,4,5,6,7,8$.

With the above phase conventions it can be shown that

$$
|(\lambda \mu) \kappa L M\rangle^{*}=(-1)^{\lambda}(-1)^{L-M}|(\mu \lambda) \bar{\kappa} L-M\rangle .
$$

For the self-contragredient representations, $\lambda=\mu$, we have

$$
|(\hat{\lambda} \lambda) \bar{\kappa} L M\rangle=\sum_{\kappa} C(\lambda, \kappa, L)|(\lambda \lambda) \kappa L M\rangle
$$

the cases $(\lambda \mu)=(11)$ or (22) are of particular interest. For them

$$
\begin{aligned}
& |(11) \bar{\kappa}=1 L M\rangle=(-1)^{L+1}|(11) \kappa=1 L M\rangle, \\
& |(22) \bar{\kappa}=02 M\rangle=-\frac{1}{13}\{8|(22) \kappa=02 M\rangle+\sqrt{105}|(22) \kappa=22 M\rangle\}, \\
& |(22) \bar{\kappa}=22 M\rangle=-\frac{1}{13}\{\sqrt{105}|(22) \kappa=02 M\rangle-8|(22) \kappa=22 M\rangle\}, \\
& |(22) \bar{\kappa}=04 M\rangle=|(22) \kappa=04 M\rangle ;|(22) \bar{\kappa}=000\rangle=|(22) \kappa=000\rangle, \\
& |(22) \bar{\kappa}=23 M\rangle=-|(22) \kappa=23 M\rangle .
\end{aligned}
$$

Also

where

$$
\begin{aligned}
|(0 \hat{\lambda}) \bar{\kappa} L M\rangle & =(-1)^{\frac{1}{2}(\lambda-L)}|(0 \hat{\lambda}) \kappa L M\rangle, \\
|(\lambda 0) \bar{\kappa} L M\rangle & =(-1)^{\lambda}(-1)^{\frac{1}{2}(\lambda-L)}|(\lambda 0) \kappa L M\rangle \\
|(1 \lambda) \bar{\kappa} L M\rangle & =(-1)^{\lambda-\alpha}|(1 \hat{\lambda}) \kappa L M\rangle, \\
|(\lambda 1) \bar{\kappa} L M\rangle & =(-1)^{1-\alpha}|(\lambda 1) \kappa L M\rangle,
\end{aligned}
$$

$$
\alpha=\frac{1}{2}[\lambda+L+1] \text { with }[x]=\text { integral part of } x \text {. }
$$


Since the EHiott states themselves may be of some importance in some applications, we give the above expresisons in the non-orthogonal basis as well

$$
\begin{aligned}
& |(11) \bar{K}=1 L M\rangle=(-1)^{L}|(11) K=1 L M\rangle, \\
& |(22) \bar{K}=02 M\rangle=-\frac{1}{2}\left\{|(22) K=02 M\rangle+\sqrt{\frac{33}{13}}|(22) K=22 M\rangle\right\}, \\
& |(22) \bar{K}=22 M\rangle=\frac{1}{2}\left\{-\sqrt{\frac{39}{11}}|(22) K=02 M\rangle+|(22) K=22 M\rangle\right\}, \\
& |(22) \bar{K}=04 M\rangle=\frac{1}{4}\{19|(22) K=04 M\rangle-15|(22) K=24 M\rangle\}, \\
& |(22) \bar{K}=24 M\rangle=-3|(22) K=04 M\rangle+4|(22) K=24 M\rangle, \\
& |(22) \bar{K}=23 M\rangle=-|(22) K=23 M\rangle ;|(22) \bar{K}=000\rangle=|(22) K=000\rangle .
\end{aligned}
$$

\section{4. $\mathrm{SU}(3) \supset \mathbf{R}(3)$ Wigner coefficients}

After an orthonormal basis has been introduced we can discuss the unitary transformation that leads us from the product space spanned by the basis $\left|\left(\lambda_{1} \mu_{1}\right) \kappa_{1} L_{1} M_{1}\right\rangle \mid$ $\left|\left(\lambda_{2} \mu_{2}\right) \kappa_{2} L_{2} M_{2}\right\rangle$ to the space spanned by the coupled vectors $\mid\left(\lambda_{1} \mu_{1}\right)\left(\lambda_{2} \mu_{2}\right)$; $\left(\lambda_{3} \mu_{3}\right) \rho \kappa_{3} L_{3} M_{3}>$ whose coefficients are the SU(3) Wigner coefficients, i.e.

$$
\begin{aligned}
& \left|\left(\lambda_{1} \mu_{1}\right),\left(\lambda_{2} \mu_{2}\right) ;\left(\lambda_{3} \mu_{3}\right) \rho \kappa_{3} L_{3} M_{3}\right\rangle \\
& =\sum_{\substack{\kappa_{1} L_{1} M_{1} \\
\kappa_{2} L_{2}\left(M_{2}\right)}}\left\langle\left(\lambda_{1} \mu_{1}\right) \kappa_{1} L_{1} M_{1} ;\left(\lambda_{2} \mu_{2}\right) \kappa_{2} L_{2} M_{2}\right|\left(\lambda_{3} \mu_{3} \kappa_{3} L_{3} M_{3}\right\rangle_{\rho} \\
& \quad \times\left|\left(\lambda_{1} \mu_{1}\right) \kappa_{1} L_{1} M_{1}\right\rangle\left|\left(\lambda_{2} \mu_{2}\right) \kappa_{2} L_{2} M_{2}\right\rangle
\end{aligned}
$$

subject to the familiar angular momentum restrictions on the indices. The quantum number $\rho$ [refs. $\left.{ }^{3,11}\right)$ ] is necessary because the product is not in general simply reducible, i.e. a representation $\left(\lambda_{3} \mu_{3}\right)$ may occur more than once. The choice of $\rho$ in the present work is exactly the one adopted by Hecht ${ }^{3}$ ).

In a completely analogous fashion as in the intrinsic basis, the $M$-dependence can be factored out ${ }^{3,13}$ ), i.e.

$$
\begin{aligned}
& \left\langle\left(\lambda_{1} \mu_{1}\right) \kappa_{1} \kappa_{1} M_{1} ;\left(\lambda_{2} \mu_{2}\right) \kappa_{2} L_{2} M_{2} \mid\left(\lambda_{3} \mu_{3}\right) \kappa_{3} L_{3} M_{3}\right\rangle_{\rho}=\left\langle\left(\lambda_{1} \mu_{1}\right) \kappa_{1} L_{1} ;\right. \\
& \left.\left(\lambda_{2} \mu_{2}\right) \kappa_{2} L_{2}||\left(\lambda_{3} \mu_{3}\right) \kappa_{3} L_{3}\right\rangle_{\rho}\left\langle L_{1} M_{1} ; L_{2} M_{2} \mid L_{3} M_{3}\right\rangle .
\end{aligned}
$$

From the unitarity of the full SU(3) and the ordinary R(3) Wigner coefficients, it follows that the "double-bar" coefficients are also unitary. With the phase convention introduced above they become real, i.e. orthogonal. As in the intrinsic scheme it can be shown ${ }^{3}$ ) that

$$
\begin{aligned}
& \sum_{\rho_{1,23}}\left\langle\left(\lambda_{1} \mu_{1}\right) \kappa_{1} L_{1} ;\left(\lambda_{23} \mu_{23}\right) \kappa_{23} L_{23} \|\right. \\
& \quad \times(\lambda \mu) \kappa L\rangle_{\rho_{1,23}} U\left(\left(\lambda_{1} \mu_{1}\right)\left(\lambda_{2} \mu_{2}\right)(\lambda \mu)\left(\lambda_{3} \mu_{3}\right) ;\right. \\
& \quad \times\left(\lambda_{12} \mu_{12}\right) \rho_{12} \rho_{12,3}\left(\left(\lambda_{23} \mu_{23}\right) \rho_{23} \rho_{1,23}\right)=\sum_{\substack{\kappa_{3} \kappa_{2} L_{2} L_{3} \\
\kappa_{12} L_{12}}}\left\langle\kappa_{1} L_{1} ; \kappa_{2} L_{2} \mid\left(\lambda_{12} \mu_{12}\right) \kappa_{12} L_{12}\right\rangle_{\rho_{12}} \\
& \quad \times\left\langle\kappa_{2} L_{2} ; \kappa_{3} L_{3} \|\left(\lambda_{23} \mu_{23}\right) \kappa_{23} L_{23}\right\rangle_{\rho_{23}}\left\langle\kappa_{12} L_{12} ; \kappa_{3} L_{3} \mid(\lambda \mu) \kappa L\right\rangle_{\rho_{123},} U\left(L_{1} L_{2} L L_{3} ;\right. \\
& \left.L_{12} L_{23}\right)
\end{aligned}
$$


The $U$-functions depend only on the representations involved in the coupling and not on the specific subgroup chosen to specify the states. Most of the SU(3) $U$-functions needed for $2 \mathrm{~s}-1 \mathrm{~d}$ shell calculations have been calculated $\left.{ }^{3,18}\right)$. A few additional $U$ functions are needed, in particular those with $\left(\lambda_{2} \mu_{2}\right)=(10)$ or $(01)$ and $\left(\lambda_{3} \mu_{3}\right)=(10)$. They have been calculated and are given in table 1 .

Eq. (14) is basic in the present work, because it is used in a build-up process to construct more complicated Wigner coefficients starting from very simple ones by proper choice of the representations $\left(\lambda_{2} \mu_{2}\right),\left(\lambda_{3} \mu_{3}\right)$ and $\left(\lambda_{12} \mu_{12}\right)$. Since we can start from simple coefficients on the right-hand side of eq. (14), which do not involve labels $\rho$, the equation serves as a definition of $\rho_{1,23}$ in $\left\langle\left(\lambda_{1} \mu_{1}\right) \kappa_{1} L_{1} ;\left(\lambda_{23} \mu_{23}\right) \kappa_{23} L_{23}\right|$ $|(\lambda \mu) L\rangle_{\rho_{1,23}}$ if we pick the corresponding SU(3) $U$-functions as defined by Hecht ${ }^{3}$ ).

It turns out that all $\mathrm{SU}(3) \supset \mathrm{R}(3)$ Wigner coefficients can be constructed via the above "building-up" process if the SU(3) $U$-functions and all coefficients $\left\langle\left(\lambda_{1} \mu_{1}\right) \kappa_{2} L_{2} ;(10) 1 \|\left(\lambda_{3} \mu_{3}\right) \kappa_{3} L_{3}\right\rangle$ are available.

\section{Coefficients for the product $\left(\lambda_{1} \mu_{1}\right) \times(10) \rightarrow\left(\lambda_{3} \mu_{3}\right)$}

Engeland ${ }^{1}$ ) has shown that the coefficients in the expansion of a coupled wave function $|(\lambda \mu) K L M\rangle$ in terms of the individual wave functions $\left|\left(\lambda_{1} \mu_{1}\right) K_{1} L_{1} M_{1}\right\rangle$ $\left|\left(\lambda_{2} \mu_{2}\right) K_{2} L_{2} M_{2}\right\rangle$ in Elliott's basis, namely ${ }^{\dagger}$

$$
\begin{array}{r}
|(\lambda \mu) K L M\rangle=\sum_{\substack{K_{1} L_{1} M_{1} \\
K_{2} L_{2}\left(M_{2}\right)}} C\left(\left(\lambda_{1} \mu_{1}\right) K_{1} L_{1},\left(\lambda_{2} \mu_{2}\right) K_{2} L_{2} ;(\lambda \mu) K L\right)\left\langle L_{1} M_{1} L_{2} M_{2} \mid L M\right\rangle \\
\times\left|\left(\lambda_{1} \mu_{1}\right) K_{1} L_{1} M_{1}\right\rangle\left|\left(\lambda_{2} \mu_{2}\right) K_{2} L_{2} M_{2}\right\rangle
\end{array}
$$

are given by the expression

$$
\begin{gathered}
C\left(\left(\lambda_{1} \mu_{1}\right) K_{1} L_{1},\left(\lambda_{2} \mu_{2}\right) K_{2} L_{2} ;(\lambda \mu) K L\right\rangle=\frac{a\left(\left(\lambda_{1} \mu_{1}\right) K_{1} L_{1}\right) a\left(\left(\lambda_{2} \mu_{2}\right) K_{2} L_{2}\right)}{a((\lambda \mu) K L)} \\
\times \sum_{\substack{\varepsilon_{1}, \lambda_{1}, \lambda_{01} \\
\varepsilon_{2} \lambda_{2} \lambda_{02}}}\left\langle\left(\lambda_{1} \mu_{1}\right) \varepsilon_{1} \Lambda_{1} ;\left(\lambda_{2} \mu_{2}\right) \varepsilon_{2} \Lambda_{2}||(\lambda \mu) \varepsilon_{H} \Lambda_{H}\right\rangle\left\langle\Lambda_{1} \Lambda_{01} \Lambda_{2} \Lambda_{02} \mid \Lambda_{H} \Lambda_{0 H}\right\rangle \\
\times \sum_{K_{1}^{\prime}, K^{\prime}{ }_{2}}\left\langle L_{1} K_{1}^{\prime}\left|F_{1}\right| L_{1} K_{1}\right\rangle\left\langle L_{2} K_{2}^{\prime}\left|F_{2}\right| L_{2} K_{2}\right\rangle\left\langle L_{1} K_{1}^{\prime} L_{2} K_{2}^{\prime} \mid L K\right\rangle .
\end{gathered}
$$

The summations in eqs. (15) and (16) run over both positive and negative allowed $K$-values. The operator $F_{i} i=1,2$ is defined by

$$
\left|\left(\lambda_{i} \mu_{i}\right) \varepsilon_{i} \Lambda_{i} \Lambda_{0 i}\right\rangle=F_{i}\left|\left(\lambda_{i} \mu_{i}\right) \varepsilon_{i H} \Lambda_{i H} \Lambda_{0 i H}\right\rangle, \quad i=1,2 .
$$

There are some difficulties in a direct application of the above formula. First, although the operators $F_{i}$ can be expressed as functions of the angular momentum operators alone,

+ Engeland uses the notation $\left\langle\left(\lambda_{1} \mu_{1}\right) K_{1} L_{1},\left(\lambda_{2} \mu_{2}\right) K_{2} L_{2} \mid(\lambda \mu) K L\right\rangle$ for the expansion coefficients $C\left(\left(\lambda_{1} \mu_{1}\left(K_{1} L_{1},\left(\lambda_{2} \mu_{2}\right) K_{2} L_{2} ;(\lambda \mu) K L\right)\right.\right.$. Such notation has purposely been avoided here, since these coefficients do not constitute a Gram matrix, i.e. they do not represent the scalar product connecting the $|(\lambda \mu) K L M\rangle$ basis to the $\left|\left(\lambda_{1} \mu_{1}\right) K_{1} L_{1} M_{1}\right\rangle\left|\left(\lambda_{2} \mu_{2}\right) K_{2} L_{2} M_{2}\right\rangle$ basis. They are not yet $\mathrm{SU}(3) \supset \mathrm{R}(3)$ Wigner coefficients. 
it is very difficult to give the explicit algebraic forms of these functions in the general case. However, if $\left(\lambda_{2} \mu_{2}\right)=(10)$, this can be accomplished since the possible $F_{i}$ are restricted to those needed to construct states removed by only a few steps from the highest-weight states. Second, after the integrations have been performed, the formula for $a(K, L)$ is too complicated to be useful for algebraic expressions. However, if $\left(\lambda_{2} \mu_{2}\right)=(10)$ we show in the appendix that we can write the coefficients $a(K, L)$ such that a simple algebraic expression can be given for the ratio $a\left(\left(\lambda_{1} \mu_{1}\right) K_{1} L_{1}\right) /(a(\lambda \mu) K L)$. But one of the difficulties inherent in the use of a basis in which $L^{2}$ and $L_{0}$ are diagonal is the fact that the $a(K, L)$ become more and more complicated as the multiplicity of $L$ increases, e.g. in the case of the representation ( $\lambda 4)$ when the multiplicity of $L$ is three, $a((\lambda 4) K L)$ contains a factor which is a rather complicated irreducible polynomial of fourth degree in $\lambda$ and $L$. Since this factor is carried through the calculation, it not only makes the algebra tedious but sets a limit as to how far one can go in deriving useful algebraic expressions. An extra complication is associated with the fact that the coefficients $a((\lambda \mu) K L)$ for a given $\mu$ have a different form depending on whether the difference $\lambda-L$ is even or odd. Expressions for $a((\lambda \mu) K L)$ are given in table $2 \mathrm{~A}$.

By making the transition from the basis $\left|\left(\lambda_{i} \mu_{i}\right) K_{i} L_{i} M_{i}\right\rangle$ to the orthogonal basis $\left|\left(\lambda_{i} \mu_{i}\right) \kappa_{i} L_{i} M_{i}\right\rangle$ defined in sect. 3, Engeland's expansion coefficients $C$ can be transformed into $\mathrm{SU}(3) \supset \mathrm{R}(3)$ Wigner coefficients. The specific relation between the two sets of coefficients is given by

$$
\begin{aligned}
\left\langle\left(\lambda_{1} \mu_{1}\right) \kappa_{\alpha_{1}} L_{1} ;\left(\lambda_{1} \mu_{2}\right) \kappa_{\alpha_{2}} L_{2} \|\left(\lambda_{3} \mu_{3}\right) \kappa_{\alpha_{3}} L_{3}\right\rangle \\
=i^{\left(\lambda_{3}-\lambda_{1}-\lambda_{2}+2\left(\mu_{3}-\mu_{1}-\mu_{2}\right)\right)} \sum_{\substack{i_{1} i_{2} i_{3} \\
i^{\prime} i^{\prime}{ }_{2}}} X_{\alpha_{1}, i_{1}} X_{\alpha_{2}, i_{2}} X_{\alpha_{3}, i_{3}} \\
\quad \times\left\langle\left(\lambda_{1} \mu_{1}\right) K_{i_{1}} L_{1} M_{1} \mid\left(\lambda_{1} \mu_{1}\right) K_{i^{\prime} 1} L_{1} M_{1}\right\rangle\left\langle\left(\lambda_{2} \mu_{2}\right) K_{i_{2}} L_{2} M_{2} \mid\left(\lambda_{2} \mu_{2}\right) K_{i^{\prime}{ }_{2}} L_{2} M_{2}\right\rangle \\
\quad \times C\left(\left(\lambda_{1} \mu_{1}\right) K_{i^{\prime}{ }_{1}} L_{1},\left(\lambda_{2} \mu_{2}\right) K_{i^{\prime}{ }_{2}} L_{2} ;\left(\lambda_{3} \mu_{3}\right) K_{i_{3}} L_{3}\right) .
\end{aligned}
$$

The above formidable formula for $\left(\lambda_{2} \mu_{2}\right)=(10)$ becomes

$$
\begin{aligned}
& \left\langle\left(\lambda_{1} \mu_{1}\right) \kappa_{\alpha_{1}} L_{1} ;(10) 1 \|\left(\lambda_{3} \mu_{3}\right) \kappa_{\alpha_{3}} L_{3}\right\rangle=i^{\left(\lambda_{3}+2 \mu_{3}-\lambda_{1}-2 \mu_{1}-1\right)} \sum_{i_{1}, i_{2}, i^{\prime} 1} X_{\alpha_{1}, i_{1}} X_{\alpha_{3}, i_{3}} \\
& \quad \times\left\langle\left(\lambda_{1} \mu_{1}\right) K_{i_{1}} L_{1} M_{1} \mid\left(\lambda_{1} \mu_{1}\right) K_{i^{\prime}{ }_{1}} L_{1} M_{1}\right\rangle C\left(\left(\lambda_{1} \mu_{1}\right) K_{i^{\prime} 1},(10) 1 ;\left(\lambda_{3} \mu_{3}\right) K_{i_{3}} L_{3}\right) .
\end{aligned}
$$

The coefficients $X_{i j}$ as defined by eq. (10) are functions of the overlaps between the different possible Elliott bands. In actually applying eq. (17b), one encounters difficulties only when $\mu_{3}>\mu_{1}$. Such difficulties can be overcome most easily by a systematic process of calculation starting from the smallest possible $\mu_{1}$. When $\mu_{1}=0$, Engeland's coefficients are already elements of an orthogonal matrix. When $\mu_{1}=0$, Engeland's matrix is non-orthogonal, but nevertheless it is a Gram matrix. Therefore the overlaps $\left\langle K_{i_{1}} \mid K_{i_{1}{ }_{1}}\right\rangle$ and $X_{\alpha_{1}, i_{1}}$ do not appear in eq. (17b). The coefficient $X_{\alpha_{3}, i_{3}}$ appears only in the case $\mu_{3}=2$ and can be determined by the requirement that $\left\langle\left(\lambda_{1} \mu_{1}\right) \kappa_{\alpha_{1}} L_{i} ;(10) 1 \|\left(\lambda_{3} \mu_{3}\right) \kappa_{\alpha_{3}} L_{3}\right)$ be orthogonal or equivalently by eq. (10b). Then 
one proceeds to the next step of the calculation, $\left(\lambda_{1} \mu_{1}\right)=(\lambda 2)$. All the factors on the right hand side of eq. (17b) are known except $X_{\alpha_{3}, i_{3}}$ when $\mu_{3}=3$, which is determined in exactly the same way as before, etc. The phase convention introduced by eqs. (9a) and (9b) makes the $S U(3) \supset R(3)$ Wigner coefficients real.

Because of the complications mentioned above, however, it is not possible to give general algebraic expressions for the Wigner coefficients $\left\langle\left(\lambda_{1} \mu_{1}\right) \kappa_{1} L_{1} ;(10) 1 \|\left(\lambda_{3} \mu_{3}\right)\right.$ $\kappa_{3} L_{3}>$ for arbitrarily large values of the SU(3) quantum numbers. If the representations $\left(\lambda_{1} \mu_{1}\right),\left(\lambda_{3} \mu_{3}\right)$ are restricted to these in which the multiplicity of a specific $L$ value is at most two, the algebraic form of the Wigner coefficients is simple enough to be useful. The coefficients involving the representations $\left(\lambda_{1} \mu_{1}\right),\left(\lambda_{3} \mu_{3}\right)$ with $\mu_{1}$, $\mu_{3}<3$ are tabulated in tables 3-5. Since representations with $\mu=4$ are important for $2 \mathrm{~s}-1 \mathrm{~d}$ shell calculations, a sufficient number of intermediate calculational steps are given so that these coefficients can also be calculated, although their algebraic form for general $\lambda$ and $L$ is too complicated to be very useful.

Since the states $|(\lambda 4) \kappa L M\rangle$ with $\lambda-L=$ odd have $L$-multiplicity of at most two, Wigner coefficients involving only these ( $\lambda 4)$ states are simple. Their general form is included in tables 5 . The remaining generating coefficients involving the representation ( 24$)$ are of three types: (i) the coefficients $\left\langle(\lambda 3) \kappa_{1} L_{1} ;(10) 1 \|(\lambda-1,4) \kappa L\right\rangle$ with $\lambda-L$ odd, (ii) $\left.\left\langle(\lambda 4) \kappa_{1} L_{1} ;(10) 1\right|(\lambda 3) \kappa L\right)$ with $\lambda-L_{1}=$ even and (iii) $\left\langle(\lambda 4) \kappa_{1} L_{1}\right.$; (10) $1 \|(\lambda+1,4) \kappa L\rangle$ for any $L_{1}, L$. In case of the coefficients (i), it is feasible to give the coefficients $\left\langle(\lambda 3) \kappa_{1} L_{1} ;(10) 1 \|(\lambda-1,4) K L\right\rangle$ involving orthogonal states on the left but non-orthogonalized states $|(\lambda-1,4) K L M\rangle$ on the right (see table 5A). From the given expression for these coefficients, it is easy to make the transformation to the SU(3) Wigner coefficients for specific $\lambda$ and $L$ via eqs. (10a) and (10b).

To obtain coefficients of type (ii) it is more convenient to calculate $\langle(\lambda 3) \kappa L$; $(01) 1\left|(\lambda 4) \kappa_{1} L_{1}\right\rangle$ from tables of scalar products $\left\langle(\lambda 3) \kappa L ;(01) 1 \|(\lambda 4) K_{1} L_{1}\right\rangle$ again involving non-orthogonalized states $K_{1}$ on the right (table 5D). The coefficients $\left\langle(\lambda 4) \kappa_{1} L_{1} ;(10) 1 \|(\lambda 3) \kappa L\right\rangle$ can be obtained from above via the symmetry property (25a) to be given in sect. 8. Coefficients of the type (iii) can be obtained in a similar way from $\left\langle(\lambda+1,4) \kappa L ;(01) 1 \|(\lambda 4) \kappa_{1} L_{1}\right\rangle$, while the latter is obtained from the known coefficients (i) and (ii) through application of the building-up process (eq. 13), namely

$$
\begin{aligned}
& \left\langle(\lambda+1,4) \kappa L ;(01) 1 \|(\lambda 4) \kappa_{1} L_{1}\right\rangle U((\lambda+1,4)(10)(\lambda 4)(10) ;(\lambda+1,3)(01)) \\
& =\sum_{\kappa^{\prime}, L^{\prime}}\left\langle(\lambda+1,4) \kappa L ;(10) 1 \|(\lambda+1,3) \kappa^{\prime} L^{\prime}\right\rangle\left\langle(\lambda+1,3) \kappa^{\prime} L^{\prime} ;(10) 1 \|(\lambda 4) \kappa_{1} L_{1}\right\rangle \\
& \quad \times\langle(10) 1 ;(10) 1 \|(01) 1\rangle U\left(L 1 L_{1} 1 ; L^{\prime} 1\right) .
\end{aligned}
$$

In all cases (i) and (ii), we have given the SU(3) $\supset \mathrm{R}(3)$ coefficients for $L \leqq 3, \lambda$ arbitrary, which are of greatest importance in actual calculations. They are given in tables $5 \mathrm{~B}$ and $\mathrm{E}$.

Coefficients with $\lambda \leqq 4$, arbitrary $\mu$, follow from the above by employing the $\bar{\kappa}$ basis and using symmetry property (25c) given in sect. 8 . Coefficients with both $\lambda \geqq 5$ 
and $\mu \geqq 5$ can be calculated numerically employing techniques similar to the ones used to calculate the algebraic expressions for smaller $\mu$.

\section{Coefficients $(\lambda \mu) \times(20) \rightarrow\left(\lambda^{\prime} \mu^{\prime}\right)$ and $(\lambda \mu) \times(11) \rightarrow\left(\lambda^{\prime} \mu^{\prime}\right)$}

The coefficients for the products $(\lambda \mu) \times(20)$ and $(\lambda \mu) \times(11)$ are most easily calculated via the building-up process. The former are of importance for $2 \mathrm{~s}-1 \mathrm{~d}$ shell calculations. Eq. (14) with $\left(\lambda_{2} \mu_{2}\right)=(20)$ becomes

$$
\begin{aligned}
& \left\langle\left(\dot{\lambda}_{1} \mu_{1}\right) \kappa_{1} L_{1} ;(20) l||(\lambda \mu) \kappa L\right\rangle U\left(\left(\lambda_{1} \mu_{1}\right)(10)(\lambda \mu)(10) ;\left(\lambda^{\prime} \mu^{\prime}\right)(20)\right)=\sum_{\kappa^{\prime}, L^{\prime}} U\left(L_{1} 1 L 1 ; L^{\prime} l\right) \\
& \quad \times\left\langle\left(\lambda_{1} \mu_{1}\right) \kappa_{1} L_{1} ;(10) 1 \|\left(\lambda^{\prime} \mu^{\prime}\right) \kappa^{\prime} L^{\prime}\right\rangle\left\langle\left(\lambda^{\prime} \mu^{\prime}\right) \kappa^{\prime} L^{\prime} ;(10) 1 \|(\lambda \mu) \kappa L\right\rangle\langle(10) 1 ;(10) 1 \|(20) l\rangle .
\end{aligned}
$$

To facilitate the calculations, the simplest possible $\left(\lambda^{\prime} \mu^{\prime}\right)$ can be chosen. Since all intermediate products are simply reducible, no $\rho$-index is necessary. Coefficients of this nature were calculated for $\mu_{1} \leqq 3, \mu \leqq 3$ (arbitrary $\lambda_{1}, \lambda$ ). Coefficients with $\lambda_{1} \leqq 3, \lambda \leqq 3$ (arbitrarily large $\mu, \mu_{1}$ ) can be obtained from these by employing the $\kappa$-basis and using the symmetry property $(25 \mathrm{c})$ given in sect. 8 . Similarly

$\sum_{\rho}\left\langle\left(\lambda_{1} \mu_{1}\right) \kappa_{1} L_{1} ;(11) l \|(\hat{\lambda} \mu) \kappa L\right\rangle_{\rho} U\left(\left(\lambda_{1} \mu_{1}\right)(01)(\hat{\lambda} \mu)(10) ;\left(\lambda^{\prime} \mu^{\prime}\right)(11)\right)_{\rho}=\sum_{\kappa^{\prime} L^{\prime}} U\left(L_{1} 1 L 1 ; L^{\prime} l\right)$

$\times\left\langle\left(\lambda_{1} \mu_{1}\right) \kappa_{1} L_{1} ;(01) 1 \|\left(\lambda^{\prime} \mu^{\prime}\right) \kappa^{\prime} L^{\prime}\right\rangle\left\langle(\lambda \mu) \kappa^{\prime} L^{\prime} ;(10) 1 \|(\lambda \mu) \kappa L\right\rangle\langle(01) 1 ;(10) 1 \|(11) l\rangle,(20 \mathrm{a})$

or

$\sum_{\rho}\left\langle\left(\hat{\lambda}_{1} \mu_{1}\right) \kappa_{1} L_{1} ;(11) l \|(\lambda \mu) \kappa L\right\rangle_{\rho} U\left(\left(\lambda_{1} \mu_{1}\right)(10)(\lambda \mu)(01) ;\left(\hat{\lambda}^{\prime} \mu^{\prime}\right)(11)\right)_{\rho}=\sum_{\kappa^{\prime}, L^{\prime}} U\left(L_{1} 1 L 1 ; L^{\prime} l\right)$

× $\left\langle\left(\lambda_{1} \mu_{1}\right) \kappa_{1} L_{1} ;(10) 1||\left(\lambda^{\prime} \mu^{\prime}\right) \kappa^{\prime} L^{\prime}\right\rangle\left\langle\left(\lambda^{\prime} \mu^{\prime}\right) \kappa^{\prime} L^{\prime} ;(01) 1 \|(\lambda \mu) \kappa L\right\rangle\langle(10) 1 ;(01) 1 \|(11) l\rangle$,

whichever happens to allow a simpler choice of $\left(\lambda^{\prime} \mu^{\prime}\right)$. The index $\rho$ is necessary only when $(\lambda \mu)=\left(\lambda_{1} \mu_{1}\right), \mu_{1} \neq 0$. The number of required calculations is reduced because of the symmetry property (25b), and the fact that the representation (11) is selfcontragredient.

As a further illustration the build-up method will also be used for the calculation of a coefficient needed for two particle c.f.p. in the $2 \mathrm{~s}-1 \mathrm{~d}$ shell, namely

$$
\begin{aligned}
& \left\langle(\lambda 0) L_{1} ;(40) l \|(\lambda+4,0) L\right\rangle=\sum_{L^{\prime}, l_{1}, l_{2}}\left\langle(\lambda 0) L_{1} ;(20) l_{1} \|(\lambda+2,0) L^{\prime}\right\rangle \\
& \quad \times\left\langle(\lambda+2,0) L^{\prime} ;(20) l_{2} \|(\lambda+4,0) L\right\rangle\left\langle(20) l_{1} ;(20) l_{2} \|(40) l\right\rangle U\left(L_{1} l_{1} L l_{2} ; L^{\prime} l\right) .
\end{aligned}
$$

In this special case, the SU(3) $U$-function for the left-hand side has the value of +1 . Other coefficients of this type can be obtained in a similar way. 


\section{7. $\mathrm{SU}(3) \supset \mathbf{R}(3)$ tensor operators}

Irreducible tensor operators in the above scheme are defined in the usual way ${ }^{20,21}$ )

$$
\begin{array}{cc}
{\left[L_{v}, T_{\kappa L M}^{(\lambda \mu)}\right]=\sum_{\kappa^{\prime}, L^{\prime}}\left\langle(\lambda \mu) \kappa^{\prime} L^{\prime} M+v\left|L_{v}\right|(\lambda \mu) \kappa L M\right\rangle T_{\kappa^{\prime} L^{\prime} M+v}^{(\lambda \mu)},} & v=+1,0,-1, \\
{\left[Q_{\rho}, T_{\kappa L M}^{(\lambda \mu)}\right]=\sum_{\kappa^{\prime}, L^{\prime}}\left\langle(\lambda \mu) \kappa^{\prime} L^{\prime} M+\rho\left|Q_{\rho}\right|(\lambda \mu) \kappa L M\right\rangle T_{\kappa^{\prime} L^{\prime} M+\rho}^{(\lambda \mu)},} & \rho=0, \pm 1, \pm 2,
\end{array}
$$

$L_{v}$ and $Q_{\rho}$ were defined in sect. 2 .

From the above definition, it is clear that $L_{v}$ and $Q_{\rho}$ are themselves $\mathrm{SU}(3) \supset \mathrm{R}(3)$ tensors and transform according to the regular representation (11) of SU(3). Since phases and normalization are of some importance in some cases, the tensor character of $L_{v}, Q_{\rho}$ is given explicitly

$$
\begin{aligned}
T_{11}^{(11)} & =-\frac{1}{\sqrt{ } 2} L_{+}, \quad T_{10}^{(11)}=L_{0}, \quad T_{1-1}^{(11)}=\frac{1}{\sqrt{ } 2} L_{-}, \\
T_{2 \rho}^{(11)} & =-\frac{1}{\sqrt{ } 3} Q_{\rho},
\end{aligned}
$$

$\kappa$ is redundant here.

The phases were chosen in such a way that $T_{1 v}^{(11)}$ coincide with the conventional angular momentum tensor operators. Via the Wigner-Eckart ${ }^{21}$ ) theorem, eq. (23) can be put into the form

$$
\begin{aligned}
{\left[T_{l m}^{(11)} T_{\kappa L M}^{(\lambda \mu)}\right]=} & \sum_{\kappa^{\prime}, L^{\prime}}\left\langle(\lambda \mu) \kappa L ;(11) l \|(\lambda \mu) \kappa^{\prime} L^{\prime}\right\rangle_{\rho=1}\left\langle(\lambda \mu)\left\|T^{(11)}\right\|(\lambda \mu)\right\rangle_{\rho=1} \\
& \times\langle L M l m \mid L m+M\rangle T_{\kappa^{\prime} L^{\prime} m+M}^{(\lambda \mu)} .
\end{aligned}
$$

The $\left\langle(\lambda \mu)|| T^{(11)} \mid(\lambda \mu)\right\rangle_{\rho=1}$ except possibly for a $(\lambda \mu)$ dependent phase factor is the same as the corresponding double-bar matrix element in the intrinsic scheme.

\section{Symmetry properties}

The SU(3) Wigner coefficients based on the $|(\lambda \mu) \kappa L M\rangle$ scheme have properties which are the analogues of the corresponding symmetry properties in the intrinsic scheme ${ }^{3,13}$ ). It is shown in the appendix that the following symmetry properties hold

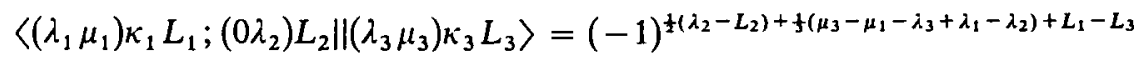

$$
\begin{aligned}
& \times\left\langle\left(\lambda_{3} \mu_{3}\right) \kappa_{3} L_{3} ;\left(\lambda_{2} 0\right) L_{2} \|\left(\lambda_{1} \mu_{1}\right) \kappa_{1} L_{1}\right\rangle\left[\frac{\operatorname{dim}\left(\lambda_{3} \mu_{3}\right)\left(2 L_{1}+1\right)}{\operatorname{dim}\left(\lambda_{1} \mu_{1}\right)\left(2 L_{3}+3\right)}\right]^{\frac{1}{2}},
\end{aligned}
$$

where $\operatorname{dim}(\lambda \mu)=\frac{1}{2}(\lambda+1)(\mu+1)(\lambda+\mu+2)$.

$$
\begin{aligned}
\left\langle\left(\lambda_{1} \mu_{1}\right) \kappa_{1} L_{1}\right. & \left.;\left(1 \lambda_{2}\right) L_{2} \|\left(\lambda_{3} \mu_{3}\right) \kappa_{3} L_{3}\right\rangle=(-1)^{\delta}(-1)^{\frac{f}{\left(\mu_{3}+1-\mu_{1}-\lambda_{3}-\lambda_{2}+\lambda_{1}\right)+L_{1}-L_{3}}} \\
& \times\left\langle\left(\lambda_{3} \mu_{3}\right) \kappa_{3} L_{3} ;\left(\lambda_{2} 1\right) L_{2} \|\left(\lambda_{1} \mu_{1}\right) \kappa_{1} L_{1}\right\rangle\left[\frac{\operatorname{dim}\left(\lambda_{3} \mu_{3}\right)\left(2 L_{1}+1\right)}{\operatorname{dim}\left(\lambda_{1} \mu_{1}\right)\left(2 L_{3}+1\right)}\right]^{\frac{1}{3}}
\end{aligned}
$$


with $\delta=\frac{1}{2}\left[\lambda_{2}-L_{2}+1\right],[x]=$ integral part of $x$,

$$
\begin{aligned}
& \left\langle\left(\mu_{1} \lambda_{1}\right) \bar{\kappa}_{1} L_{1} ;\left(\mu_{2} \lambda_{2}\right) \bar{\kappa}_{2} L_{2} \|\left(\mu_{3} \lambda_{3}\right) \bar{\kappa}_{3} L_{3}\right\rangle \\
& =\left\langle\left(\lambda_{1} \mu_{1}\right) \kappa_{1} L_{1} ;\left(\lambda_{2} \mu_{2}\right) \kappa_{2} L_{2} \|\left(\lambda_{3} \mu_{3}\right) \kappa_{3} L_{3}\right\rangle(-1)^{\lambda_{1}+\lambda_{2}-\lambda_{3}} .
\end{aligned}
$$

It is a pleasure indeed to acknowledge here my most sincere thanks to Professor K. T. Hecht, Chairman of my Doctoral Committee, for suggesting the problem, for his guidance, thoughtful encouragement and generous use of his time in enlightening discussions. I would also like to thank T. Engeland for stimulating discussions, P. J. Ellis for helpful suggestions and W. C. Parkinson for supporting this work.

Note added while manuscript was under preparation:

Recently Iosifescu and Stancu ${ }^{24}$ ) have calculated $\mathrm{SU}(3) \supset \mathbf{R}(3)$ Wigner coefficients for the special cases

$$
\begin{aligned}
& \left\langle(\lambda-2,0) l^{\prime} ;(20) l^{\prime \prime} \|(\lambda 0) l\right\rangle, \quad\left\langle(\lambda-2,0) l^{\prime} ;(20) l^{\prime \prime} \|(\lambda-2,1) l\right\rangle, \\
& \left\langle(\lambda-4,1) l^{\prime} ;(20) l^{\prime \prime} \|(\lambda-2,1) l\right\rangle .
\end{aligned}
$$

They are part of tables 6 and 7 of the present work and have been included since their phase convention is different from the present one.

\section{Appendix 1}

\section{A.1. SYMMETRY PROPERTIES (25a) AND (25b)}

The symmetry properties which are of interest in the present paper can be obtained if transformation properties of $|(\lambda \mu) \kappa L M\rangle$ under complex conjugation are known. One such conjugation relation is given by eq. $(10 \mathrm{c})$. However this relates the states $\kappa$ of $(\lambda \mu)$ to the states $\bar{\kappa}$ of the conjugate representation $(\mu \lambda)$. Since the states $\bar{\kappa}$ are in general complicated functions of the states $\kappa$, conjugation properties relating the states $\kappa$ of $(\lambda \mu)$ to the states $\kappa$ of $(\mu \lambda)$ are very complicated in the general case. However in cases where there is no $L$-multiplicity we must have

$$
|(\lambda \mu) \kappa L M\rangle^{*}=i^{\beta}|(\mu \lambda) \kappa L-M\rangle .
$$

Since the transformation properties of the $R(3)$ part are well known, one can factor out the $R(3)$ dependence of the phase and write

$$
|(\lambda \mu) \kappa L M\rangle^{*}=i^{\alpha}(-1)^{L-M}|(\mu \lambda) \kappa L-M\rangle
$$

where $\alpha$ is now independent of $M ; \alpha=\alpha(\lambda, \mu, L)$. The phase $\alpha$ in eq. (A.1) was found to be $\alpha=2 \cdot \frac{1}{2}(\lambda+L)$ for $\mu=0$ and $\alpha=2 \cdot \frac{1}{2}[\lambda+L+1]$ for $\mu=1$, where $[X]$ is the integral part of $X$, i.e. $\alpha$ is an even integer. In what follows for compactness of notation, we shall write $\left(\lambda_{2} \mu_{2}\right)$, but what we have in mind is $\left(\lambda_{2} \mu_{2}\right)=\left(\lambda_{2} 0\right)$ or $(11)$. 
Let us define an operator $a_{\left(\lambda_{2} \mu_{2}\right) \kappa_{2} L_{2} M_{2}}^{+}$such that

$$
\left|\left(\lambda_{2} \mu_{2}\right) \kappa_{2} L_{2} M_{2}\right\rangle=a_{\left(\lambda_{2} \mu_{2}\right) \kappa_{2} L_{2} M_{2}}^{+}|0\rangle,
$$

where $|0\rangle$ is the closed shell "vacuum", i.e. $3 N$ oscillator quanta coupled to $(\lambda \mu)=(00)$. E.g. the single-particle creation operator $a_{\left(\lambda_{2} O\right) L_{2} M_{2}}^{+}$is an operator which creates a particle in the $\lambda_{2}$ th major oscillator shell with angular momentum $L_{2} M_{2}$. The meaning of definition (A.2) can now be made clear since any operator of the form introduced there can be expressed in terms of these single-particle operators. Since the state $\left|\left(\lambda_{2} \mu_{2}\right) \kappa_{2} L_{2} M_{2}\right\rangle$ has the same transformation properties under $\mathrm{SU}(3)$ as a tensor $T_{\kappa_{2} L_{2} M_{2}}^{\left(\lambda_{2} \mu_{2}\right)}$ and since $|0\rangle$ transforms like a scalar under $\mathrm{SU}(3)$, i.e. it remains unchanged, it follows that $a_{\left(\lambda_{2} \mu_{2}\right) \kappa_{2} L_{2} M_{2}}^{+}$transforms like $T_{\kappa_{2} L_{2} M_{2}}^{\left(\lambda_{2} \mu_{2}\right)}$. Therefore we can write

Then from eq. (A.1)

$$
a_{\left(\lambda_{2} \mu_{2}\right) \kappa_{2} L_{2} M_{2}}^{+} \equiv T_{\kappa_{2} L_{2} M_{2}}^{\left(\lambda_{2} \mu_{2}\right)} \text {. }
$$

$$
\left(a_{\left(\lambda_{2} \mu_{2}\right) \kappa_{2} L_{2} M_{2}}^{+}\right)^{+}=i^{\alpha}(-1)^{L_{2}-M_{2}} T_{\kappa_{2} L_{2}-M_{2}}^{\left(\mu_{2} \lambda_{2}\right)} .
$$

The tensor $T_{\kappa_{2} L_{2}-M_{2}}^{\left(\mu_{2} \lambda_{2}\right)}$ transforms contragrediently to the tensor $T_{\kappa_{2} L_{2} M_{2}}^{\left(\lambda_{2} \mu_{2}\right)}$ under SU(3).

It then follows that

$$
\begin{aligned}
\left\langle\left(\lambda_{3} \mu_{3}\right) \kappa_{3} L_{3} M_{3}\left|a_{\left(\lambda_{2} \mu_{2}\right) \kappa_{2} L_{2} M_{2}}^{+}\right|\left(\lambda_{1} \mu_{1}\right) \kappa_{1} L_{1} M_{1}\right\rangle=\left\langle\left(\lambda_{3} \mu_{3}\right)|| T^{\left(\lambda_{2} \mu_{2}\right)} \|\left(\lambda_{1} \mu_{1}\right)\right\rangle \\
\quad \times\left\langle\left(\lambda_{1} \mu_{1}\right) \kappa_{1} L_{1} M_{1} ;\left(\lambda_{2} \mu_{2}\right) \kappa_{2} L_{2} M_{2} \mid\left(\lambda_{3} \mu_{3}\right) \kappa_{3} L_{3} M_{3}\right\rangle \\
\left\langle\left(\lambda_{1} \mu_{1}\right) \kappa_{1} L_{1}\left|T_{\kappa_{2} L_{2}-M_{2}}^{\left(\mu_{2} \lambda_{2}\right)}\right|\left(\lambda_{3} \mu_{3}\right) \kappa_{3} L_{3} M_{3}\right\rangle \\
=\left\langle\left(\lambda_{3} \mu_{3}\right) \kappa_{3} L_{3} M_{3} ;\left(\mu_{2} \lambda_{2}\right) \kappa_{2} L_{2}-M_{2} \mid\left(\lambda_{1} \mu_{1}\right) \kappa_{1} L_{1} M_{1}\right\rangle \\
\quad \times\left\langle\left(\lambda_{1} \mu_{1}\right) \| T^{\left(\mu_{2} \lambda_{2}\right)}||\left(\lambda_{3} \mu_{3}\right)\right\rangle .
\end{aligned}
$$

The index $\rho$ was omitted here because, for the cases $\left(\lambda_{2} \mu_{2}\right)$ considered, only one reduced matrix element is non-zero in the case $\left(\lambda_{2} \mu_{2}\right)=(11)$ by choice of $\rho$ [ref. $\left.{ }^{3}\right)$ ]. From the reality of the $S U(3) \supset R(3)$ Wigner coefficients and (A.4), one gets

$$
\begin{aligned}
& \left\langle\left(\lambda_{1} \mu_{1}\right) \kappa_{1} L_{1} M_{1}\left|T_{\kappa_{2} L_{2}-M_{2}}^{\left(\mu_{2} \lambda_{2}\right)}\right|\left(\lambda_{3} \mu_{3}\right) \kappa_{3} L_{3} M_{3}\right\rangle \\
& \quad=(-i)^{\alpha}(-1)^{L_{2}-M_{2}}\left\langle\left(\lambda_{3} \mu_{3}\right) \kappa_{3} L_{3} M_{3}\left|T_{\kappa_{2} L_{2} M_{2}}^{\left(\lambda_{2} \mu_{2}\right)}\right|\left(\lambda_{1} \mu_{1}\right) \kappa_{1} L_{1} M_{1}\right\rangle,
\end{aligned}
$$

or

$$
\begin{gathered}
\frac{\left\langle\left(\lambda_{1} \mu_{1}\right) \kappa_{1} L_{1} M_{1} ;\left(\lambda_{2} \mu_{2}\right) \kappa_{2} L_{2} M_{2} \mid\left(\lambda_{3} \mu_{3}\right) \kappa_{3} L_{3} M_{3}\right\rangle}{\left\langle\left(\lambda_{3} \mu_{3}\right) \kappa_{3} L_{3} M_{3} ;\left(\mu_{2} \lambda_{2}\right) \kappa_{2} L_{2}-M_{2} \mid\left(\lambda_{1} \mu_{1}\right) \kappa_{1} L_{1} M_{1}\right\rangle} \\
=(-i)^{\alpha}(-1)^{L_{2}-M_{2}} \frac{\left\langle\left(\lambda_{1} \mu_{1}\right) \| T^{\left(\mu_{2} \lambda_{2}\right)}||\left(\lambda_{3} \mu_{3}\right)\right\rangle}{\left\langle\left(\lambda_{3} \mu_{3}\right) \| T^{\left(\lambda_{2} \mu_{2}\right)}||\left(\lambda_{1} \mu_{1}\right)\right\rangle} .
\end{gathered}
$$

But from the symmetry property in the intrinsic scheme analogous to properties (25a) and (25b) and a relation in the intrinsic scheme analogous to (A.6) found in appendix $\mathrm{A} 3$ of ref. $^{3}$ ), one gets

$$
\frac{\left\langle\left(\lambda_{1} \mu_{1}\right)|| T^{\left(\mu_{2} \lambda_{2}\right)} \|\left(\lambda_{3} \mu_{3}\right)\right\rangle}{\left\langle\left(\lambda_{3} \mu_{3}\right)\left\|T^{\left(\lambda_{2} \mu_{2}\right)}\right\|\left(\lambda_{1} \mu_{1}\right)\right\rangle}=(-1)^{\frac{1}{\left(\mu_{1}+\mu_{2}-\mu_{3}-\lambda_{1}-\lambda_{2}+\lambda_{3}\right)}}\left[\frac{\operatorname{dim}\left(\lambda_{3} \mu_{3}\right)}{\operatorname{dim}\left(\hat{\lambda}_{1} \mu_{1}\right)}\right]^{\frac{1}{2}} \text {. }
$$


Hence one finds

$$
\begin{aligned}
& \left\langle\left(\lambda_{3} \mu_{3}\right) \kappa_{3} L_{3} M_{3} ;\left(\mu_{2} \lambda_{2}\right) \kappa_{2} L_{2}-M_{2} \mid\left(\lambda_{1} \mu_{1}\right) \kappa_{1} L_{1} M_{1}\right\rangle \\
& =(-i)^{\alpha}(-1)^{f\left(\mu_{1}+\mu_{2}-\mu_{3}-\lambda_{1}-\lambda_{2}+\lambda_{3}\right)+L_{2}-M_{2}} \\
& \times\left[\frac{\operatorname{dim}\left(\lambda_{1} \mu_{1}\right)}{\operatorname{dim}\left(\lambda_{3} \mu_{3}\right)}\right]^{\frac{1}{2}}\left\langle\left(\lambda_{1} \mu_{1}\right) \kappa_{1} L_{1} M_{1} ;\left(\lambda_{2} \mu_{2}\right) \kappa_{2} L_{2} M_{2} \mid\left(\lambda_{3} \mu_{3}\right) \kappa_{3} L_{3} M_{3}\right\rangle .
\end{aligned}
$$

Factoring out the $\mathrm{R}(3)$ part and using the well-known symmetry property for the $\mathrm{R}(3)$ Wigner coefficients, we get

$$
\begin{aligned}
& \left\langle\left(\lambda_{3} \mu_{3}\right) \kappa_{3} L_{3} ;\left(\mu_{2} \lambda_{2}\right) \kappa_{2} L_{2} \|\left(\lambda_{1} \mu_{1}\right) \kappa_{1} L_{1}\right\rangle=(-i)^{\alpha}(-1)^{f\left(\mu_{1}+\mu_{2}-\mu_{3}-\lambda_{1}-\lambda_{2}+\lambda_{3}\right)+L_{1}-L_{3}+L_{2}} \\
& \times\left[\frac{\operatorname{dim}\left(\lambda_{1} \mu_{1}\right)\left(2 L_{3}+1\right)}{\operatorname{dim}\left(\lambda_{3} \mu_{3}\right)\left(2 L_{1}+1\right)}\right]^{\frac{1}{2}}\left\langle\left(\lambda_{1} \mu_{1}\right) \kappa_{1} L_{1} ;\left(\lambda_{2} \mu_{2}\right) \kappa_{2} L_{2} \|\left(\lambda_{3} \mu_{3}\right) \kappa_{3} L_{3}\right\rangle
\end{aligned}
$$

Since $\alpha$ is an even integer for the cases considered, we find relations (25a) and (25b) with $\delta=\frac{1}{2} \alpha-L_{2}$.

\section{A.2. SYMMETRY PROPERTY OF EQ. (24)}

From eq. (10c) and the reality of the SU(3) $\supset \mathrm{R}(3)$ Wigner coefficients, one expects a relation of the type

$$
\begin{aligned}
& \left\langle\left(\lambda_{1} \mu_{1}\right) \kappa_{1} L_{1} M_{1} ;\left(\lambda_{2} \mu_{2}\right) \kappa_{2} L_{2} M_{2} \mid\left(\lambda_{3} \mu_{3}\right) \kappa_{3} L_{3} M_{3}\right\rangle \\
& \quad=(-1)^{\gamma}\left\langle\left(\mu_{1} \lambda_{1}\right) \bar{\kappa}_{1} L_{1}-M_{1} ;\left(\mu_{2} \lambda_{2}\right) \kappa_{2} L_{2}-M_{2} \mid\left(\mu_{3} \lambda_{3}\right) \bar{\kappa}_{3} L_{3}-M_{3}\right\rangle
\end{aligned}
$$

where $\gamma$ is an integer.

To find $\gamma$, we conjugate the expression

$$
\begin{aligned}
\left|\left(\lambda_{3} \mu_{3}\right) \kappa_{3} L_{3} M_{3}\right\rangle_{\rho}= & \sum_{\substack{\kappa_{1} L_{1} M_{1} \\
\kappa_{2} L_{2}\left(M_{2}\right)}}\left\langle\left(\lambda_{1} \mu_{1}\right) \kappa_{1} L_{1} M_{1} ;\left(\lambda_{2} \mu_{2}\right) \kappa_{2} L_{2} M_{2} \mid\left(\lambda_{3} \mu_{3}\right) \kappa_{3} L_{3} M_{3}\right\rangle_{\rho} \\
& \times\left|\left(\lambda_{1} \mu_{1}\right) \kappa_{1} L_{1} M_{1}\right\rangle\left|\left(\lambda_{2} \mu_{2}\right) \kappa_{2} L_{2} M_{2}\right\rangle,
\end{aligned}
$$

and compare it via eq. (10c) with the relation

$$
\begin{aligned}
\left|\left(\mu_{3} \lambda_{3}\right) \bar{\kappa}_{3} L_{3}-M_{3}\right\rangle_{\rho}= & \sum_{\substack{\kappa_{1} L_{1} M_{1} \\
\kappa_{2} L_{2} M_{2}}}\left\langle\mu_{1} \lambda_{1}\right) \bar{\kappa}_{1} L_{1}-M_{1} ;\left(\mu_{2} \lambda_{2}\right) \bar{\kappa}_{2} L_{2}-M_{2}\left|\left(\mu_{3} \lambda_{3}\right) \bar{\kappa}_{3} L_{3}-\dot{M}_{3}\right\rangle_{\rho} \\
& \times\left|\left(\mu_{1} \lambda_{1}\right) \bar{\kappa}_{1} L_{1}-M_{1}\right\rangle\left|\left(\mu_{2} \lambda_{2}\right) \bar{\kappa}_{2} L_{2}-M_{2}\right\rangle .
\end{aligned}
$$

Then we find

$$
\gamma=\lambda_{1}+\lambda_{2}-\lambda_{3}+L_{1}+L_{2}-L_{3}
$$

factoring out the $R(3)$ dependence and using the corresponding symmetry for Clebsch-Gordan coefficients, we get

$$
\begin{aligned}
& \left\langle\left(\lambda_{1} \mu_{1}\right) \kappa_{1} L_{1} ;\left(\lambda_{2} \mu_{2}\right) \kappa_{2} L_{2}||\left(\lambda_{3} \mu_{3}\right) \kappa_{3} L_{3}\right\rangle \\
& =(-1)^{\lambda_{1}+\lambda_{2}-\lambda_{3}}\left\langle\left(\mu_{1} \lambda_{1}\right) \bar{\kappa}_{1} L_{1} ;\left(\mu_{2} \lambda_{2}\right) \bar{\kappa}_{2} L_{2} \mid\left(\mu_{3} \lambda_{3}\right) \bar{\kappa}_{3} L_{3}\right\rangle .
\end{aligned}
$$




\section{Appendix 2}

A USEFUL FORM FOR THE COEFFICIENTS $a((\lambda \mu) K L)$

First we calculate $A((\lambda 0) 0 L 0), \lambda-L=$ even. Eq. (6) becomes

$$
\begin{aligned}
A((\lambda 0) 0 L 0)= & \frac{2 L+1}{8 \pi^{2}} \int_{0}^{2 \pi} \mathrm{d} \alpha \int_{0}^{2 \pi} \mathrm{d} \gamma \int_{-1}^{1} \mathrm{~d} \xi \xi^{\lambda} P_{L}(\xi)=\frac{2 L+1}{2} \frac{\lambda(\lambda-1) \ldots(\lambda-L+1)}{2^{L} L !} \\
& \times \sum_{\alpha=0}^{L}(-1)^{\alpha}\left(\begin{array}{l}
L \\
\alpha
\end{array}\right) \frac{2}{\lambda-L+2 \alpha+1} .
\end{aligned}
$$

Theorem:

$$
\sum_{\alpha=0}^{L}(-1)^{\alpha}\left(\begin{array}{l}
L \\
\alpha
\end{array}\right) \frac{1}{\lambda-L+2 \alpha+1}=\frac{2^{L} L !}{(\lambda-L+1)(\lambda-L+3) \ldots(\lambda+L-1)(\lambda+L+1)} .
$$

Proof (by induction): The relation is obviously true for $L=1$.

Suppose it is true for $L=L$, then

$$
\begin{aligned}
\sum_{\alpha=0}^{L+1}(-1)^{\alpha}\left(\begin{array}{c}
L+1 \\
\alpha
\end{array}\right) \frac{1}{\lambda-(L+1)+2 \alpha+1}=\sum_{\alpha=1}^{L}(-1)^{\alpha}\left\{\left(\begin{array}{l}
L \\
\alpha
\end{array}\right)+\left(\begin{array}{c}
L \\
\alpha-1
\end{array}\right)\right\} \frac{1}{\lambda-1-L+2 \alpha+1} \\
+\frac{1}{\lambda-L}+(-1)^{L+1} \frac{1}{\lambda+L+2}=\sum_{\alpha=0}^{L}(-1)^{\alpha}\left(\begin{array}{l}
L \\
\alpha
\end{array}\right) \frac{1}{\lambda-1-L+2 \alpha+1} \\
\quad-\sum_{\alpha=0}^{L}(-1)^{\alpha}\left(\begin{array}{l}
L \\
\alpha
\end{array}\right) \frac{1}{(\lambda+1)-L+2 \alpha+1} \\
=\frac{2^{L} L !}{(\lambda-L+2)(\lambda-L+4) \ldots(\lambda+L)}\left\{\frac{1}{\lambda-L}-\frac{1}{\lambda+L+2}\right\} \\
=\frac{2^{L+1}(L+1) !}{(\lambda-L)(\lambda-L+2)(\lambda-L+4) \ldots(\lambda+L+2)} \text { q.e.d. }
\end{aligned}
$$

Use was made of the identity of combinatorial analysis

Hence

$$
\left(\begin{array}{l}
n \\
l
\end{array}\right)=\left(\begin{array}{c}
n-1 \\
l
\end{array}\right)+\left(\begin{array}{c}
n-1 \\
l-1
\end{array}\right)
$$

$$
\begin{aligned}
A((\lambda 0) 0 L 0) & =(2 L+1) b(\lambda, L), \\
b(\lambda, L) & =\frac{\lambda(\lambda-1) \ldots(\lambda-L+1)}{(\lambda-L+1)(\lambda-L+3) \ldots(\lambda+L-1)(\lambda+L+1)}, \quad L \geqq 1, \\
b(\lambda, 0) & =\frac{1}{\lambda+1} .
\end{aligned}
$$

In case $\mu \neq 0$ the corresponding integrals are more complicated. However, making use of the relation between the $d_{K^{\prime} K}^{L}(\xi)$ and the corresponding Jacobi polynomials found in the standard references ${ }^{22,23}$ ), it is possible to write the integral (6) as a sum of integrals of the form (A.10). However, what remains in the summation, after the common factors have been taken out, is an irreducible polynomial in $\lambda$ and $L$ whose degree increases with increasing $\mu$. The coefficients $(a(\lambda \mu) K L)$ for $\mu \leqq 4$ are given in table $2 \mathrm{~A}$. 


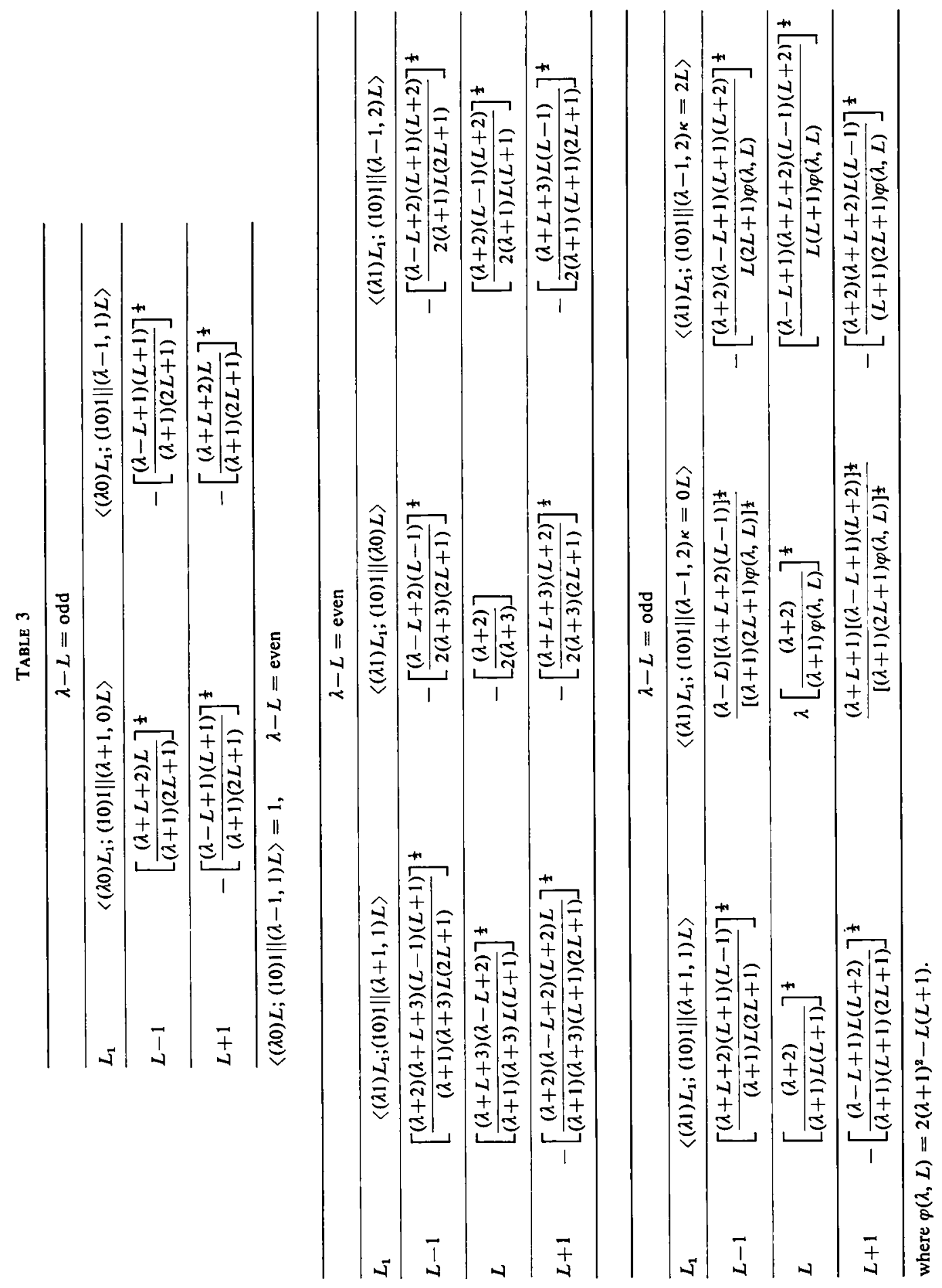




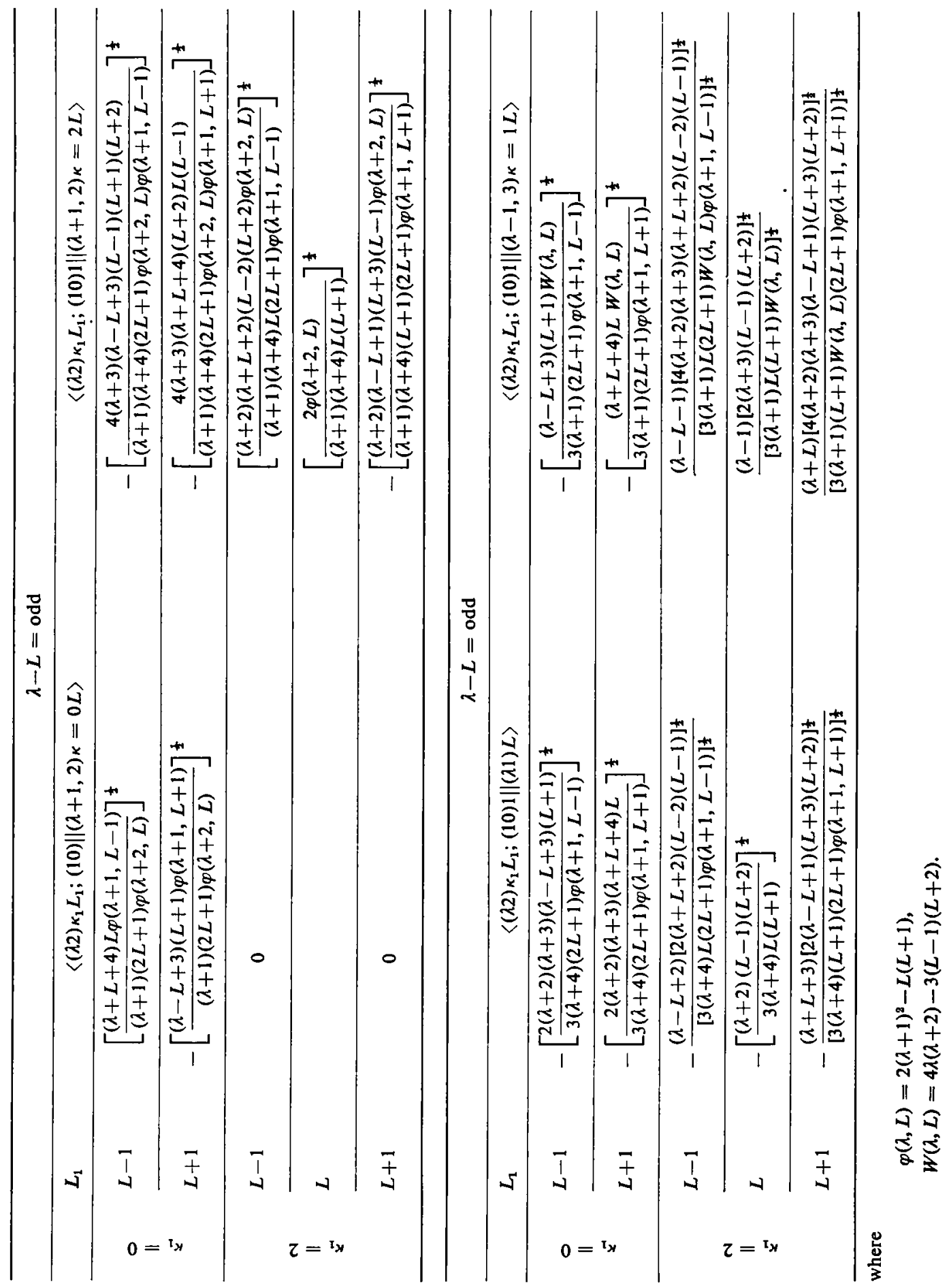




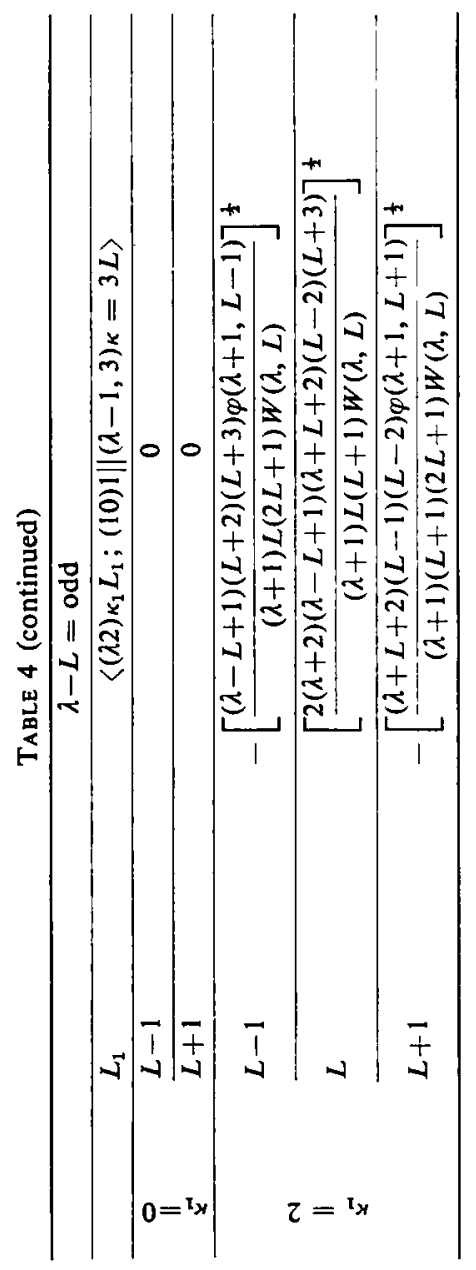

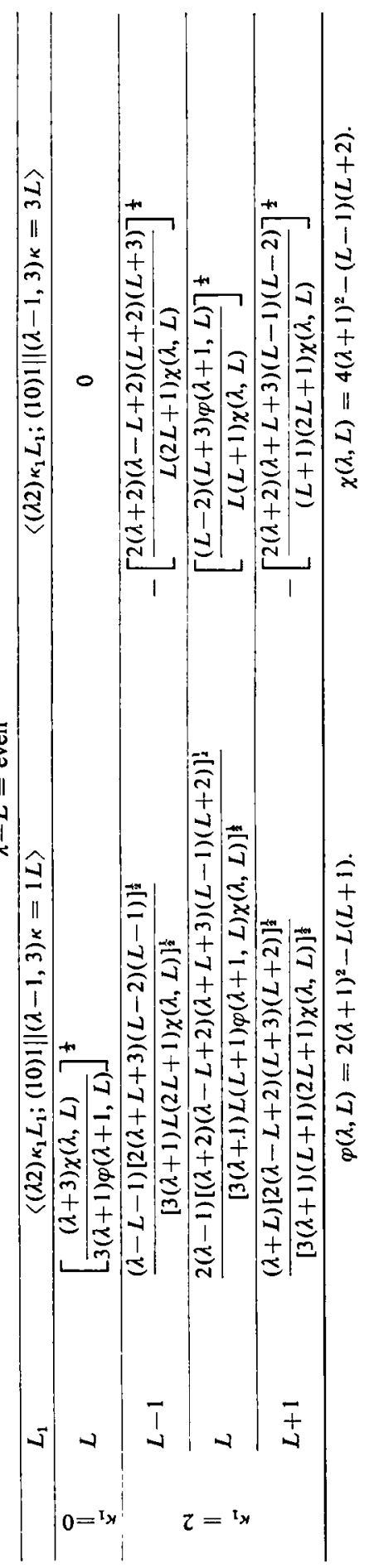




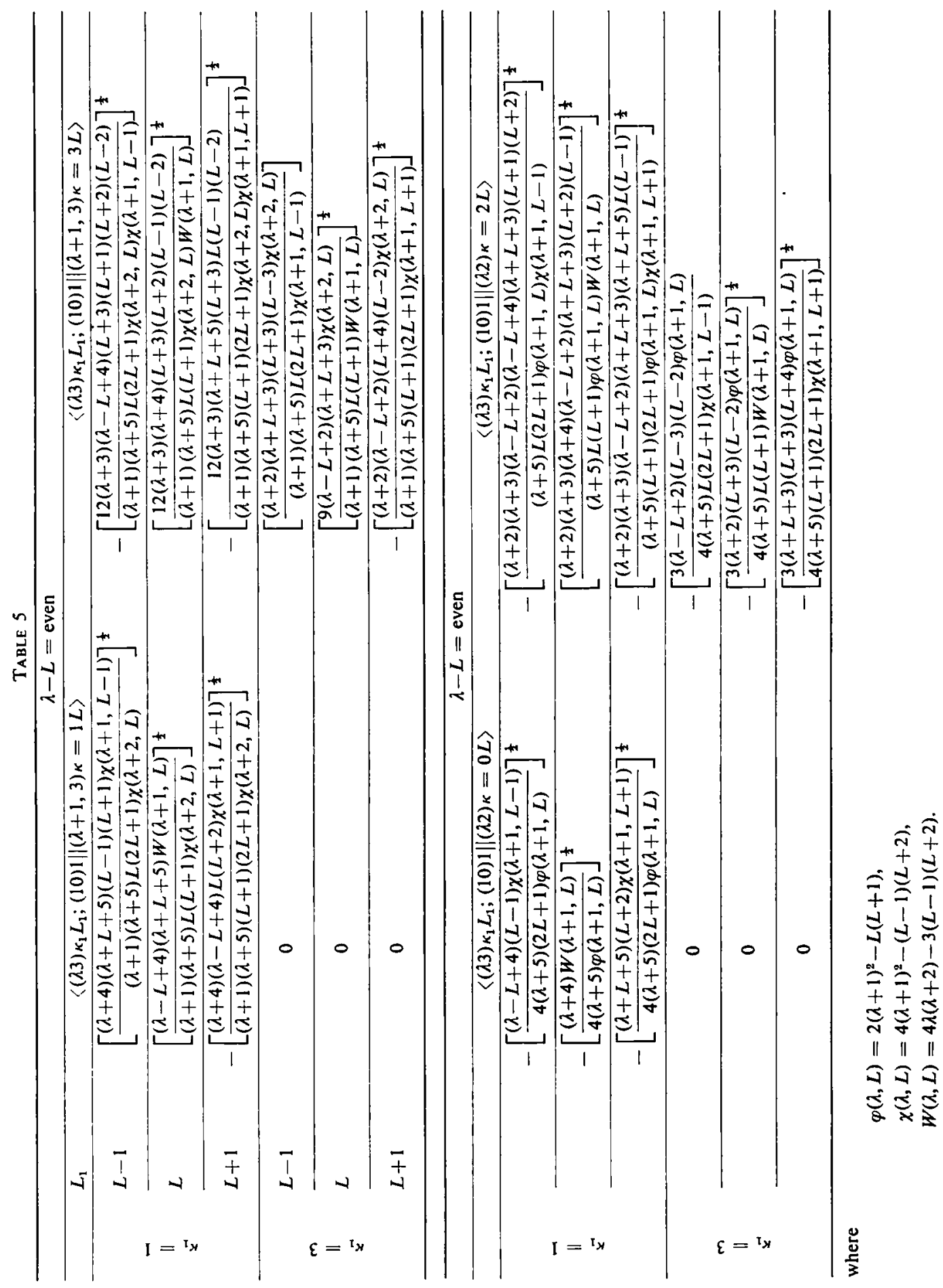




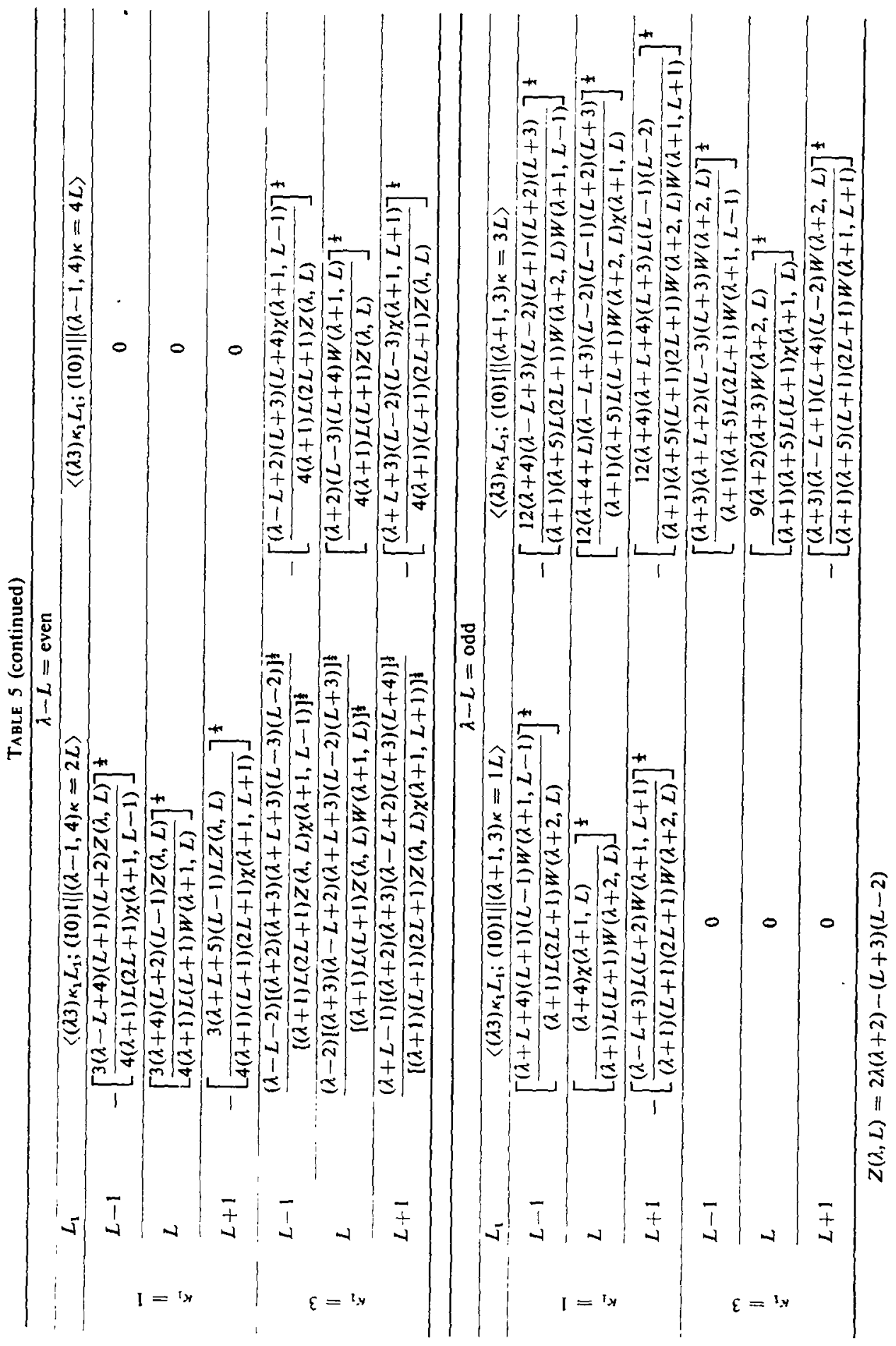




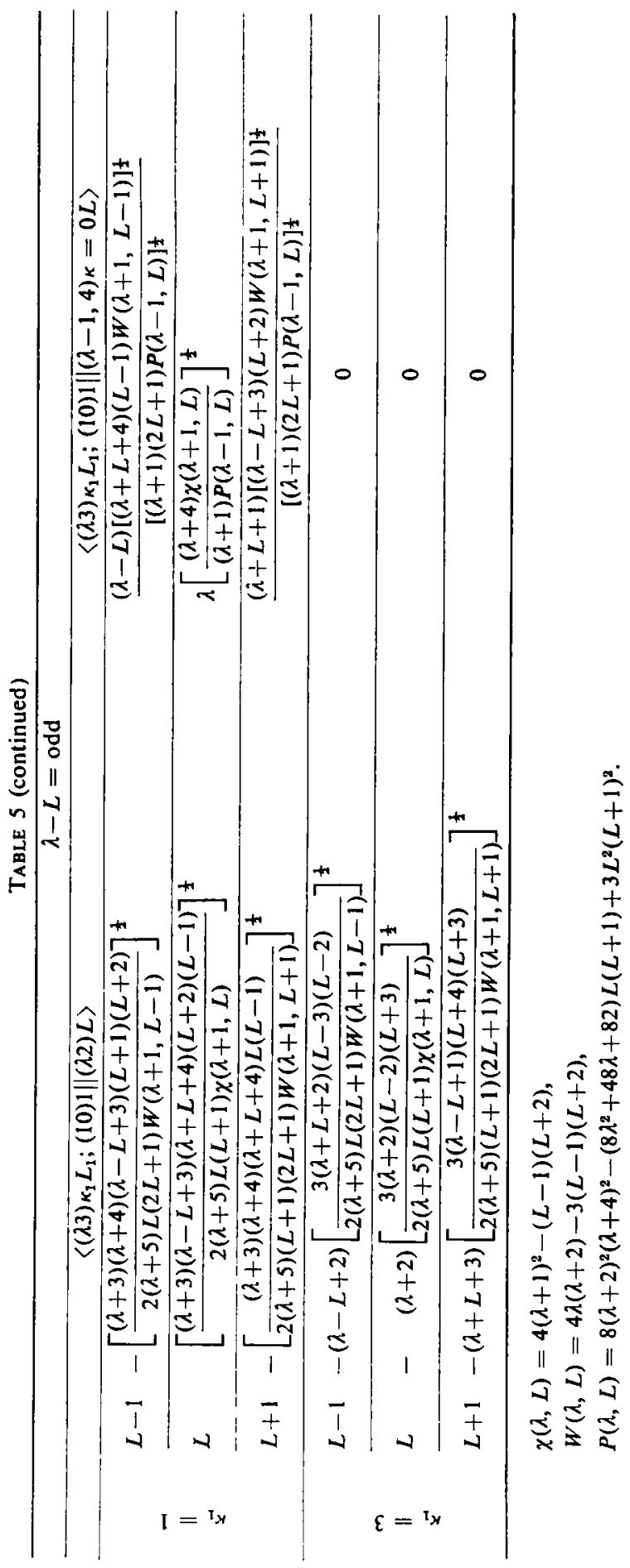




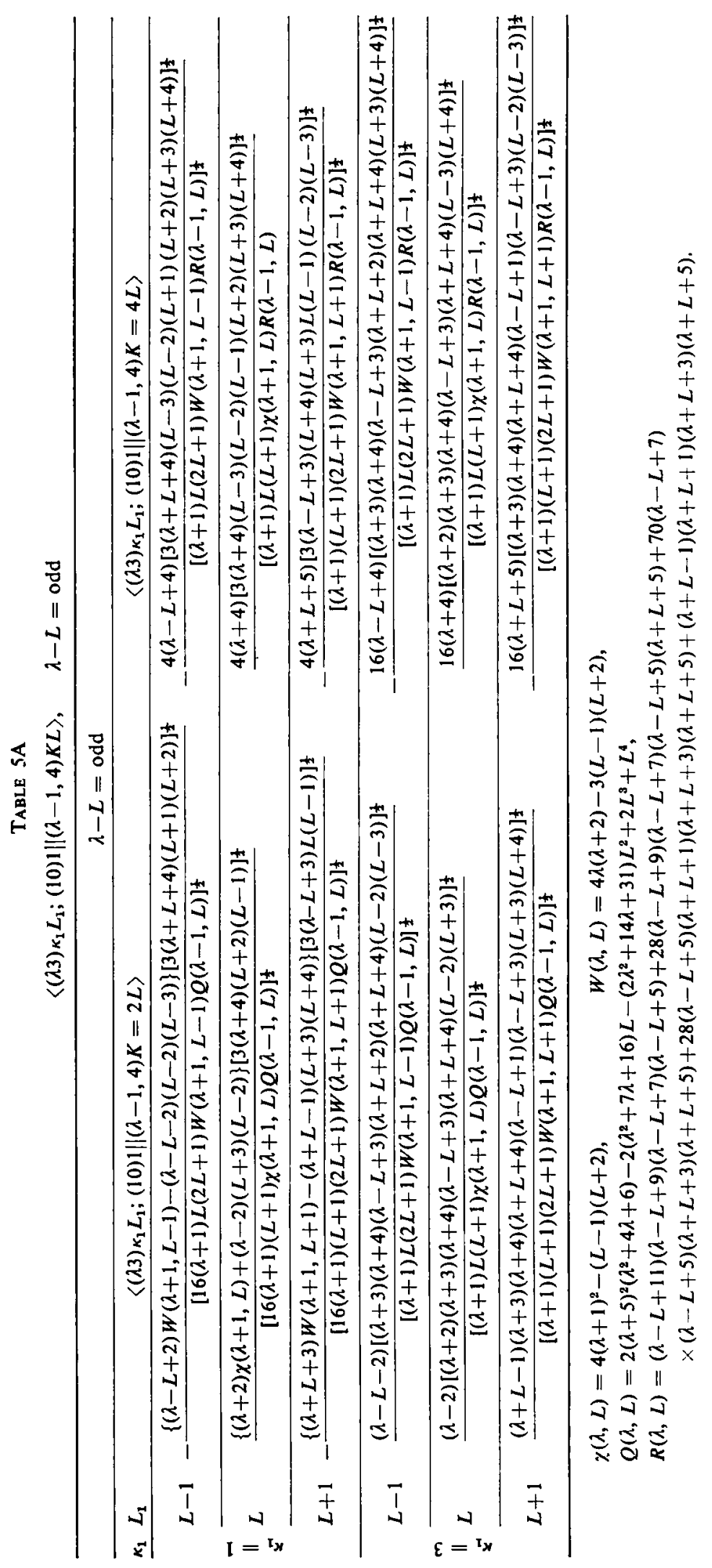




\section{TABle 5B}

$\left\langle(\lambda 3) \kappa_{1} L_{1} ;(10) 1 \|(\lambda-1,4) \kappa L\right\rangle, \quad \lambda-L=$ odd with $L \leqq 3$

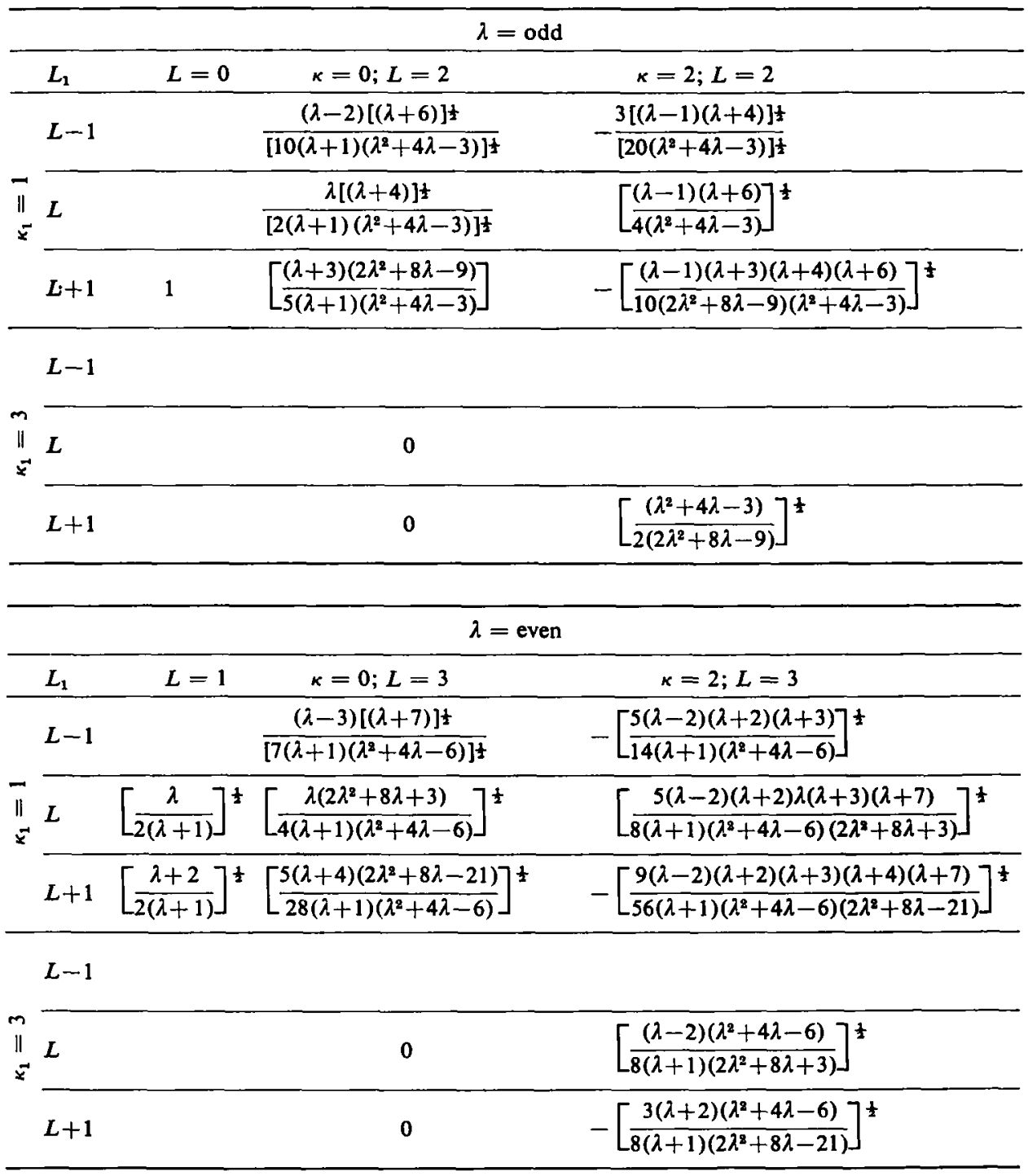




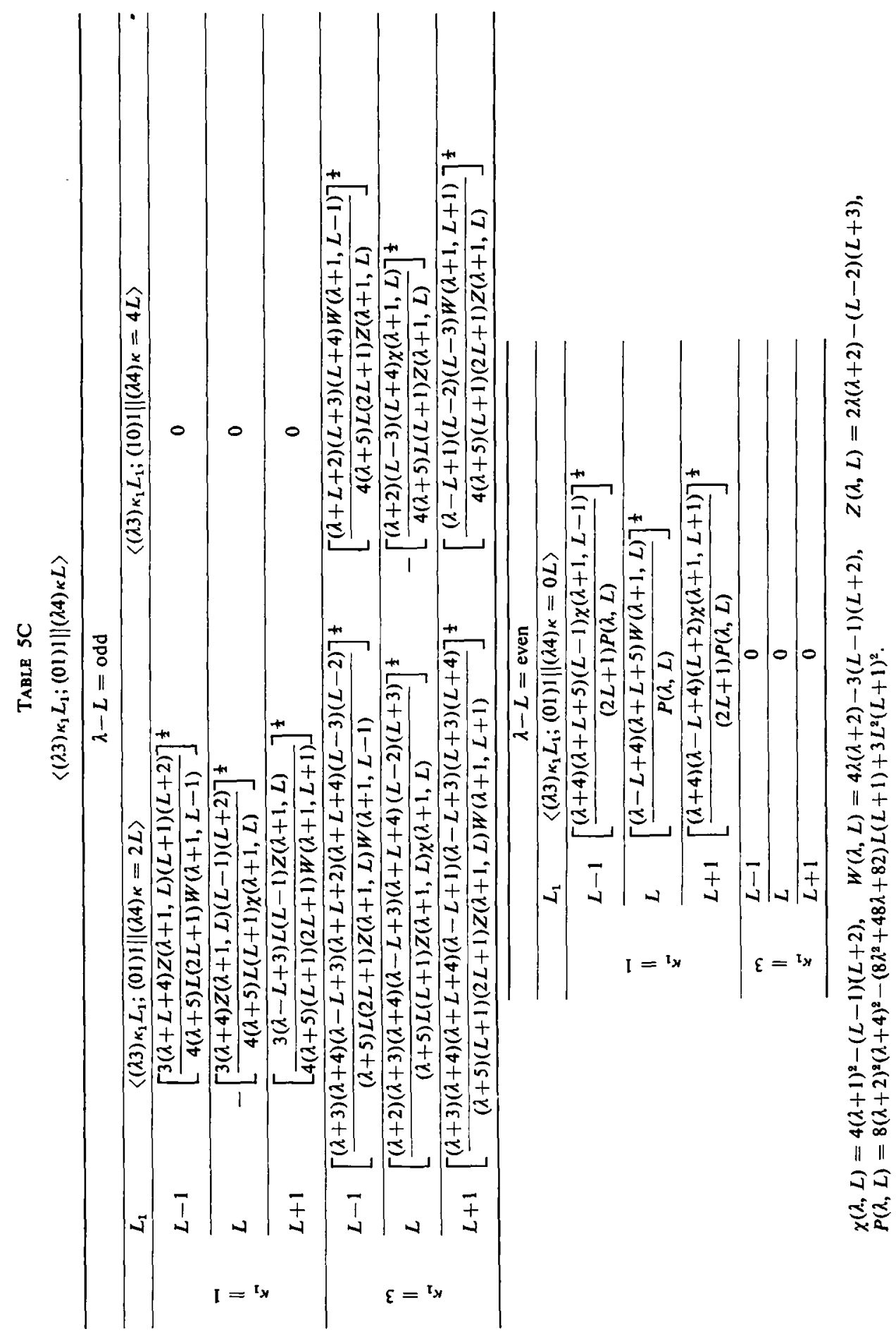




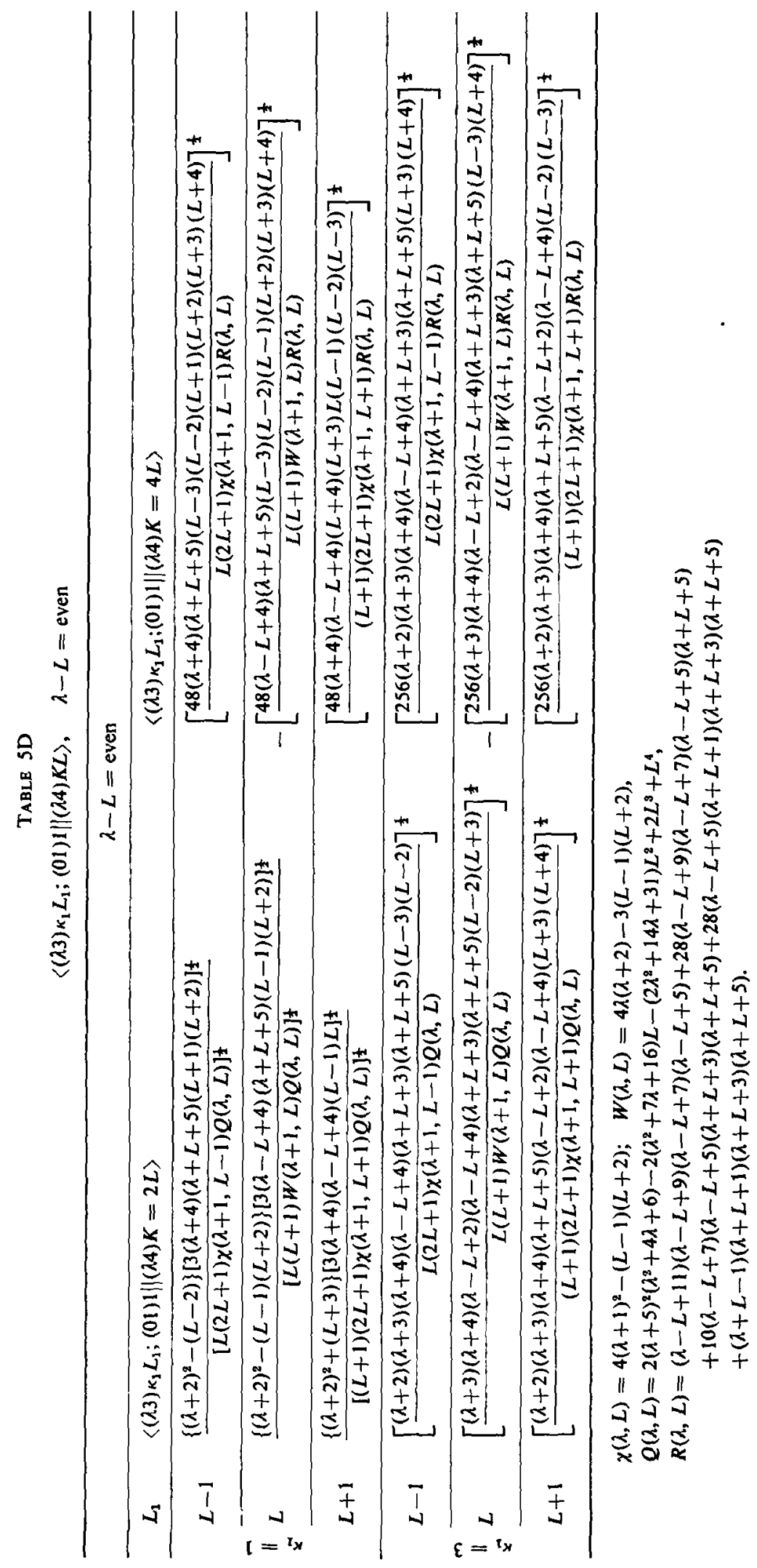


TABLE 5E

$\left\langle(\lambda 3) \kappa_{1} L_{1} ;(01) 1 \|(\lambda 4) \kappa L\right\rangle, \quad \lambda-L=$ even $\quad L \leqq 3$

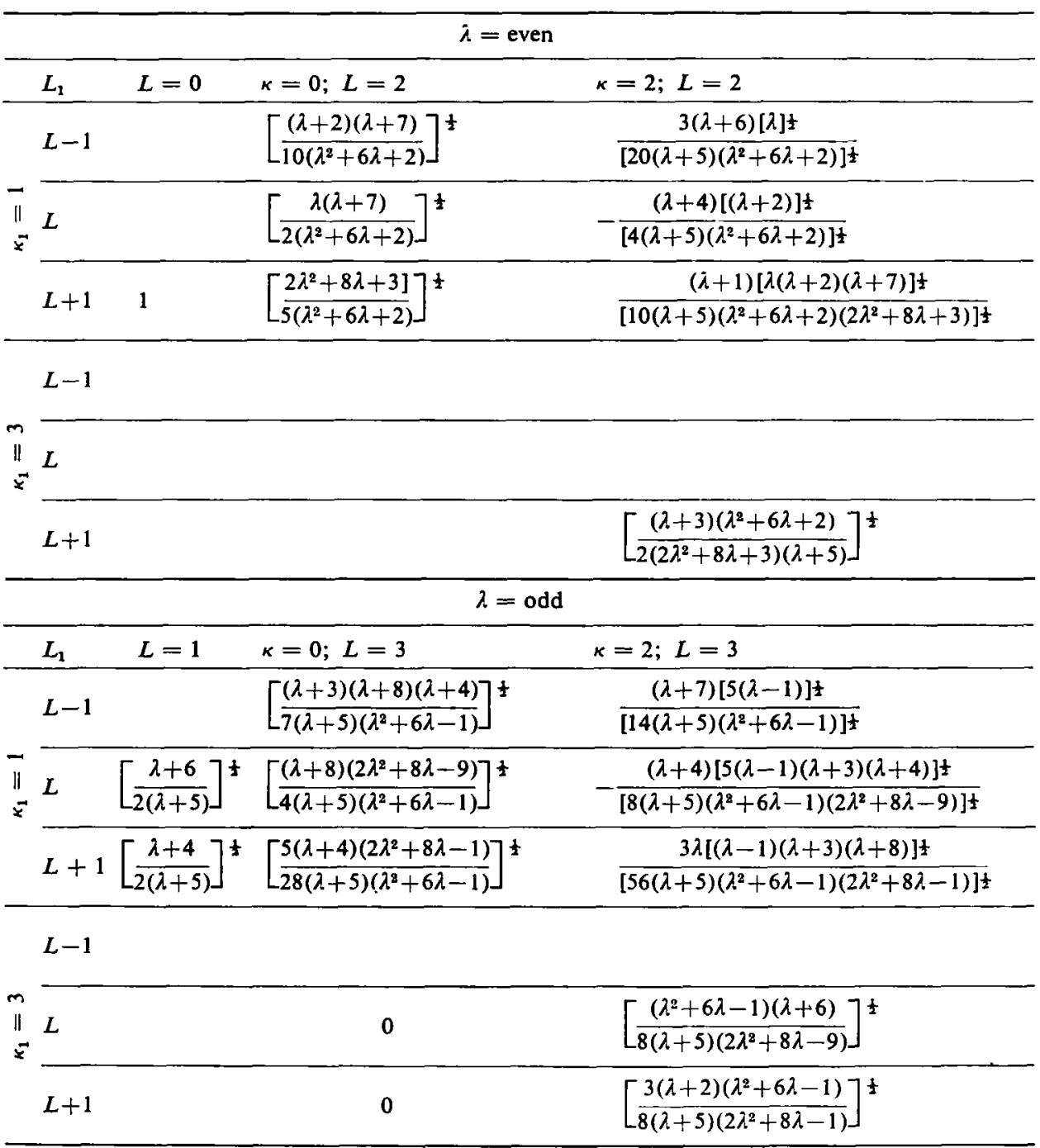




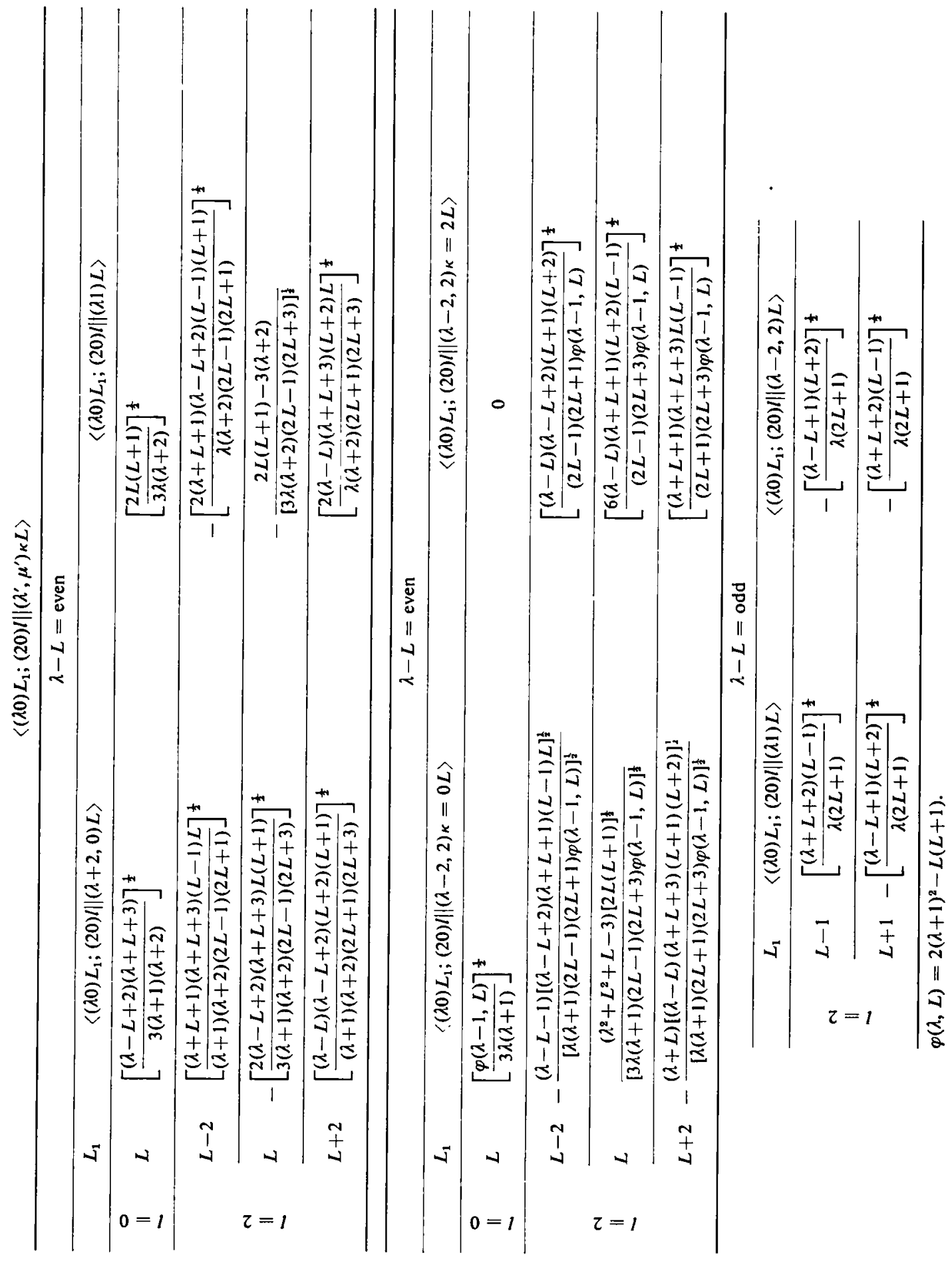




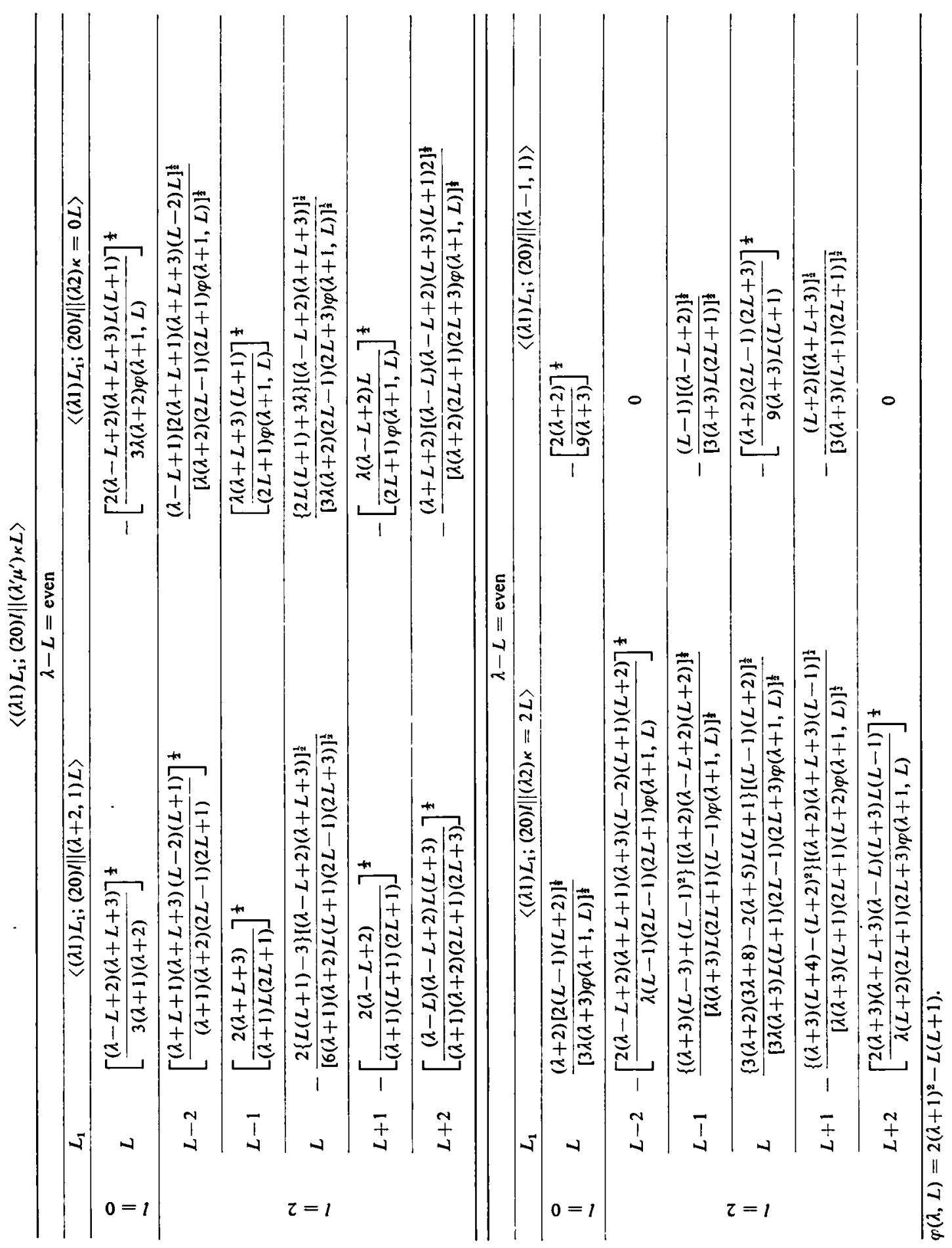




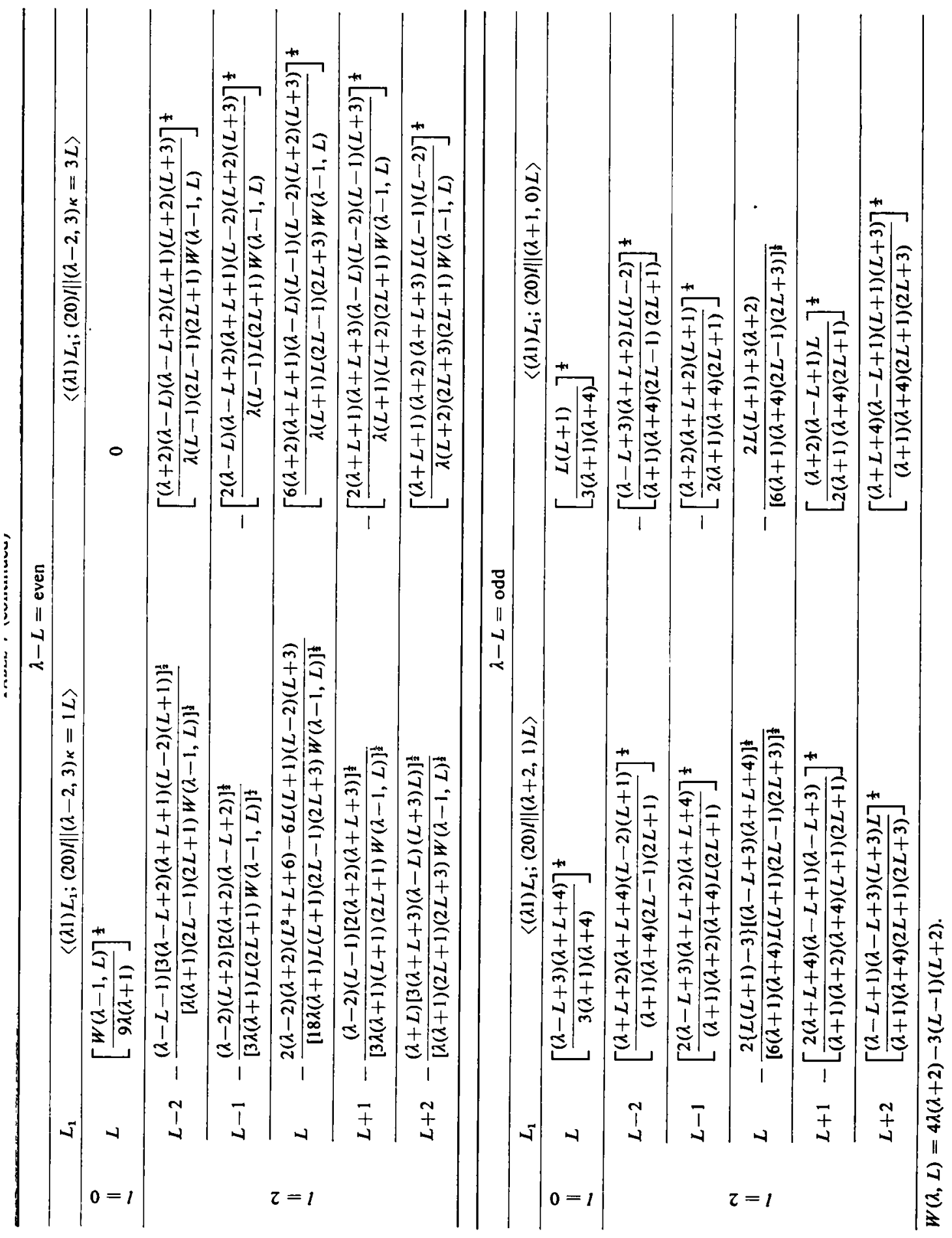




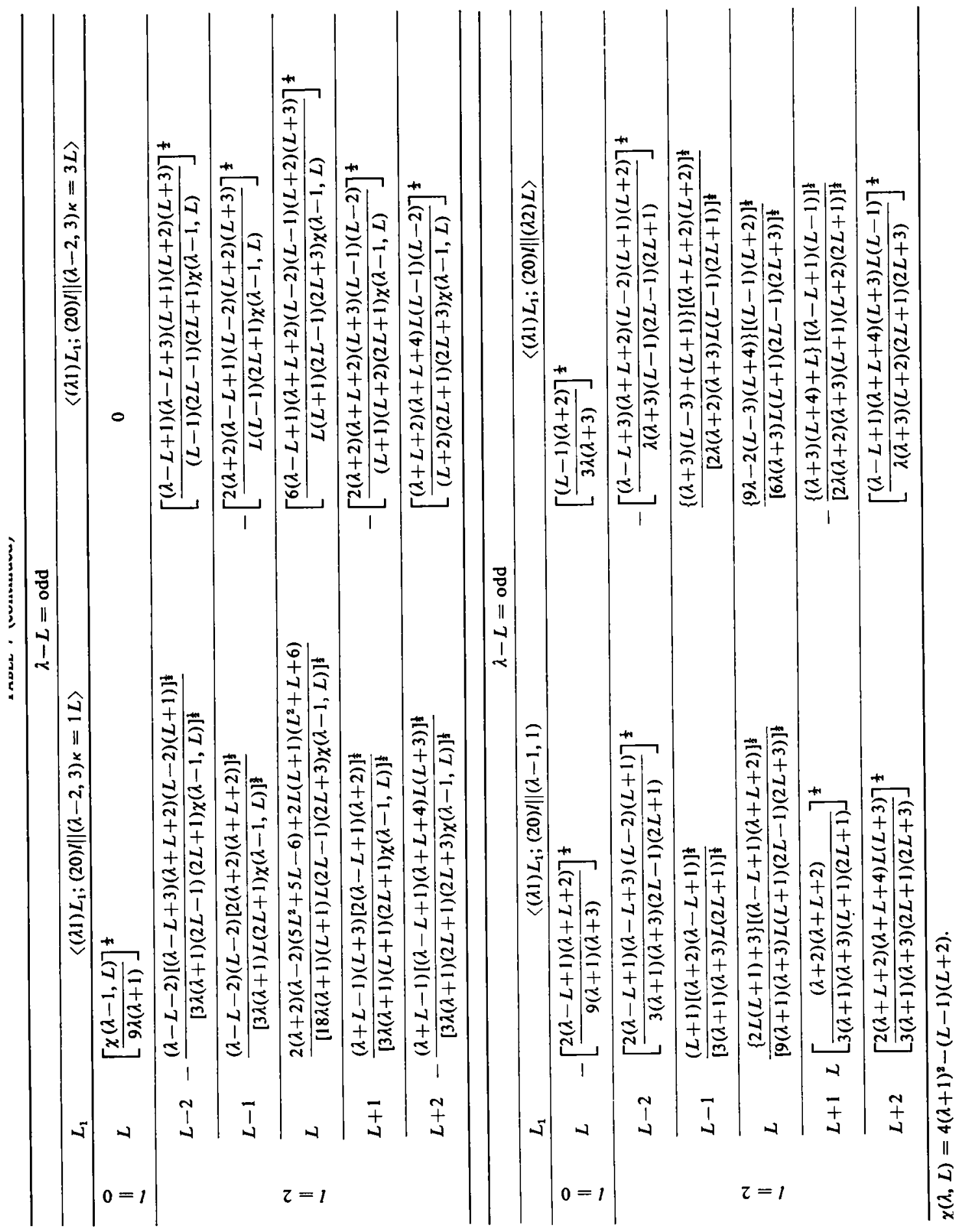




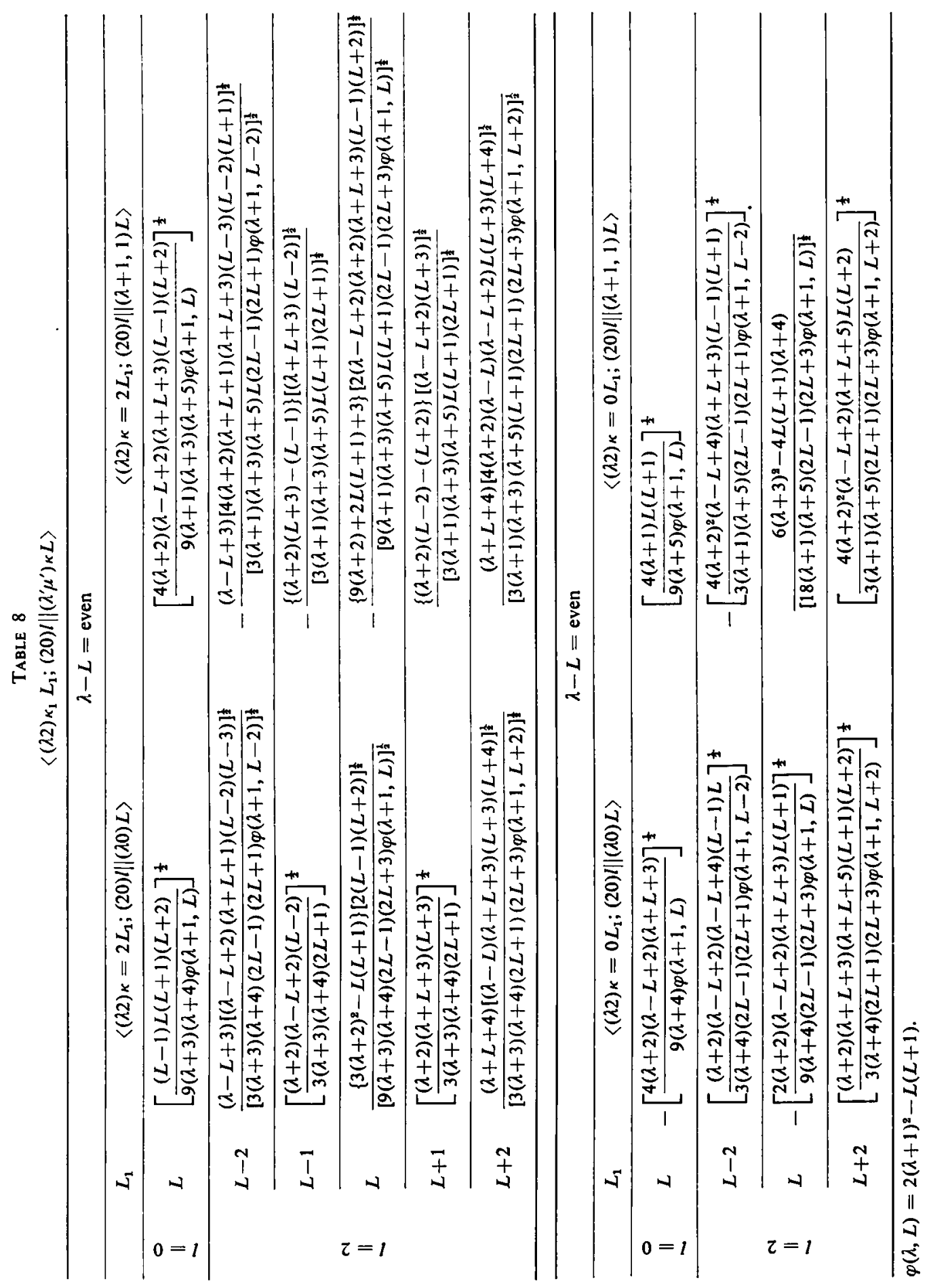




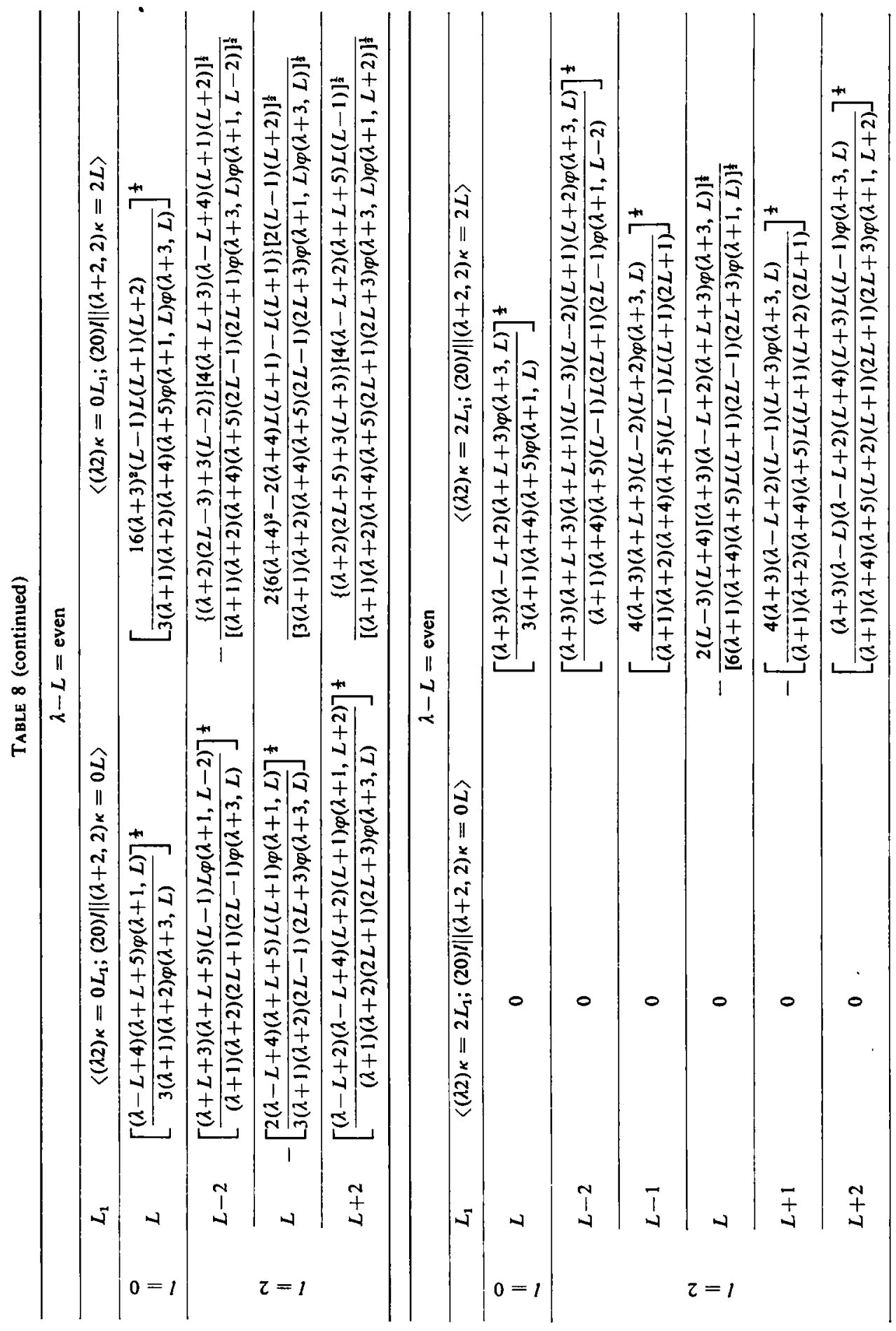


TABLE 8 (continued)

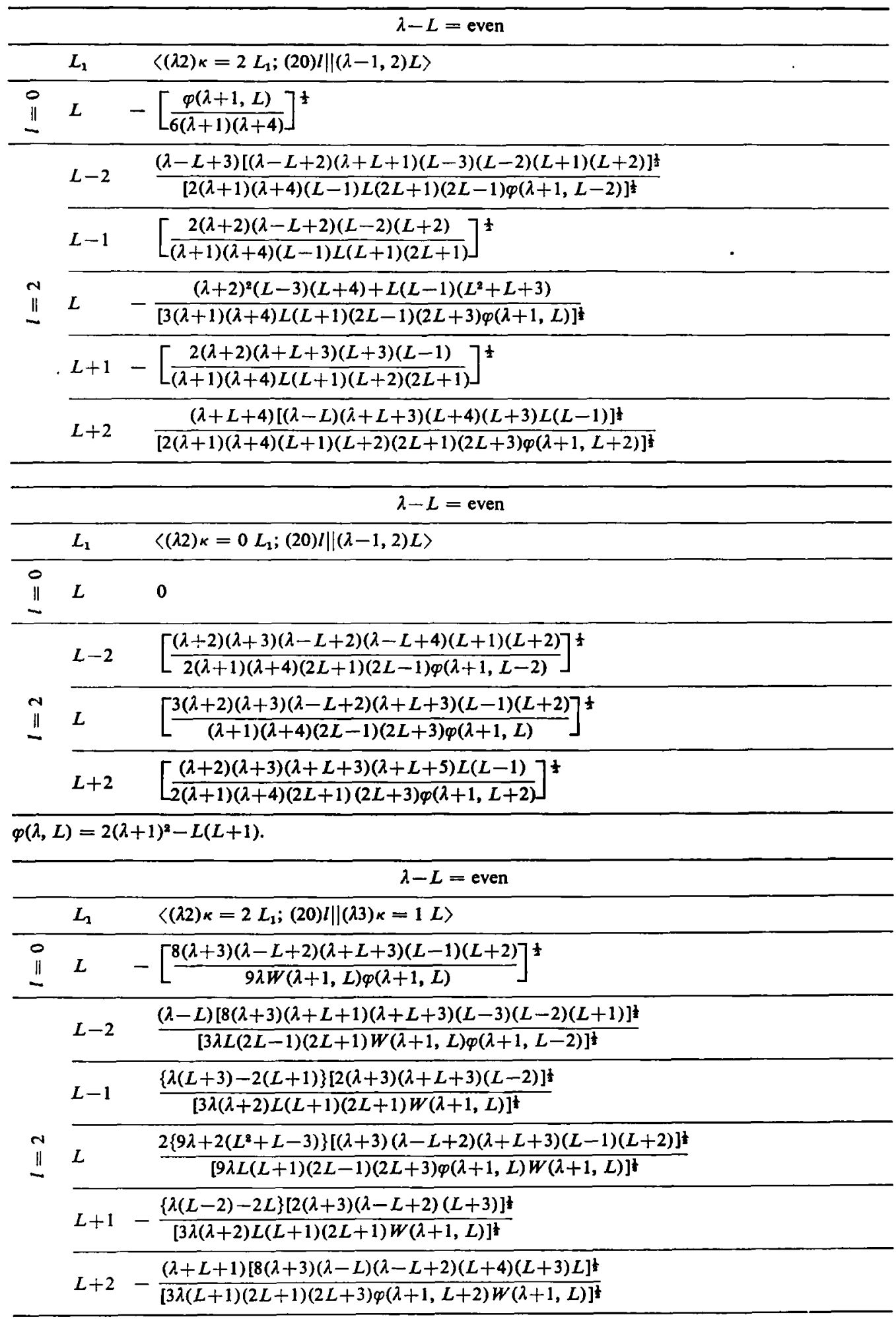


TABLE 8 (continued)

\begin{tabular}{|c|c|c|}
\hline & & $\lambda-L=$ even \\
\hline & $L_{1}$ & $\left\langle(\lambda 2) \kappa=0 L_{1} ;(20) l \|(\lambda 3) \kappa=1 L\right\rangle$ \\
\hline$\stackrel{0}{\sim}$ & $L$ & {$\left[\frac{2 L(L+1) W(\lambda+1, L)}{9 \lambda(\lambda+2) \varphi(\lambda+1, L)}\right]^{\frac{1}{2}}$} \\
\hline \multirow{3}{*}{$\stackrel{\sim}{n}$} & $L-2$ & $-\frac{W(\lambda, L-1)[2(\lambda-L+4)(\lambda+L+3)(L-1)(L+1)]^{\frac{1}{2}}}{[3 \lambda(\lambda+2)(2 L-1)(2 L+1) W(\lambda+1, L) \varphi(\lambda+1, L-2)]^{\frac{1}{3}}}$ \\
\hline & $L$ & $\frac{\left\{12(\lambda+2)^{3}-8(\lambda+2)(\lambda+2) L(L+1)+3(\lambda+2)(L+3)(L-2)-28 L(L+1)+6 L^{2}(L+1)^{2}+48\right\}}{[9 \lambda(\lambda+2)(2 L-1)(2 L+3) \varphi(\lambda+1, L) W(\lambda+1, L)]\}}$ \\
\hline & $L+2$ & $\frac{W(\lambda, L+1)[2(\lambda+L+5)(\lambda-L+2)(L+2) L]^{\frac{1}{2}}}{[3 \lambda(\lambda+2)(2 L+1)(2 L+3) \varphi(\lambda+1, L+2) W(\lambda+1, L)]^{\frac{1}{3}}}$ \\
\hline
\end{tabular}

$\varphi(\lambda, L)=2(\lambda+1)^{2}-L(L+1)$,

$W(\lambda, L)=4 \lambda(\lambda+2)-3(L-1)(L+2)$.

\begin{tabular}{|c|c|c|}
\hline \multicolumn{3}{|r|}{$\lambda-L=$ even } \\
\hline & $L_{1}$ & $\left\langle(\lambda .2)_{\kappa}=2 L_{1} ;(20) l \|(\lambda 3)_{\kappa}=3 L\right\rangle$ \\
\hline$\stackrel{0}{11}$ & $L$ & {$\left[\frac{2(L-2)(L+3) \varphi(\lambda+1, L)}{3 \lambda(\lambda+4) W(\lambda+1, L)}\right]^{\frac{1}{2}}$} \\
\hline \multirow{5}{*}{$\stackrel{\sim}{\sim}$} & $L-2$ & $-\frac{\varphi(\lambda+2, L-1)[2(\lambda-L+2)(\lambda+L+1)(L-3)(L+1)(L+2)(L+3)]^{\frac{1}{2}}}{[\lambda(\lambda+4)(L-1) L(2 L-1)(2 L+1) W(\lambda+1, L) \varphi(\lambda+1, L-2)]^{\frac{1}{2}}}$ \\
\hline & $L-1$ & $\frac{\left\{(\lambda+3)^{2}(L-5)+(\lambda+3)(L-1)(L-2)+(L-1)(L+1)\right\}[2(\lambda-L+2)(L+2)(L+3)]^{\frac{1}{2}}}{[\lambda(\lambda+2)(\lambda+4)(L-1) L(L+1)(2 L+1) W(\lambda+1, L)]^{\frac{\xi}{3}}}$ \\
\hline & $L$ & $\frac{\left\{30(\lambda+2)(\lambda+3)^{2}-6(\lambda+3)(2 \lambda+3)-(\lambda+3)(4 \lambda+31) L(L+1)+\left(2 L^{2}+2 L+3\right)(L-1)(L+2)\right\}[(L-2)(L+3)]^{\ddagger}}{[3 \lambda(\lambda+4) L(L+1)(2 L-1)(2 L+3) \varphi(\lambda+1, L) W(\lambda+1, L)]^{\ddagger}}$ \\
\hline & $L+1$ & $-\frac{\left\{(\lambda+3)^{2}(L+6)-L(L+2)-(\lambda+3)(L+2)(L+3)\right\}[2(\lambda+L+3)(L-1)(L-2)]^{\frac{1}{2}}}{[\lambda(\lambda+2)(\lambda+4) L(L+1)(L+2)(2 L+1) W(\lambda+1, L)]^{t}}$ \\
\hline & $L+2$ & $\frac{\varphi(\lambda+2, L+1)[2(\lambda+L+3)(\lambda-L)(L+4) L(L-1)(L-2)]^{\dagger}}{[\lambda(\lambda+4)(L+2)(L+1)(2 L+3)(2 L+1) W(\lambda+1, L) \varphi(\lambda+1, L+2)]^{\frac{1}{z}}}$ \\
\hline
\end{tabular}

$$
\lambda-L=\text { even }
$$

\begin{tabular}{lll}
\hline & $L_{1}$ & $\left\langle(\lambda 2) \kappa=0 L_{1} ;(20) l \|(\lambda .3) \kappa=3 L\right\rangle$ \\
\hline 0 & $L$ & 0 \\
\hline & $L-2$ & {$\left[\frac{8(\lambda+3)(\lambda-L+2)(\lambda-L+4)(L-2)(L+1)(L+2)(L+3)}{\lambda(\lambda+2)(\lambda+4)(2 L-1)(2 L+1) W(\lambda+1, L) \varphi(\lambda+1, L-2)]}\right]^{\frac{1}{2}}$} \\
\cline { 2 - 2 } & $L$ & {$\left[\frac{48(\lambda+3)(\lambda-L+2)(\lambda+L+3)(L-2)(L-1)(L+2)(L+3)}{\lambda(\lambda+2)(\lambda+4)(2 L-1)(2 L+3) \varphi(\lambda+1, L) W(\lambda+1, L)]}\right]^{\frac{1}{2}}$} \\
\cline { 2 - 2 } & $L+2$ & {$\left[\frac{8(\lambda+3)(\lambda+L+3)(\lambda+L+5)(L+3) L(L-1)(L-2)}{\lambda(\lambda+2)(\lambda+4)(2 L+1)(2 L+3) W(\lambda+1, L) \varphi(\lambda+1, L+2)}\right]^{\frac{1}{2}}$}
\end{tabular}

$\varphi(\lambda, L)=2(\lambda+1)^{2}-L(L+1)$,

$W(\lambda, L)=4 \lambda(\lambda+2)-3(L-1)(L+2)$. 


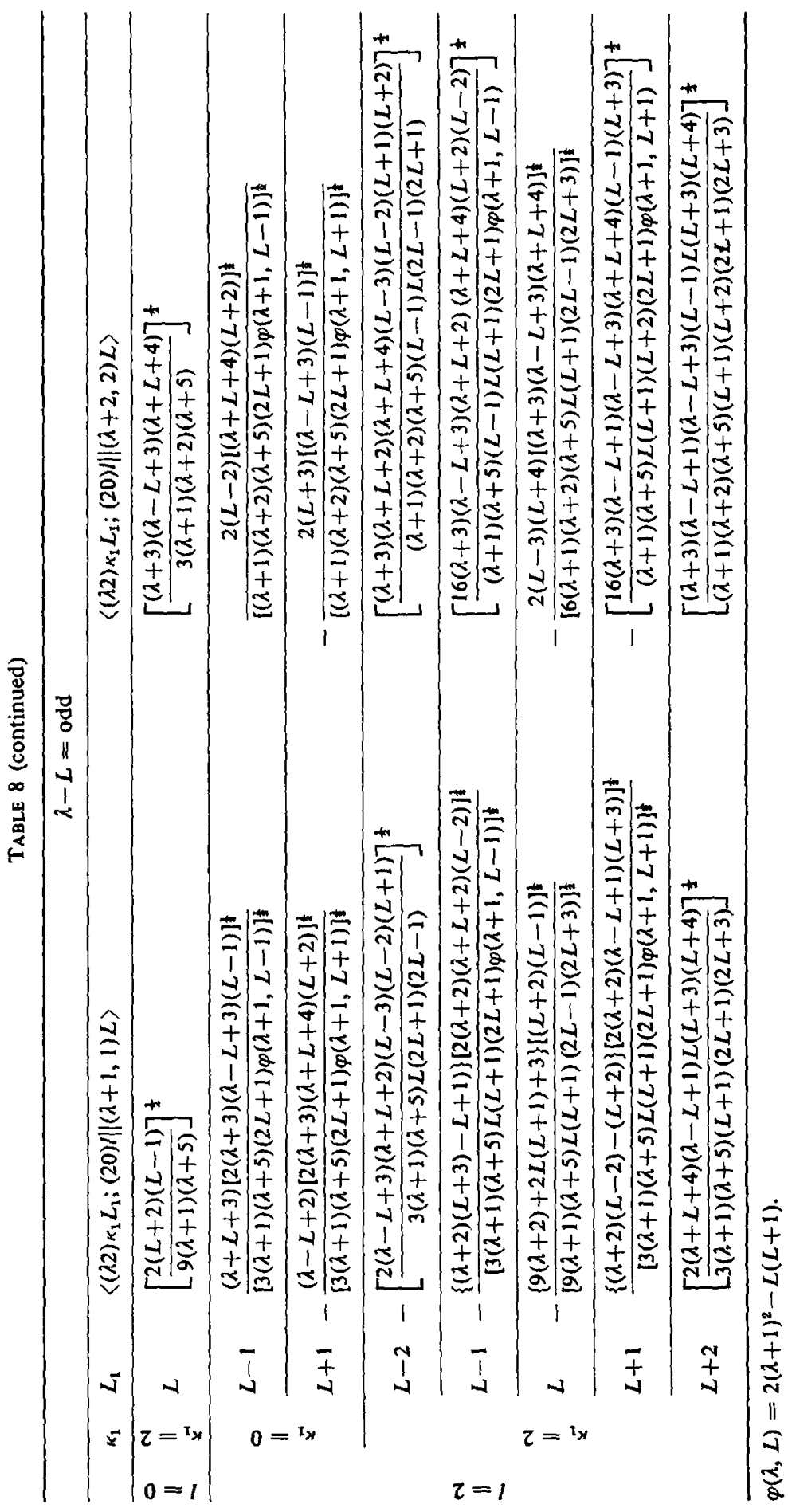




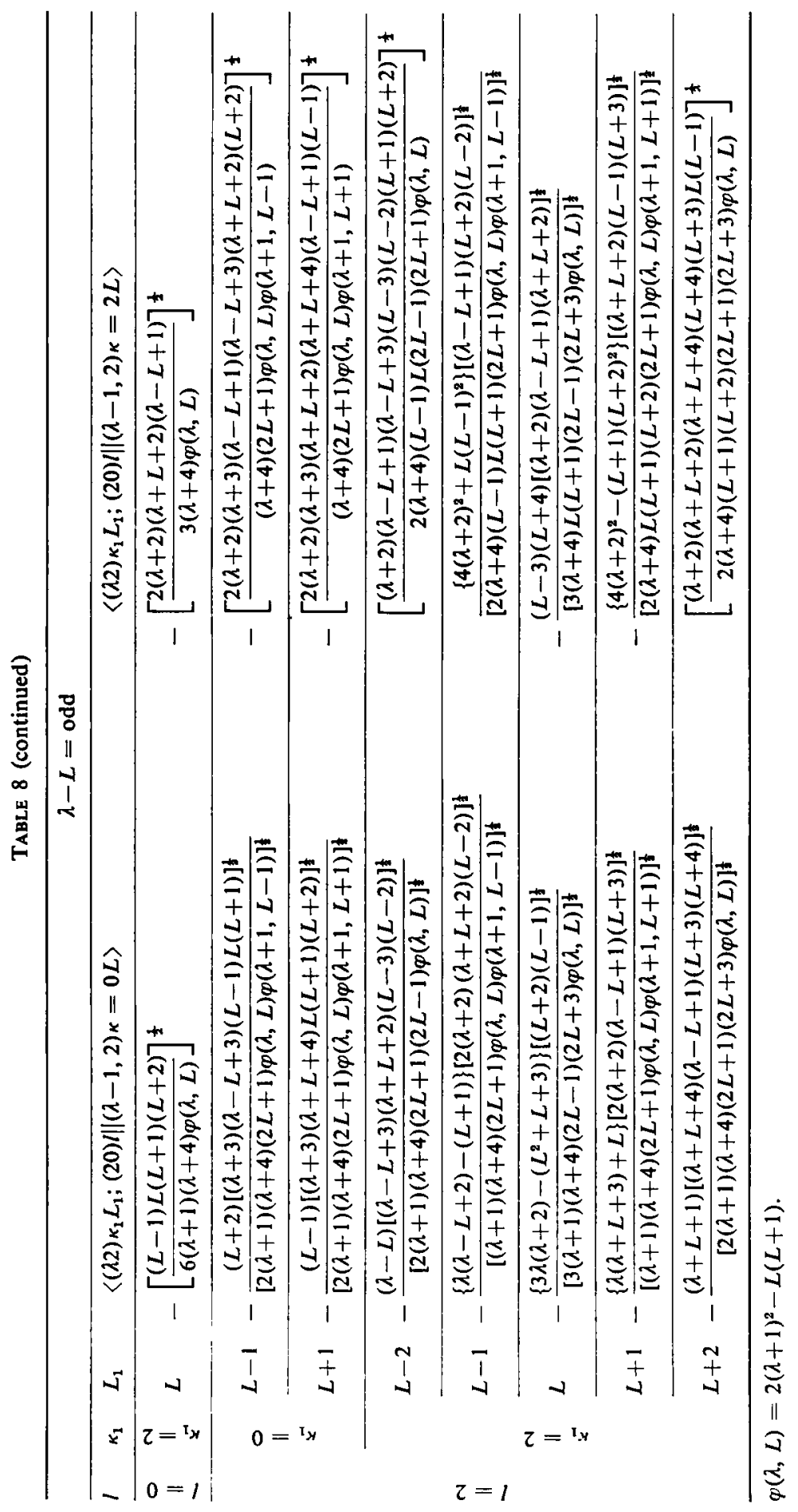


Table 8 (continued)

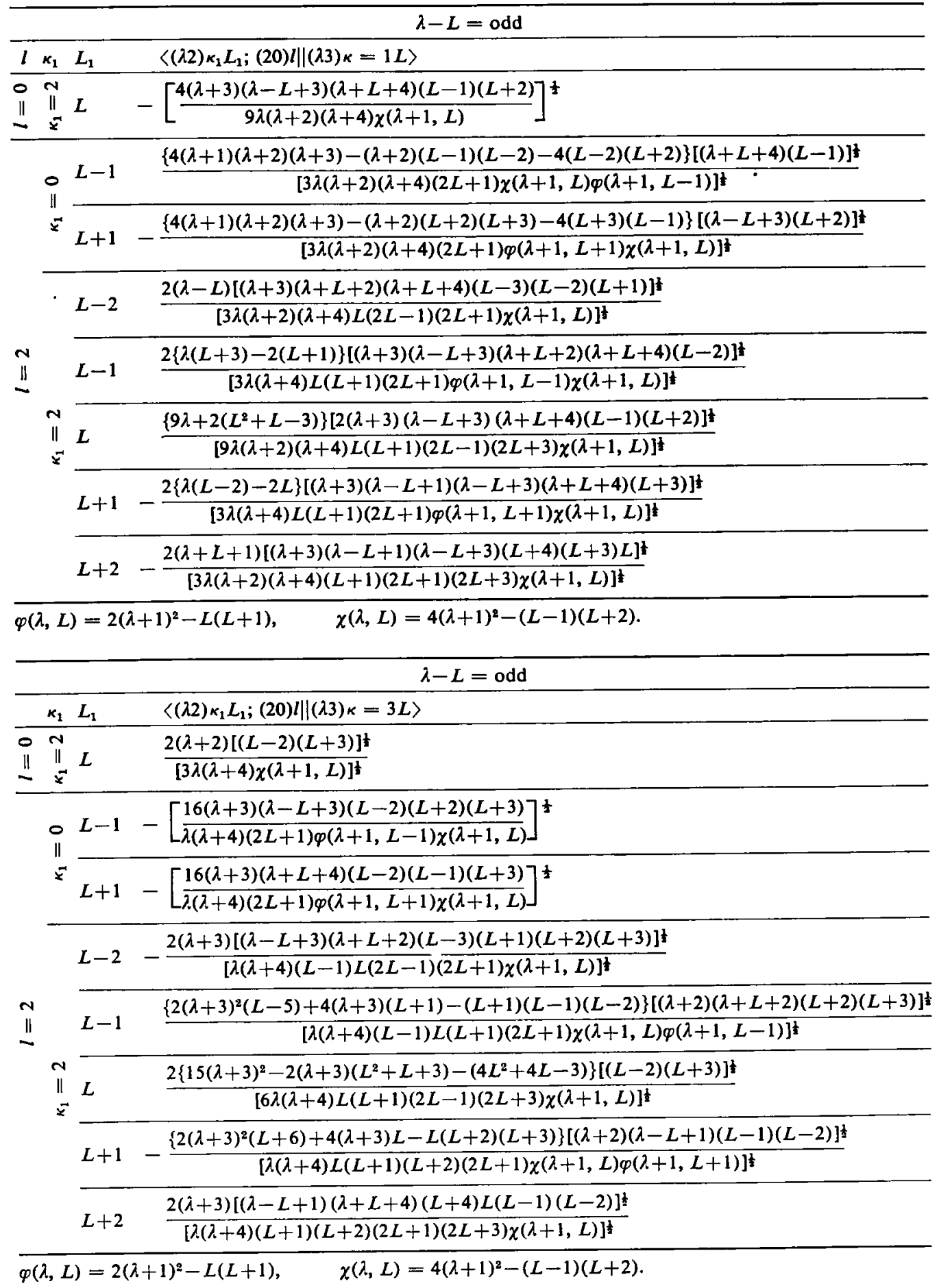




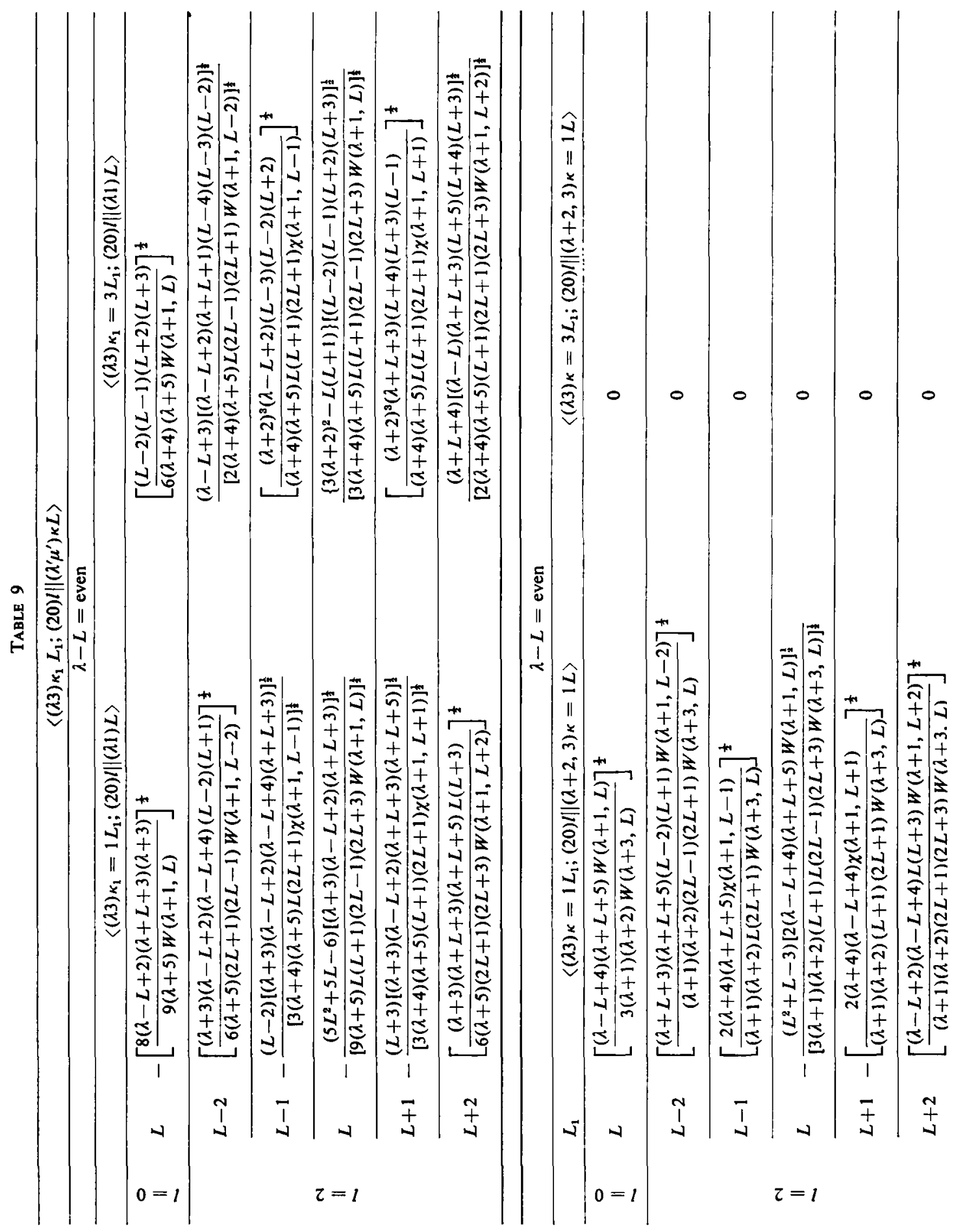


Table 9 (continued)

\begin{tabular}{|c|c|}
\hline & $\lambda-L=$ even \\
\hline & $\left\langle(\lambda .3) \kappa=1 L_{1} ;(20) l \|(\lambda+1,2) L\right\rangle$ \\
\hline il & $L \quad \lambda\left[\frac{(L-1)(L+2)}{3(\lambda+1)(\lambda+6) W(\lambda+1, L)}\right]^{\frac{1}{2}}$ \\
\hline \multirow{5}{*}{$\stackrel{-1}{11}$} & $L-2-\frac{(\lambda+3)[(\lambda-L+4)(\lambda+L+3)(L-2)(L+1)(L+2)]^{\frac{1}{2}}}{[(\lambda+1)(\lambda+6)(L-1)(2 L-1)(2 L+1) W(\lambda+1, L-2)]^{\frac{1}{2}}}$ \\
\hline & $L-1 \quad \frac{\left\{(\lambda+3)^{2}(L-3)+(\lambda+3)(L+1)(L-2)-(L-1)(L-3)\right\}[(\lambda-L+4)(L+2)]^{\frac{1}{2}}}{[2(\lambda+1)(\lambda+4)(\lambda+6) L(L-1)(2 L+1) \chi(\lambda+1, L-1)]^{\frac{1}{2}}}$ \\
\hline & $L \quad \frac{\left\{9(\lambda+3)^{2}+6(\lambda+3)-2(\lambda+9) L(L+1)+9\right\}[(L-1)(L+2)]^{\frac{1}{2}}}{[6(\lambda+1)(\lambda+6) L(L+1)(2 L-1)(2 L+3) W(\lambda+1, L)]^{\frac{1}{2}}}$ \\
\hline & $L+1-\frac{\left\{(\lambda+3)^{2}(L+4)-(\lambda+3) L(L+3)+(L+2)(L+4)\right\}[(\lambda+L+5)(L-1)]^{\mid}}{[2(\lambda+1)(\lambda+4)(\lambda+6)(L+1)(L+2)(2 L+1) \chi(\lambda+1, L+1)]^{\frac{1}{2}}}$ \\
\hline & $L+2 \quad \frac{(\lambda+3)[(\lambda-L+2)(\lambda+L+5)(L-1) L(L+3)]^{\frac{1}{2}}}{[(\lambda+1)(\lambda+6)(L+2)(2 L+1)(2 L+3) W(\lambda+1, L+2)]^{\frac{1}{2}}}$ \\
\hline \multicolumn{2}{|r|}{$\lambda-L=$ even } \\
\hline & $\left\langle(\lambda 3) \kappa=3 L_{1} ;(20) / \|(\lambda+1,2) L\right\rangle$ \\
\hline i & {$\left[\frac{(\lambda+3)(\lambda-L+2)(\lambda+L+3)(L-2)(L+3)}{(\lambda+1)(\lambda+4)(\lambda+6) W(\lambda+1, L)}\right]^{\frac{1}{2}}$} \\
\hline \multirow{5}{*}{$\stackrel{N}{\sim}$} & $L-2-\frac{(\lambda-L+3)[3(\lambda+3)(\lambda+L+1)(\lambda+L+3)(L-4)(L-3)(L-2)(L+2)]^{1}}{[(\lambda+6)(\lambda+1)(\lambda+4)(L-1) L(2 L-1)(2 L+1) W(\lambda+1, L-2)]^{\mid}}$ \\
\hline & $L-1-\frac{\{(\lambda+2)(L+5)-2(L-1)\}[3(\lambda+2)(\lambda+L+3)(\lambda+3)(L-3)(L-2)]^{\ddagger}}{[2(\lambda+1)(\lambda+4)(\lambda+6)(L-1) L(L+1)(2 L+1) \chi(\lambda+1, L-1)]^{\mid}}$ \\
\hline & $-\frac{\{15(\lambda+2)+2 L(L+1)+6\}[(\lambda+3)(\lambda-L+2)(\lambda+L+3)(L-2)(L+3)]}{[2(\lambda+1)(\lambda+4)(\lambda+6) L(L+1)(2 L-1)(2 L+3) W(\lambda+1, L)]^{\frac{1}{3}}}$ \\
\hline & $\frac{\{(\lambda+2)(L-4)-2(L+2)\}[3(\lambda+2)(\lambda+3)(\lambda-L+2)(L+4)(L+3)]^{\frac{1}{3}}}{[2(\lambda+1)(\lambda+4)(\lambda+6) L(L+1)(L+2)(2 L+1) \chi(\lambda+1, L+1)]^{\frac{1}{3}}}$ \\
\hline & $L+2 \quad \frac{(\lambda+L+4)[3(\lambda+3)(\lambda-L)(\lambda-L+2)(L+5)(L+4)(L+3)(L-1)]^{t}}{[(\lambda+1)(\lambda+4)(\lambda+6)(L+1)(L+2)(2 L+1)(2 L+3) W(\lambda+1, L+2)]^{t}}$ \\
\hline
\end{tabular}


TABLE 9 (continued)

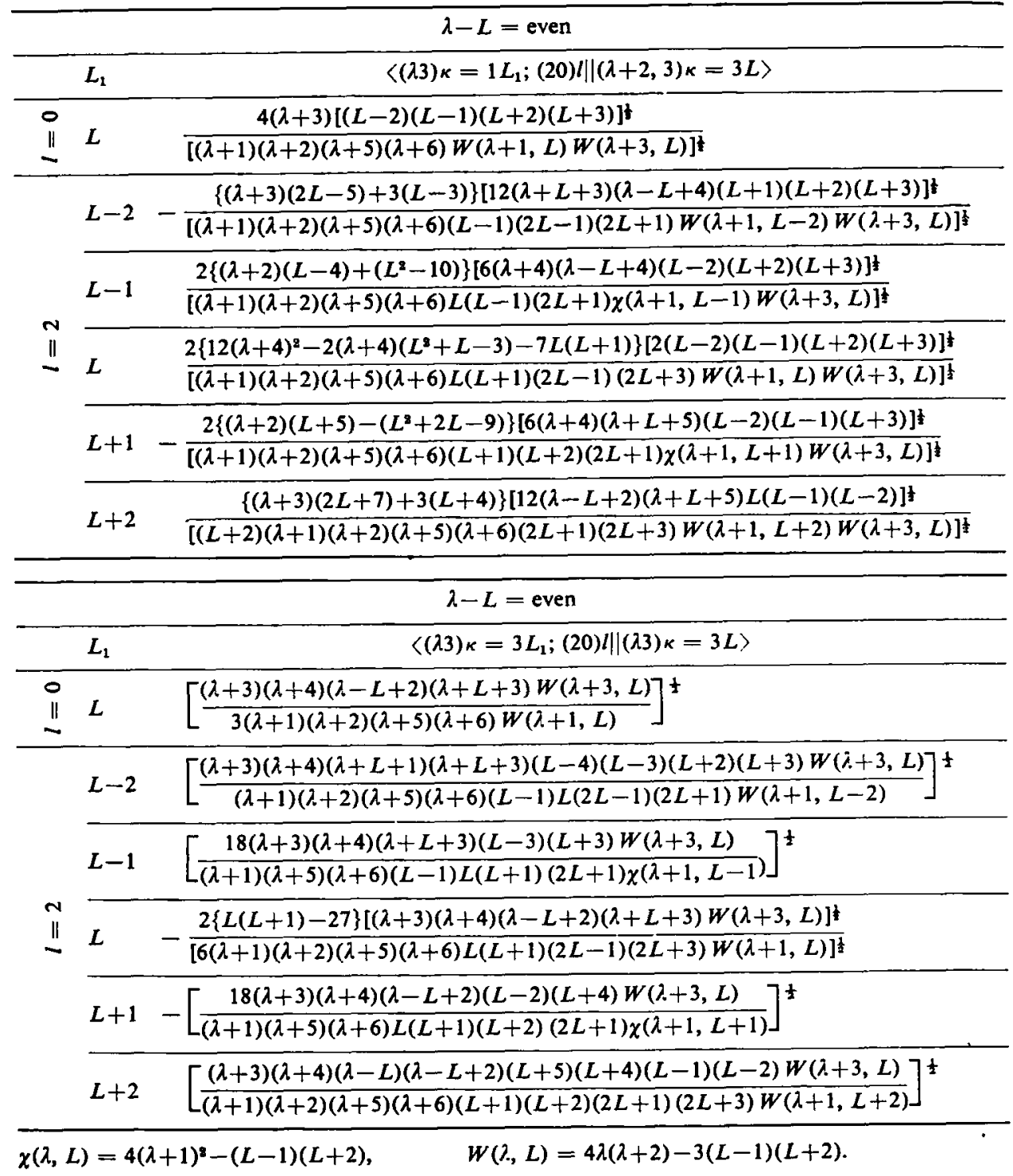


TABle 9 (continued)

\begin{tabular}{|c|c|c|}
\hline & & $\lambda-L=$ even \\
\hline & $L_{1}$ & $\left\langle(\lambda 3) \kappa=1 L_{1} ;(20) l \|(\lambda-1,3) \kappa=1 L\right\rangle$ \\
\hline II & $L$ & $-\left[\frac{2(\lambda+3)(\lambda+4) \chi(\lambda, L)}{45(\lambda+1)(\lambda+5) W(\lambda+1, L)}\right]^{\frac{1}{2}}$ \\
\hline \multirow{5}{*}{$\stackrel{n}{\sim}$} & $L-2$ & $-\frac{(\lambda-L-1)[8(\lambda+3)(\lambda+4)(\lambda-L+4)(\lambda+L+3)(L-2)(L+1)]^{ \pm}}{[15(\lambda+1)(\lambda+5)(2 L-1)(2 L+1) \chi(\lambda, L) W(\lambda+1, L-2)]^{\mathbf{t}}}$ \\
\hline & $L-1$ & $-\frac{\left\{4(\lambda+1)^{2}(2 L-1)+\left(L^{3}+8 L^{2}-7 L-38\right)\right\}[(\lambda+3)(\lambda-L+4)]^{\ddagger}}{[15(\lambda+1)(\lambda+5) L(2 L+1) \chi(\lambda, L) \chi(\lambda+1, L-1)]^{\ddagger}}$ \\
\hline & $L$ & $-\frac{\left\{4(\lambda+1)^{2}\left(7 L^{2}+7 L-3\right)-(L-1)(L+2)\left(16 L^{2}+16 L-57\right)\right\}[(\lambda+3)(\lambda+4)]^{\ddagger}}{[45(\lambda+1)(\lambda+5) L(L+1)(2 L-1)(2 L+3) W(\lambda+1, L) \chi(\lambda, L)]^{\frac{1}{3}}}$ \\
\hline & $L+1$ & $-\frac{\left\{4(\lambda+1)^{2}(2 L+3)+\left(L^{3}-5 L^{2}-20 L+24\right)\right\}[(\lambda+3)(\lambda+L+5)]^{\ddagger}}{[15(\lambda+1)(\lambda+5)(L+1)(2 L+1) \chi(\lambda, L) \chi(\lambda+1, L+1)]^{\ddagger}}$ \\
\hline & $L+2$ & $-\frac{(\lambda+L)[8(\lambda+3)(\lambda+4)(\lambda-L+2)(\lambda+L+5) L(L+3)]^{1}}{[15(\lambda+1)(\lambda+5)(2 L+1)(2 L+3) W(\lambda+1, L+2) \chi(\lambda, L)]^{\frac{1}{2}}}$ \\
\hline
\end{tabular}

\begin{tabular}{|c|c|c|}
\hline & & $\lambda-L=$ even \\
\hline & $L_{1}$ & $\left\langle(\lambda 3)_{\kappa}=3 L_{1} ;(20) l \|(\lambda-1,3) \kappa=1 L\right\rangle$ \\
\hline il & $L$ & $-\left[\frac{8(\lambda-L+2)(\lambda+L+3)(L-2)(L-1)(L+2)(L+3)}{15(\lambda+1)(\lambda+5) W(\lambda+1, L) \chi(\lambda, L)}\right]^{\frac{1}{2}}$ \\
\hline \multirow{5}{*}{$\stackrel{N}{\sim}$} & $L-2$ & $-\frac{(\lambda-L-1)(\lambda-L+3)[8(\lambda+L+1)(\lambda+L+3)(L-4)(L-3)(L-2)]^{\frac{1}{3}}}{[5(\lambda+1)(\lambda+5) L(2 L-1)(2 L+1) W(\lambda+1, L-2) \chi(\lambda, L)]^{\frac{3}{3}}}$ \\
\hline & $L-1$ & $-\frac{2\{2(\lambda+2)(\lambda-L-1)+3(L-1)\}[(\lambda+2)(\lambda+L+3)(L-2)(L-3)(L+2)]^{\sharp}}{[5(\lambda+1)(\lambda+5) L(L+1)(2 L+1) \chi(\lambda+1, L-1) \chi(\lambda, L)]^{\frac{1}{3}}}$ \\
\hline & $L$ & $-\frac{2\{6(\lambda+2)(\lambda-1)-2 L(L+1)-9\}[(\lambda-L+2)(\lambda+L+3)(L+3)(L-2)(L+2)(L-1)]^{1}}{[15(\lambda+1)(\lambda+5) L(L+1)(2 L-1)(2 L+3) \chi(\lambda, L) W(\lambda+1, L)]^{\ddagger}}$ \\
\hline & $L+1$ & $-\frac{2\{2(\lambda+2)(\lambda+L)-3(L+2)\}[(\lambda+2)(\lambda-L+2)(L-1)(L+3)(L+4)]^{\ddagger}}{[5(\lambda+1)(\lambda+5) L(L+1)(2 L+1) \chi(\lambda, L) \chi(\lambda+1, L+1)]^{\frac{1}{2}}}$ \\
\hline & $L+2$ & $-\frac{(\lambda+L)(\lambda+L+4)[8(\lambda-L)(\lambda-L+2)(L+3)(L+4)(L+5)]^{\frac{1}{2}}}{[5(\lambda+1)(\lambda+5)(L+1)(2 L+1)(2 L+3) W(\lambda+1, L+2) \chi(\lambda, L)]^{\mid}}$ \\
\hline
\end{tabular}

$\chi(\lambda, L)=4(\lambda+1)^{2}-(L-1)(L+2)$,

$W(\lambda, L)=4 \lambda(\lambda+2)-3(L-1)(L+2)$. 
TABLE 9 (continued)

\begin{tabular}{|c|c|c|}
\hline & & $\lambda-L=$ even \\
\hline & $L_{1}$ & $\left\langle(\lambda 3)^{\prime} \kappa=1 L_{1} ;(20) l \|(\lambda-1,3) \kappa=3 L\right\rangle$ \\
\hline$\stackrel{0}{11}$ & $L$ & 0 \\
\hline & $L-2$ & {$\left[\frac{8(\lambda+2)(\lambda+3)(\lambda+4)(\lambda-L+2)(\lambda-L+4)(L+1)(L+2)(L+3)}{5(\lambda+5)(L-1)(2 L-1)(2 L+1) W(\lambda+1, L-2) \chi(\lambda, L)}\right]^{\frac{1}{2}}$} \\
\hline & $L-1$ & $-\left[\frac{16(\lambda+2)(\lambda+3)(\lambda-L+2)(\lambda-L+4)(\lambda+L+3)(L-2)(L+2)(L+3)}{5(\lambda+5)(L-1) L(2 L+1) \chi(\lambda+1, L-1) \chi(\lambda, L)}\right]^{\frac{1}{2}}$ \\
\hline$\stackrel{11}{n}$ & $L$ & {$\left[\frac{48(\lambda+2)(\lambda+3)(\lambda+4)(\lambda-L+2)(\lambda+L+3)(L-2)(L-1)(L+2)(L+3)}{5(\lambda+5) L(L+1)(2 L-1)(2 L+3) W(\lambda+1, L) \chi(\lambda, L)}\right]^{\frac{1}{2}}$} \\
\hline & $L+1$ & $-\left[\frac{16(\lambda+2)(\lambda+3)(\lambda+L+3)(\lambda+L+5)(\lambda-L+2)(L+3)(L-1)(L-2)}{5(\lambda+5)(L+1)(L+2)(2 L+1) \chi(\lambda+1, L+1) \chi(\lambda, L)}\right]^{\frac{1}{2}}$ \\
\hline & $L+2$ & {$\left[\frac{8(\lambda+2)(\lambda+3)(\lambda+4)(\lambda+L+3)(\lambda+L+5) L(L-1)(L-2)}{5(\lambda+5)(L+2)(2 L+1)(2 L+3) W(\lambda+1, L+2) \chi(\lambda, L)}\right]^{\frac{1}{2}}$} \\
\hline & & $\lambda-L=$ even \\
\hline & $L_{1}$ & $\left\langle(\lambda 3) \kappa=3 L_{1} ;(20) l||(\lambda-1,3) \kappa=3 L\right\rangle$ \\
\hline$\stackrel{0}{\|}$ & $L$ & $-\left[\frac{2(\lambda+2) W(\lambda+1, L)}{5(\lambda+5) \chi(\lambda, L)}\right]^{\frac{1}{2}}$ \\
\hline \multirow{5}{*}{$\stackrel{N}{\|}$} & $L-2$ & $\frac{(\lambda-L+3)[24(\lambda+2)(\lambda-L+2)(\lambda+L+1)(L-4)(L-3)(L+2)(L+3)]^{\frac{1}{2}}}{[5(\lambda+5)(L-1) L(2 L+1)(2 L-1) W(\lambda+1, L-2) \chi(\lambda, L)]^{\frac{1}{3}}}$ \\
\hline & $\boldsymbol{L}-1$ & $\frac{\left\{12(\lambda+2)^{2}+(L-2)(L-1)(L+1)\right\}[3(\lambda-L+2)(L-3)(L+3)]^{\ddagger}}{[5(\lambda+5)(L-1) L(L+1)(2 L+1) \chi(\lambda+1, L-1) \chi(\lambda, L)]^{t}}$ \\
\hline & $L$ & $-\frac{\left\{4(\lambda+2)^{2}\left(L^{2}+L-27\right)+59 L(L+1)-18\right\}[(\lambda+2)]^{\frac{1}{2}}}{[5(\lambda+5) L(L+1)(2 L+3)(2 L-1) W(\lambda+1, L) \chi(\lambda, L)]^{\frac{1}{3}}}$ \\
\hline & $L+1$ & $-\frac{\left\{12(\lambda+2)^{2}-(L+3)(L+2) L\right\}[3(\lambda+L+3)(L-2)(L+4)]^{\}}}{[5(\lambda+5) L(L+1)(L+2)(2 L+1) \chi(\lambda+1, L+1) \chi(\lambda, L)]^{\mathbf{z}}}$ \\
\hline & $L+2$ & $\frac{(\lambda+L+4)[24(\lambda+2)(\lambda-L)(\lambda+L+3)(L+5)(L+4)(L-1)(L-2)]^{\frac{1}{2}}}{[5(\lambda+5)(L+1)(L+2)(2 L+1)(2 L+3) \chi(\lambda, L) W(\lambda+1, L+2)]^{\frac{1}{3}}}$ \\
\hline
\end{tabular}

$\chi(\lambda, L)=4(\lambda+1)^{2}-(L-1)(L+2), \quad W(\lambda, L)=4 \lambda(\lambda+2)-3(L-1)(L+2)$. 


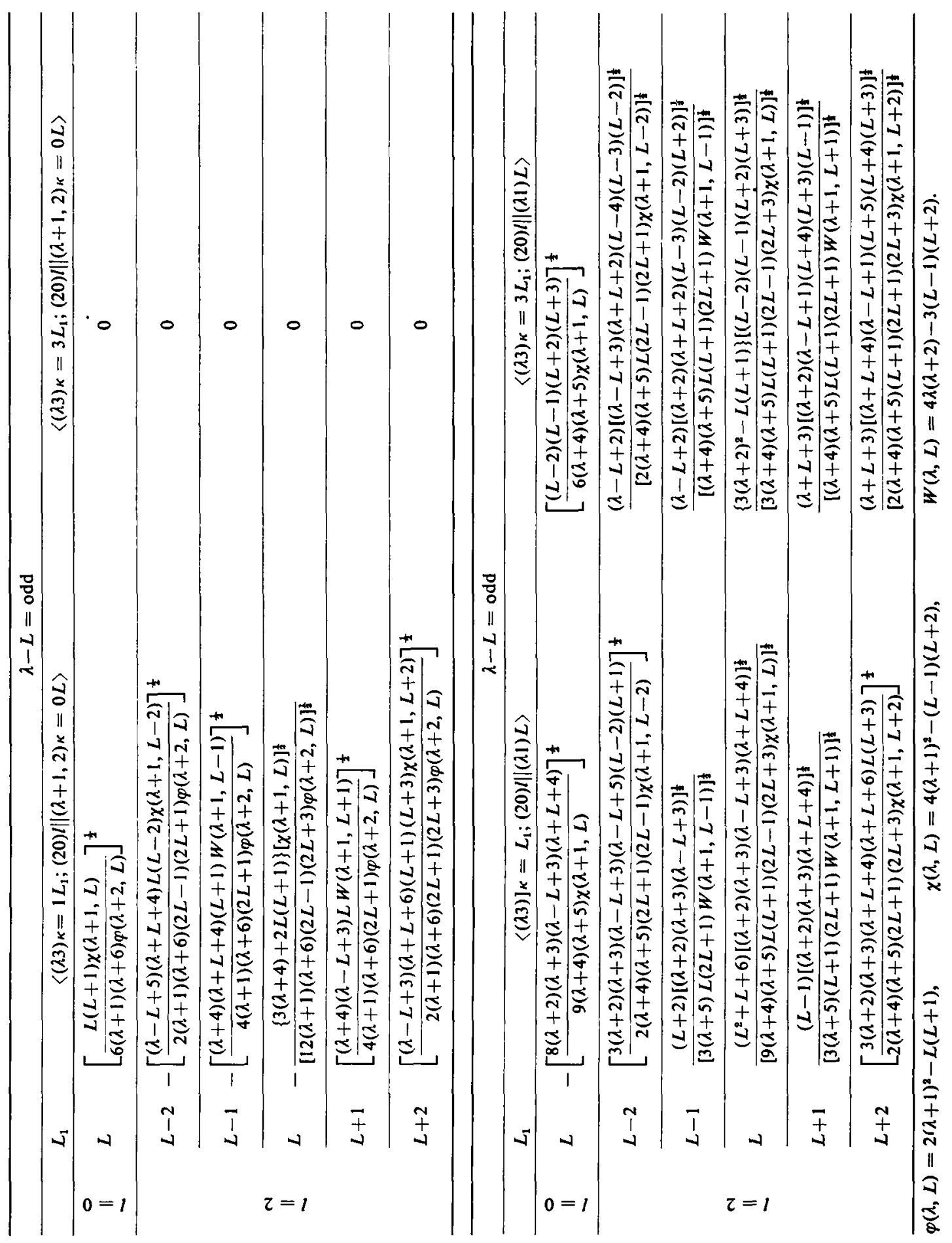


TABLE 9 (continued)

\begin{tabular}{|c|c|c|}
\hline & & $\lambda-L=$ odd \\
\hline & $L_{1}$ & $\left.\left\langle(\lambda 3) \kappa=1 L_{1} ;(20) l \|\right\}(\lambda+1,2) \kappa=2 L\right\rangle$ \\
\hline$\stackrel{0}{\prime \prime}$ & $L$ & $\frac{\lambda[2(\lambda+3)(\lambda-L+3)(\lambda+L+4)(L-1)(L+2)]^{\ddagger}}{[3(\lambda+1)(\lambda+4)(\lambda+6) \varphi(\lambda+2, L) \chi(\lambda+1, L)]^{\frac{1}{2}}}$ \\
\hline & $L-2$ & $-\frac{\left\{(\lambda+3)^{2}+(\lambda+1) L+2\right\}[2(\lambda+3)(\lambda-L+3)(\lambda-L+5)(L-2)(L+1)(L+2)]^{!}}{[(\lambda+1)(\lambda+4)(\lambda+6)(L-1)(2 L-1)(2 L+1) \varphi(\lambda+2, L) \chi(\lambda+1, L-2)]^{\frac{1}{2}}}$ \\
\hline & $L-1$ & $\frac{\left\{(\lambda+3)^{2}(L-3)+(\lambda+3)(L-2)(L+1)+3(L-1)(L+1)\right\}[(\lambda+3)(\lambda-L+3)(L+2)]^{\frac{1}{2}}}{[(\lambda+1)(\lambda+6)(L-1) L(2 L+1) W(\lambda+1, L-1) \varphi(\lambda+2, L)]^{\frac{1}{2}}}$ \\
\hline$\stackrel{n}{n}$ & $L$ & $\frac{\left\{9(\lambda+3)^{2}-2(\lambda+3)\left(L^{2}+L-3\right)+9\right\}[(\lambda+3)(\lambda-L+3)(\lambda+L+4)(L-1)(L+2)]^{\frac{1}{2}}}{[3(\lambda+1)(\lambda+4)(\lambda+6) L(L+1)(2 L-1)(2 L+3) \varphi(\lambda+2, L) \gamma(\lambda+1, L)]^{\frac{1}{2}}}$ \\
\hline & $L+1$ & $-\frac{\left\{(\lambda+3)^{2}(L+4)-(\lambda+3) L(L+3)-3 L(L+2)\right\}[(\lambda+3)(\lambda+L+4)(L-1)]^{\frac{1}{2}}}{[(\lambda+1)(\lambda+6)(L+1)(L+2)(2 L+1) \varphi(\lambda+2, L) W(\lambda+1, L+1)]^{\frac{1}{2}}}$ \\
\hline & $L+2$ & $\frac{\left\{(\lambda+3)^{2}-(\lambda+1)(L+1)+2\right\}[2(\lambda+3)(\lambda+L+4)(\lambda+L+6)(L-1) L(L+3)]^{\frac{1}{1}}}{[(\lambda+1)(\lambda+4)(\lambda+6)(L+2)(2 L+1)(2 L+3) \varphi(\lambda+2, L) \chi(\lambda+1, L+2)]^{\frac{1}{2}}}$ \\
\hline & & $\lambda-L=$ odd \\
\hline & $L_{1}$ & $\left\langle(\lambda 3) \kappa=3 L_{1} ;(20) l \|(\lambda+1,2) \kappa=2 L\right\rangle$ \\
\hline$\stackrel{0}{11}$ & $L$ & {$\left[\frac{(\lambda+2)(L-2)(L+3) \varphi(\lambda+2, L)}{2(\lambda+1)(\lambda+4)(\lambda+6) \chi(\lambda+1, L)}\right]^{\frac{1}{2}}$} \\
\hline \multirow{5}{*}{$\stackrel{-11}{N}$} & $L-2$ & $-\left[\frac{3(\lambda+2)(\lambda-L+3)(\lambda+L+2)(L-4)(L-3)(L-2)(L+2) \varphi(\lambda+2, L)}{2(\lambda+1)(\lambda+4)(\lambda+6)(L-1) L(2 L-1)(2 L+1) \chi(\lambda+1, L-2)}\right]^{\frac{1}{2}}$ \\
\hline & $L-1$ & $-\frac{\{\lambda(L+5)+12\}[3(\lambda+L+2)(L-3)(L-2) \varphi(\lambda+2, L)]^{t}}{[4(\lambda+1)(\lambda+4)(\lambda+6)(L-1) L(L+1)(2 L+1) W(\lambda+1, L-1)]^{\frac{1}{t}}}$ \\
\hline & $L$ & $-\frac{\left\{15(\lambda+2)+2\left(L^{2}+L+3\right)\right\}[(\lambda+2)(L-2)(L+3) \varphi(\lambda+2, L)]^{\frac{1}{2}}}{[4(\lambda+1)(\lambda+4)(\lambda+6) L(L+1)(2 L-1)(2 L+3) \chi(\lambda+4, L)]^{\frac{1}{2}}}$ \\
\hline & $L+1$ & $\frac{\{(\lambda+2)(L-4)-2(L+2)\}[3(\lambda-L+1)(L+3)(L+4) \varphi(\lambda+2, L)]^{\frac{1}{2}}}{[4(\lambda+1)(\lambda+4)(\lambda+6) L(L+1)(L+2)(2 L+1) W(\lambda+1, L+1)]^{\frac{1}{t}}}$ \\
\hline & $L+2$ & {$\left[\frac{3(\lambda+2)(\lambda-L+1)(\lambda+L+4)(L+5)(L+4)(L+3)(L-1) \varphi(\lambda+2, L)}{2(\lambda+1)(\lambda+4)(\lambda+6)(L+1)(L+2)(2 L+1)(2 L+3) \chi(\lambda+1, L+2)}\right]^{\frac{1}{2}}$} \\
\hline
\end{tabular}

$\varphi(\lambda, L)=2(\lambda+1)^{2}-L(L+1), \quad \chi(\lambda, L)=4(\lambda+1)^{2}-(L-1)(L+2)$,

$W(\lambda, L)=4 \lambda(\lambda+2)-3(L-1)(L+2)$. 
TABLE 9 (continued)

\begin{tabular}{|c|c|c|}
\hline \multicolumn{3}{|r|}{$\lambda-L=$ odd } \\
\hline & $L_{1}$ & $\left\langle(\lambda 3)_{\kappa}=1 L_{1} ;(20) l||(\lambda-1,3)_{\kappa}=1 L\right\rangle$ \\
\hline i & $L$ & $-\left[\frac{2(\lambda-L+3)(\lambda+L+4) W(\lambda, L)}{45(\lambda+1)(\lambda+5) \chi(\lambda+1, L)}\right]^{\frac{1}{2}}$ \\
\hline \multirow{5}{*}{$\underline{-1}$} & $L-2$ & $\frac{Z(\lambda, L-1)[6(\lambda-L+3)(\lambda-L+5)(L-2)(L+1)]^{\ddagger}}{[5(\lambda+1)(\lambda+5)(2 L-1)(2 L+1) \chi(\lambda+1, L-2) W(\lambda, L)]^{!}}$ \\
\hline & $L-1$ & $\frac{\{4(\lambda-1)(\lambda+3)(2 L+1)-9(L+1)(L+3)(L-2)\}[(\lambda+4)(\lambda-L+3)]^{\frac{1}{3}}}{[15(\lambda+1)(\lambda+5) L(2 L+1) W(\lambda+1, L-1) W(\lambda, L)]^{\frac{1}{2}}}$ \\
\hline & $L$ & $\frac{\left\{4(\lambda-1)(\lambda+3)\left(5 L^{2}+5 L+3\right)-3(L-2)\left(2 L^{3}+8 L^{2}+15 L+27\right)\right\}[(\lambda-L+3)(\lambda+L+4)]^{\frac{f}{2}}}{[45(\lambda+1)(\lambda+5) L(L+1)(2 L-1)(2 L+3) W(\lambda, L) \chi(\lambda+1, L)]^{\frac{1}{3}}}$ \\
\hline & $L+1$ & $\frac{\{4(\lambda-1)(\lambda+3)(2 L+1)-9 L(L-2)(L+3)\}[(\lambda+4)(\lambda+L+4)]^{\ddagger}}{[15(\lambda+1)(\lambda+5)(L+1)(2 L+1) W(\lambda, L) W(\lambda+1, L+1)]^{\frac{1}{t}}}$ \\
\hline & $L+2$ & $\frac{Z(\lambda, L+1)[6(\lambda+L+4)(\lambda+L+6) L(L+3)]^{\frac{1}{2}}}{[5(\lambda+1)(\lambda+5)(2 L+1)(2 L+3) \chi(\lambda+1, L+2) W(\lambda, L)]^{\frac{1}{t}}}$ \\
\hline
\end{tabular}

$$
\lambda-L=\text { odd }
$$

\begin{tabular}{|c|c|c|}
\hline & $L_{1}$ & $\left\langle(\lambda 3) \kappa=3 L_{1} ;(20) l \|(\lambda-1,3) \kappa=1 L\right\rangle$ \\
\hline il & $L$ & $-\left[\frac{8(\lambda+2)(\lambda+3)(L-2)(L-1)(L+2)(L+3)}{15(\lambda+1)(\lambda+5) W(\lambda, L) \chi(\lambda+1, L)}\right]^{\frac{1}{2}}$ \\
\hline \multirow{5}{*}{$\stackrel{-1}{11}$} & $L-2$ & $-\frac{(\lambda-L-1)[8(\lambda+2)(\lambda+3)(\lambda-L+3)(\lambda+L+2)(L-4)(L-3)(L-2)]^{t}}{[5(\lambda+1)(\lambda+5) L(2 L+1)(2 L-1) W(\lambda, L) \chi(\lambda+1, L-2)]^{t}}$ \\
\hline & $L-1$ & $-\frac{2\{2(\lambda+2)(\lambda-L-1)+3(L-1)\}[(\lambda+3)(\lambda+L+2)(L-3)(L-2)(L+2)]^{\sharp}}{[5(\lambda+1)(\lambda+5) L(L+1)(2 L+1) W(\lambda, L) W(\lambda+1, L-1)]^{t}}$ \\
\hline & $L$ & $-\frac{2\{6(\lambda-1)(\lambda+2)-2 L(L+1)-9\}[(\lambda+2)(\lambda+3)(L-2)(L-1)(L+2)(L+3)]^{\mid}}{[15(\lambda+1)(\lambda+5) L(L+1)(2 L-1)(2 L+3) W(\lambda, L) \chi(\lambda+1, L)]^{\mid}}$ \\
\hline & $L+1$ & $-\frac{2\{2(\lambda+2)(\lambda+L)-3(L+2)\}[(\lambda+3)(\lambda-L+1)(L-1)(L+3)(L+4)]}{[5(\lambda+1)(\lambda+5) L(L+1)(2 L+1) W(\lambda, L) W(\lambda+1, L+1)]^{t}}$ \\
\hline & $L+2$ & $-\frac{(\lambda+L)[8(\lambda+2)(\lambda+3)(\lambda-L+1)(\lambda+L+4)(L+5)(L+4)(L+3)]^{\frac{1}{3}}}{[5(\lambda+1)(\lambda+5)(L+1)(2 L+1)(2 L+3) W(\lambda, L) \chi(\lambda+1, L+2)]^{t}}$ \\
\hline
\end{tabular}

$\chi(\lambda, L)=4(\lambda+1)^{2}-(L-1)(L+2)$,

$W(\lambda, L)=4 \lambda(\lambda+2)-3(L-1)(L+2)$,

$Z(\lambda, L)=2 \lambda(\lambda+2)-(L+3)(L-2)$. 
TABLE 9 (continued)

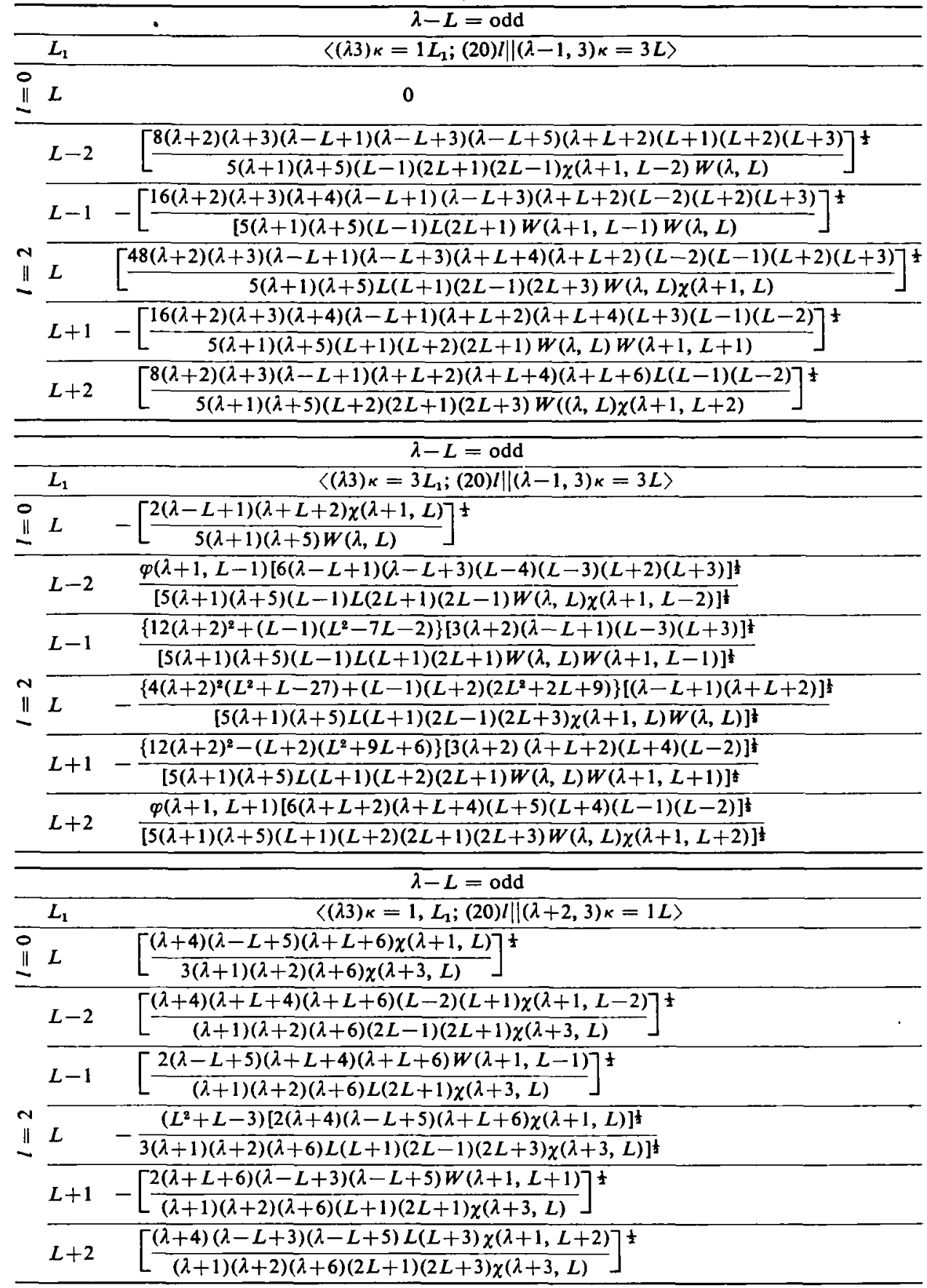

$\left\langle(\lambda 3) \kappa=3 L_{1} ;(20) l||(\lambda+2,3) \kappa=1 L\right\rangle=0 \quad$ All $L_{1}, \lambda-L=$ odd

$W(\lambda, L)=4 \lambda(\lambda+2)-3(L-1)(L+2)$,

$\chi(\lambda, L)=4(\lambda+1)^{2}-(L-1)(L+2)$,

$\varphi(\lambda, L)=2(\lambda+1)^{2}-L(L+1)$. 
TABLE 9 (continued)

\begin{tabular}{|c|c|}
\hline & $\lambda-L=$ odd \\
\hline & $\left\langle(\lambda 3) \kappa=1 L_{1} ;(20) l \|(\lambda+2,3) \kappa=3 L\right\rangle$ \\
\hline$\stackrel{0}{11}$ & {$\left[\frac{16(\lambda+3)^{2}(L-2)(L-1)(L+2)(L+3)}{(\lambda+1)(\lambda+2)(\lambda+5)(\lambda+6) \chi(\lambda+1, L) \chi(\lambda+3, L)}\right]^{\frac{1}{2}}$} \\
\hline \multirow{5}{*}{$\sqrt{n}$} & $L-2-\frac{\{(\lambda+2)(2 L-5)+3(L-3)\}[12(\lambda-L+5)(\lambda+L+4)(L+1)(L+2)(L+3)]^{t}}{[(\lambda+1)(\lambda+2)(\lambda+5)(\lambda+6)(L-1)(2 L-1)(2 L+1) \chi(\lambda+3, L) \chi(\lambda+1, L-2)]^{t}}$ \\
\hline & $L-1 \quad \frac{2\{(\lambda+4)(L-4)+2(L-1)\}[6(\lambda+4)(\lambda+L+4)(L-2)(L+2)(L+3)]^{t}}{[(\lambda+1)(\lambda+2)(\lambda+5)(\lambda+6) L(L-1)(2 L+1) \chi(\lambda+3, L) W(\lambda+1, L-1)]^{t}}$ \\
\hline & $L \quad \frac{2\left\{12 \lambda^{2}+102 \lambda+216-(2 \lambda+9) L(L+1)\right\}[2(L-2)(L-1)(L+2)(L+3)]^{\mid}}{[(\lambda+1)(\lambda+2)(\lambda+5)(\lambda+6) L(L+1)(2 L-1)(2 L+3) \chi(\lambda+1, L) \chi(\lambda+3, L)]^{\frac{1}{2}}}$ \\
\hline & $L+1-\frac{2\{(\lambda+4)(L+5)+2(L+2)\}[6(\lambda+4)(\lambda-L+3)(L-2)(L-1)(L+3)]^{\ddagger}}{[(\lambda+1)(\lambda+2)(\lambda+5)(\lambda+6)(L+1)(L+2)(2 L+1) \chi(\lambda+3, L) W(\lambda+1, L+1)]^{\frac{1}{4}}}$ \\
\hline & $L+2 \quad \frac{\{(\lambda+2)(2 L+7)+3(L+4)\}[12(\lambda-L+3)(\lambda+L+6)(L-2)(L-1) L]^{\frac{1}{t}}}{[(\lambda+1)(\lambda+2)(\lambda+5)(\lambda+6)(L+2)(2 L+1)(2 L+3) \chi(\lambda+3, L) \chi(\lambda+1, L+2)]^{\frac{1}{2}}}$ \\
\hline & $\lambda-L=$ odd \\
\hline & $\left\langle(\lambda 3) \kappa=3 L_{1} ;(20) l \|(\lambda+2,3) \kappa=3 L\right\rangle$ \\
\hline iI & {$\left[\frac{(\lambda+3)(\lambda-L+3)(\lambda+L+4) \chi(\lambda+3, L)}{3(\lambda+1)(\lambda+5)(\lambda+6) \chi(\lambda+1, L)}\right]^{ \pm}$} \\
\hline \multirow{5}{*}{ II } & $L-2 \quad\left[\frac{(\lambda+3)(\lambda+L+2)(\lambda+L+4)(L-4)(L-3)(L+2)(L+3) \chi(\lambda+3, L)}{(\lambda+1)(\lambda+5)(\lambda+6)(L-1) L(2 L+1)(2 L-1) \chi(\lambda+1, L-2)}\right]^{\frac{1}{2}}$ \\
\hline & $L-1 \quad\left[\frac{18(\lambda+3)(\lambda-L+3)(\lambda+L+2)(\lambda+L+4)(L-3)(L+3) \chi(\lambda+3, L)}{(\lambda+1)(\lambda+2)(\lambda+5)(\lambda+6)(L-1) L(L+1)(2 L+1) W(\lambda+1, L-1)}\right]^{\frac{1}{2}}$ \\
\hline & $L \quad-\frac{\left(L^{2}+L-27\right)[2(\lambda+3)(\lambda-L+3)(\lambda+L+4) \chi(\lambda+3, L)]^{\frac{1}{3}}}{[3(\lambda+1)(\lambda+5)(\lambda+6) L(L+1)(2 L-1)(2 L+3) \chi(\lambda+1, L)]^{\frac{1}{3}}}$ \\
\hline & $L+1-\left[\frac{18(\lambda+3)(\lambda-L+1)(\lambda-L+3)(\lambda+L+4)(L-2)(L+4) \chi(\lambda+3, L)}{(\lambda+1)(\lambda+2)(\lambda+5)(\lambda+6) L(L+1)(L+2)(2 L+1) W(\lambda+1, L+1)}\right]^{\frac{1}{2}}$ \\
\hline & $L+2 \quad\left[\frac{(\lambda+3)(\lambda-L+1)(\lambda-L+3)(L+5)(L+4)(L-1)(L-2) \chi(\lambda+3, L)}{(\lambda+1)(\lambda+5)(\lambda+6)(L+1)(L+2)(2 L+1)(2 L+3) \chi(\lambda+1, L+2)}\right]^{\frac{1}{2}}$ \\
\hline
\end{tabular}

$\chi(\lambda, L)=4(\lambda+1)^{2}-(L-1)(L+2), \quad W(\lambda, L)=4 \lambda(\lambda+2)-3(L-1)(L+2)$. 
TABLe 10

$\left\langle(\lambda 0) L_{1} ;(11) l \|\left(\lambda^{\prime} \mu^{\prime}\right) \kappa L\right\rangle$

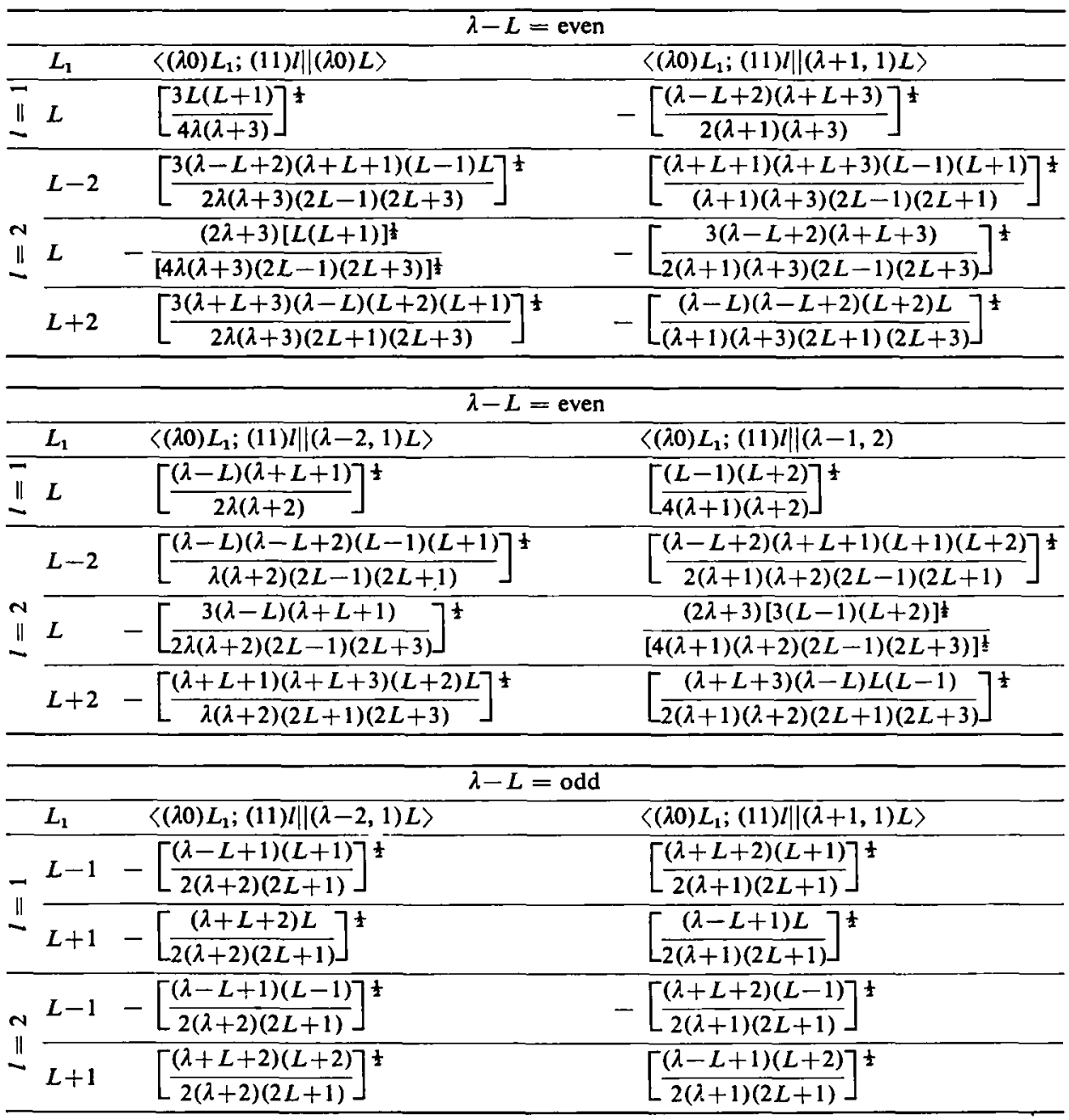

\begin{tabular}{|c|c|c|c|}
\hline \multicolumn{4}{|c|}{$\lambda-L=$ odd } \\
\hline & $L_{1}$ & $\left\langle(\lambda 0) L_{1} ;(11) l||(\lambda-1,2) \kappa=0 L\right\rangle$ & $\left\langle(\lambda .0) L_{1} ;(11) l \|(\lambda-1,2) \kappa=2 L\right\rangle$ \\
\hline \multirow{4}{*}{$\bar{I}$} & & $(2 \lambda-L+1)[(\lambda+L+2) L]^{\frac{1}{2}}$ & {$[(\lambda-L+1)(L-1)(L+1)(L+2)]^{\frac{1}{2}}$} \\
\hline & & {$[2(\lambda+1)(2 L+1) \varphi(\lambda, L)]^{\frac{1}{2}}$} & {$\left[\frac{2(\lambda+2)(2 L+1) \varphi(\lambda, L)}{2}\right]$} \\
\hline & & $(2 \lambda+L+2)[(\lambda-L+1)(L+1)]^{\frac{1}{2}}$ & {$[(\lambda+L+2)(L+2) L(L-1)]^{\frac{1}{2}}$} \\
\hline & $L-$ & {$[2(\lambda+1)(2 L+1) \varphi(\lambda, L)]^{\frac{1}{2}}$} & $-[\overline{2(\lambda+2)(2 L+1) \varphi(\lambda, L)}]$ \\
\hline \multirow{4}{*}{$\stackrel{N}{\|}$} & & {$[(\lambda+L+2)(L-1) L(L+1)]^{\frac{1}{2}}$} & $(2 \lambda+L+3)[(\lambda-L+1)(L+2)]^{\frac{1}{2}}$ \\
\hline & & $\lfloor\overline{2(\lambda+1)(2 L+1) q(\lambda, L)}\rfloor$ & {$[2(\lambda+2)(2 L+1) \varphi(\lambda, L)]^{\frac{1}{2}}$} \\
\hline & & {$[(\lambda-L+1)(L+2) L(L+1)]^{\frac{1}{2}}$} & $(2 \lambda-L+2)[(\lambda+L+2)(L-1)]^{\frac{1}{2}}$ \\
\hline & & {$[\overline{2(\lambda+1)(2 L+1) \varphi(\lambda, L)}]$} & {$[2(\lambda+2)(2 L+1) \varphi(\lambda, L)]^{\frac{1}{2}}$} \\
\hline
\end{tabular}
$\varphi(\lambda, L)=2(\lambda+1)^{2}-L(L+1)$. 


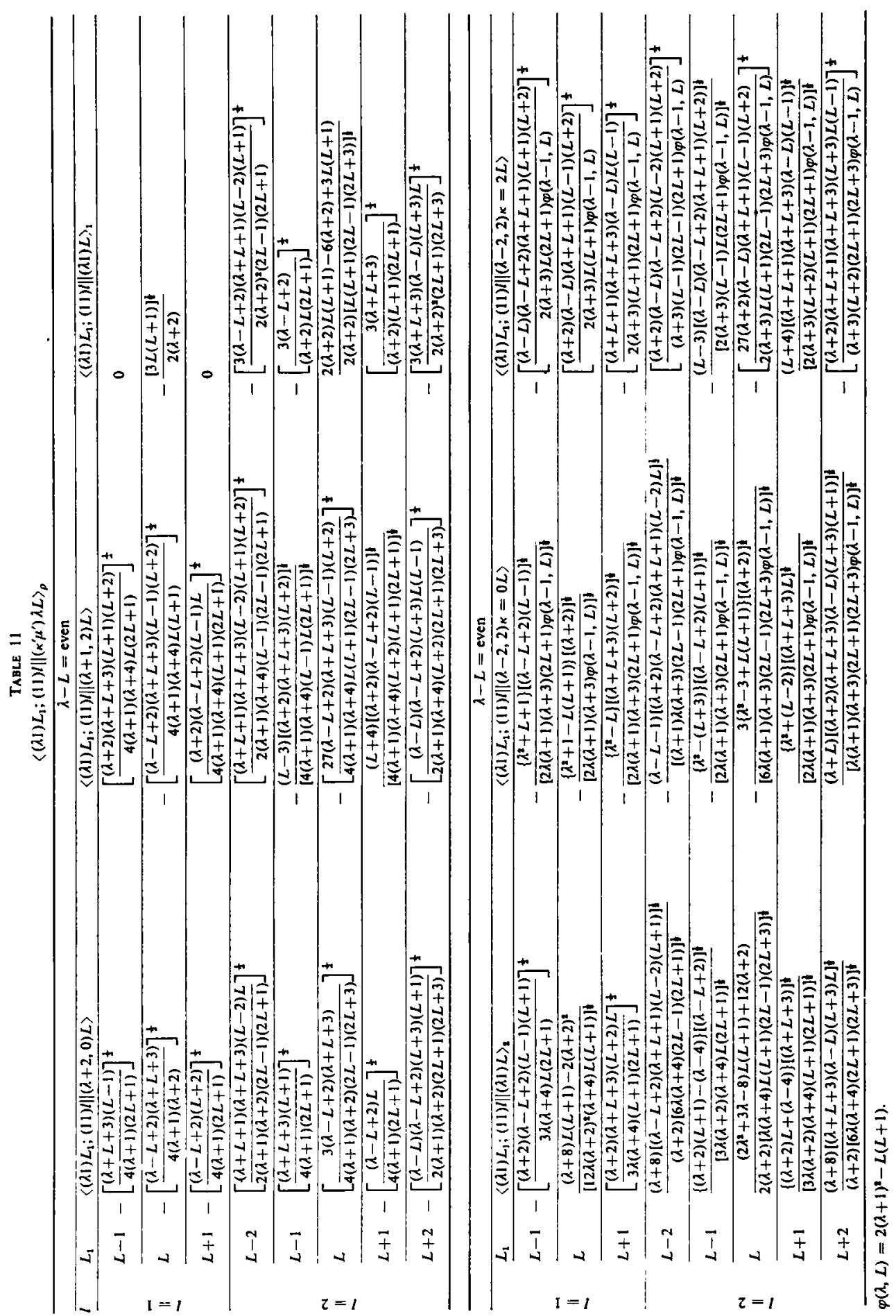




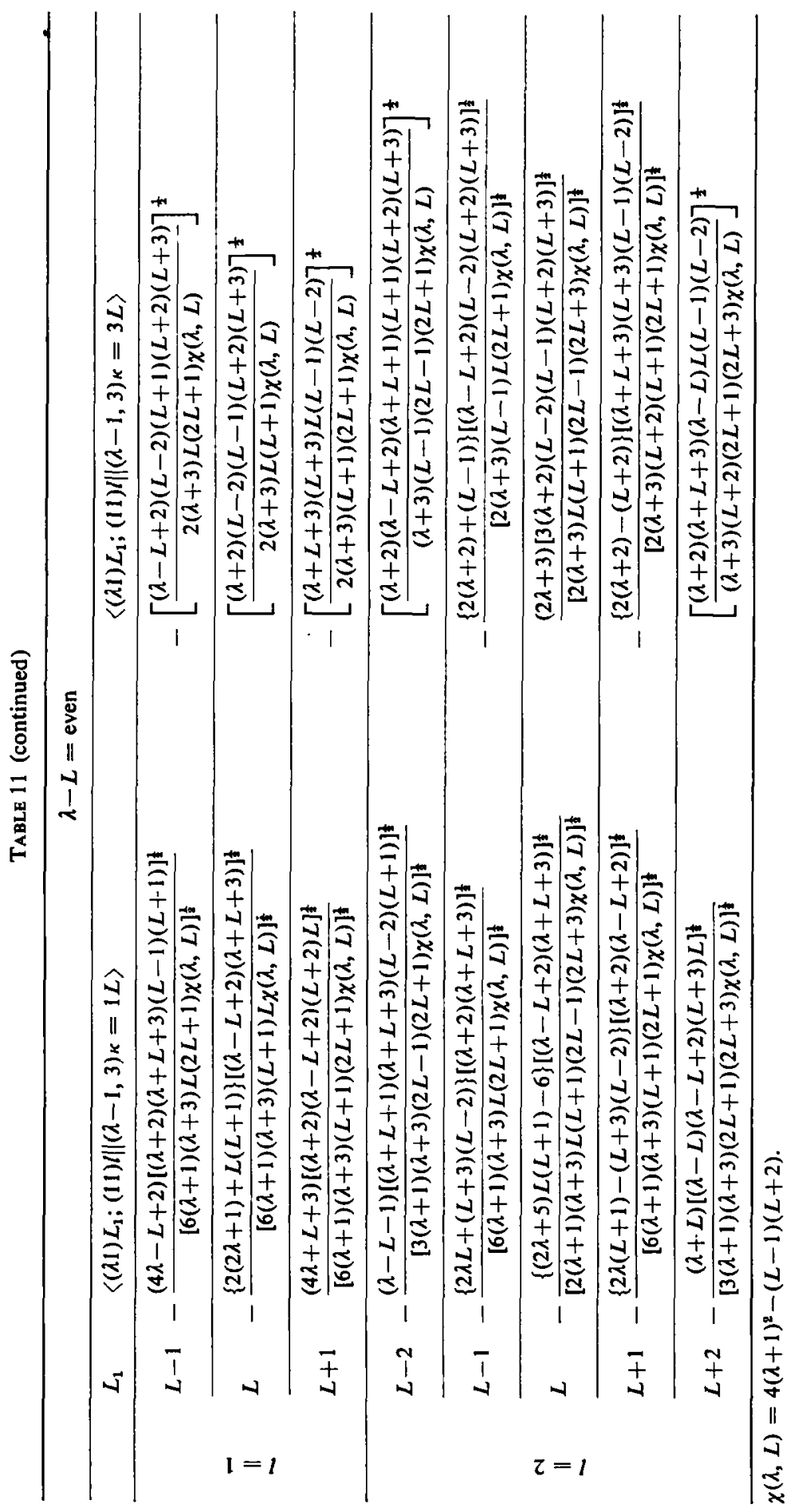




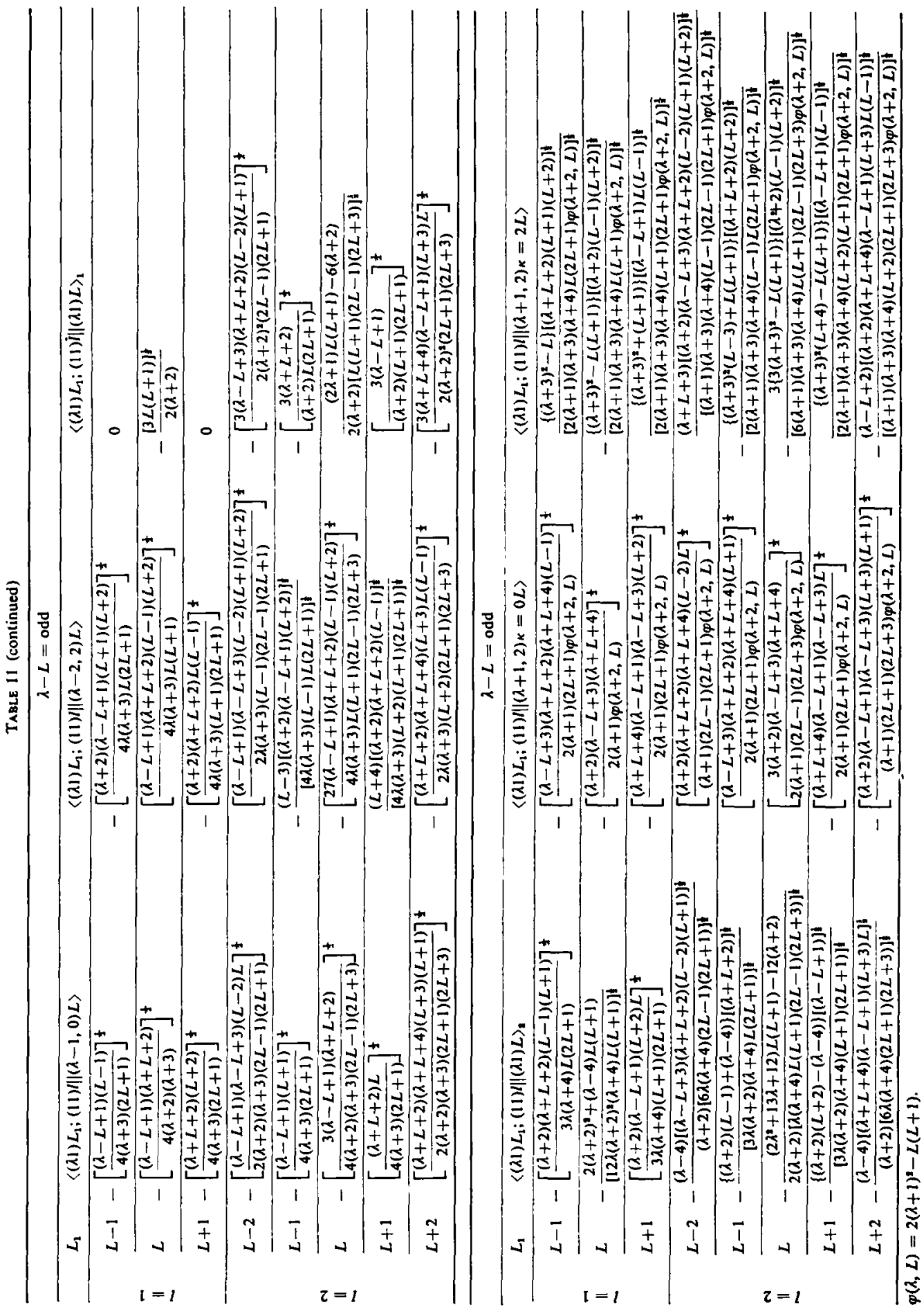




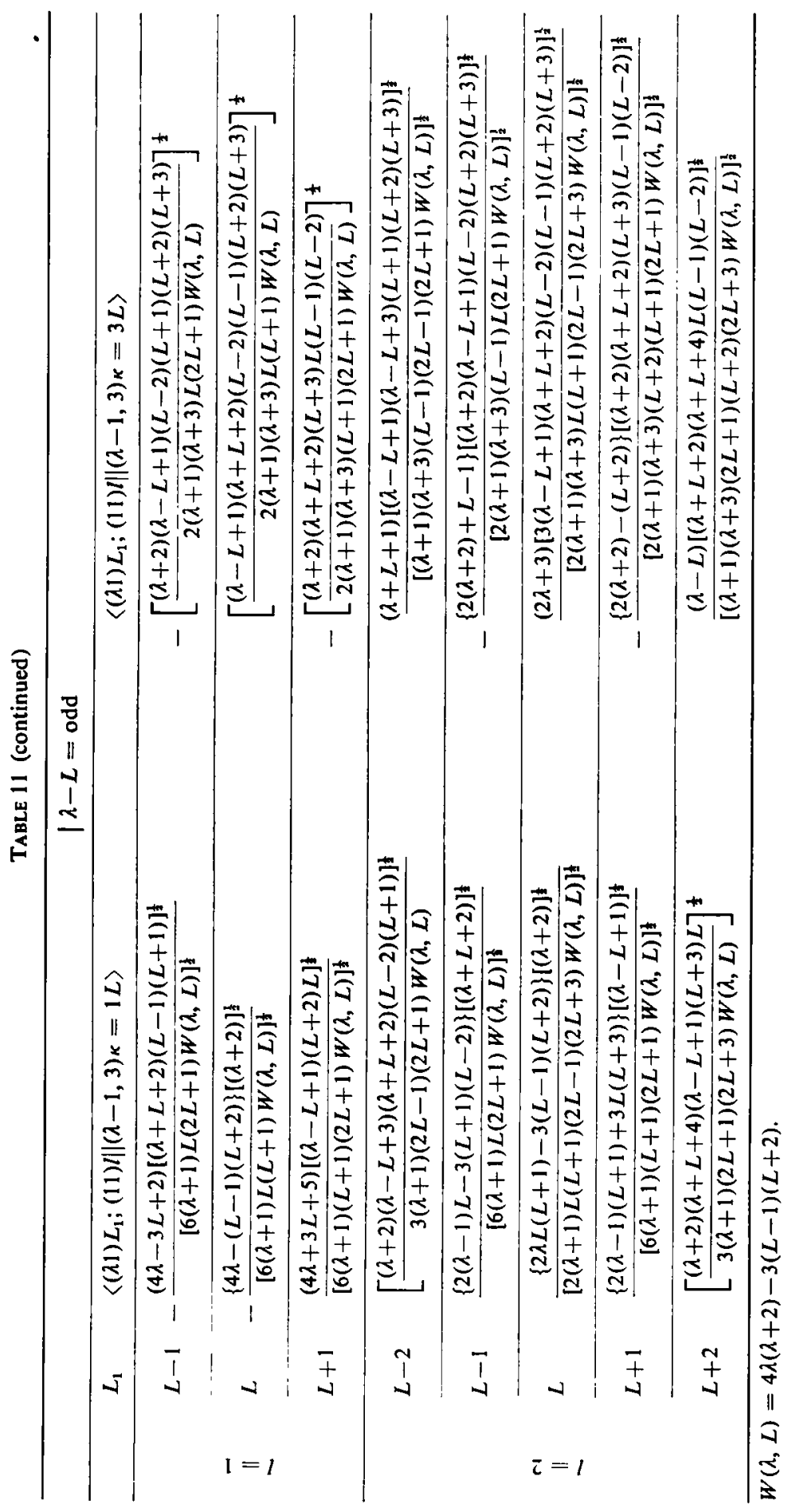




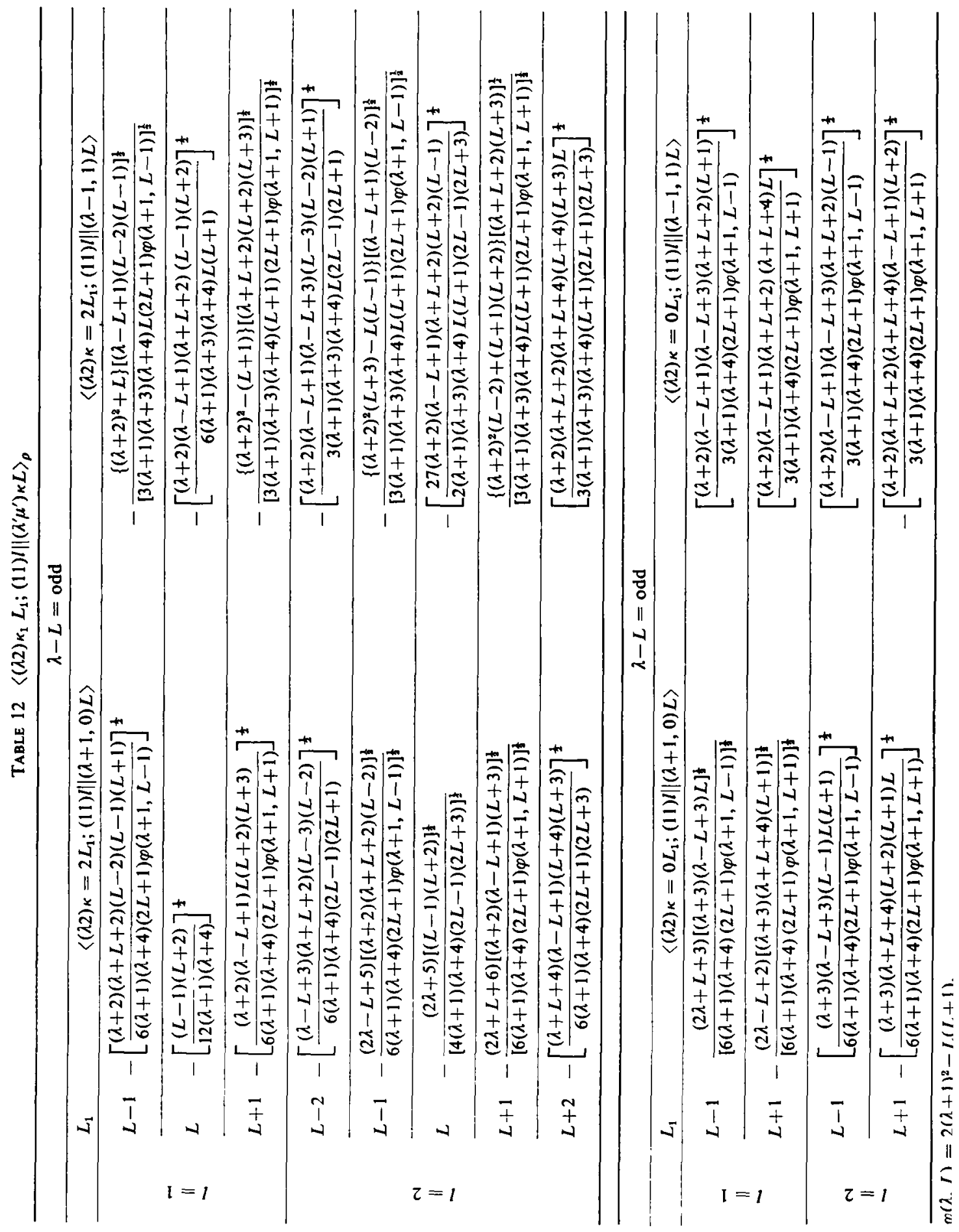




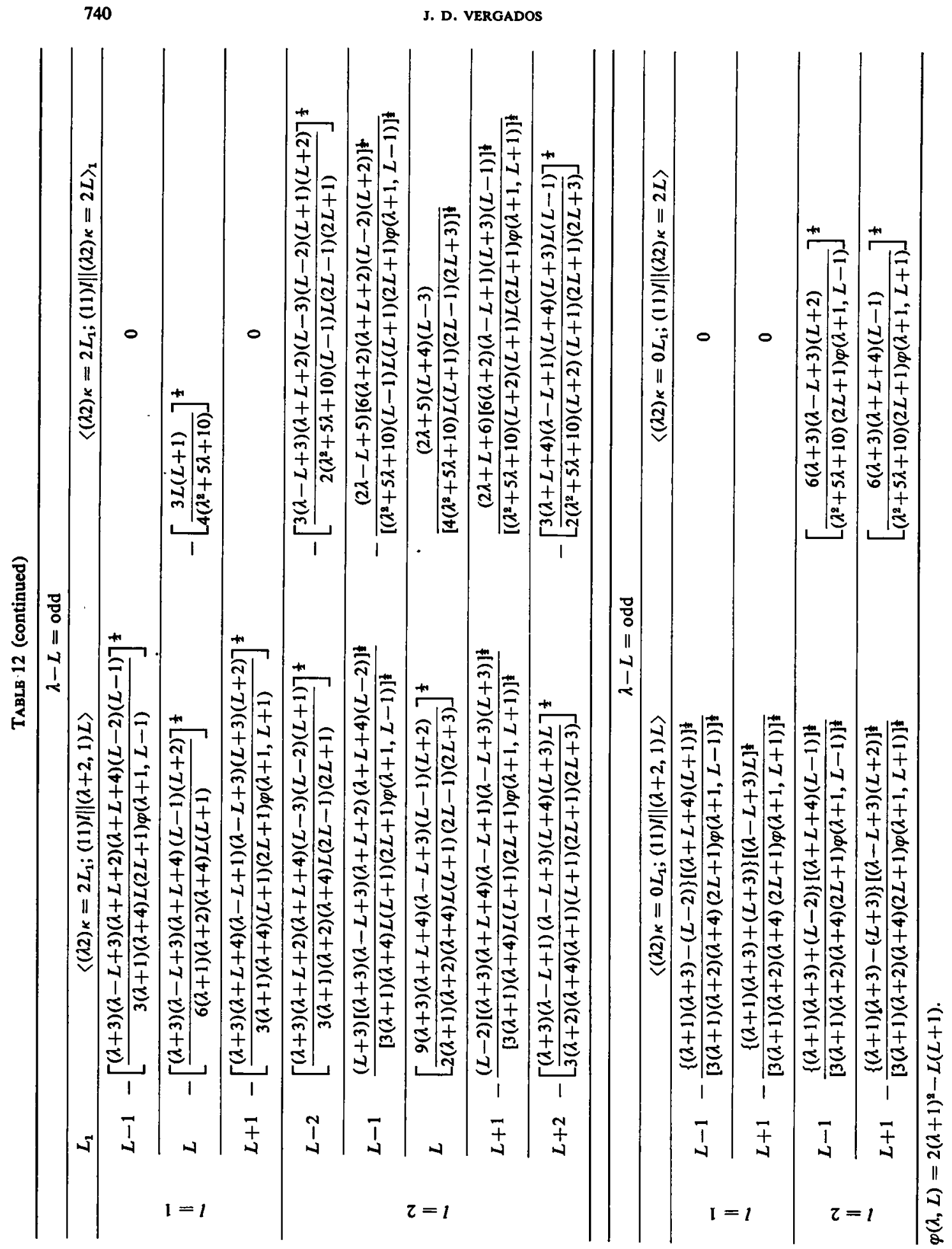


TABLE 12 (continued)

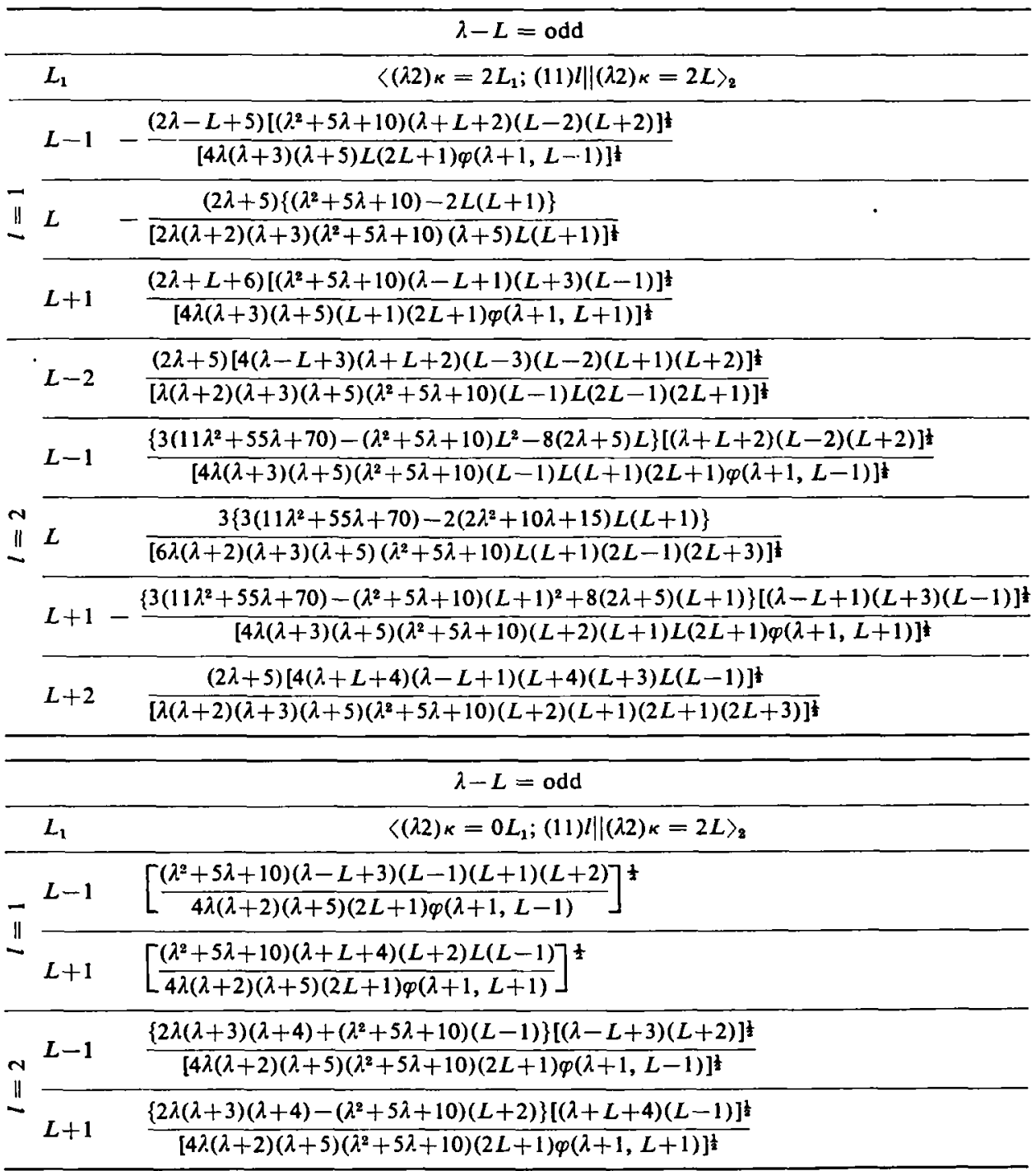

$\varphi(\lambda, L)=2(\lambda+1)^{2}-L(L+1)$. 


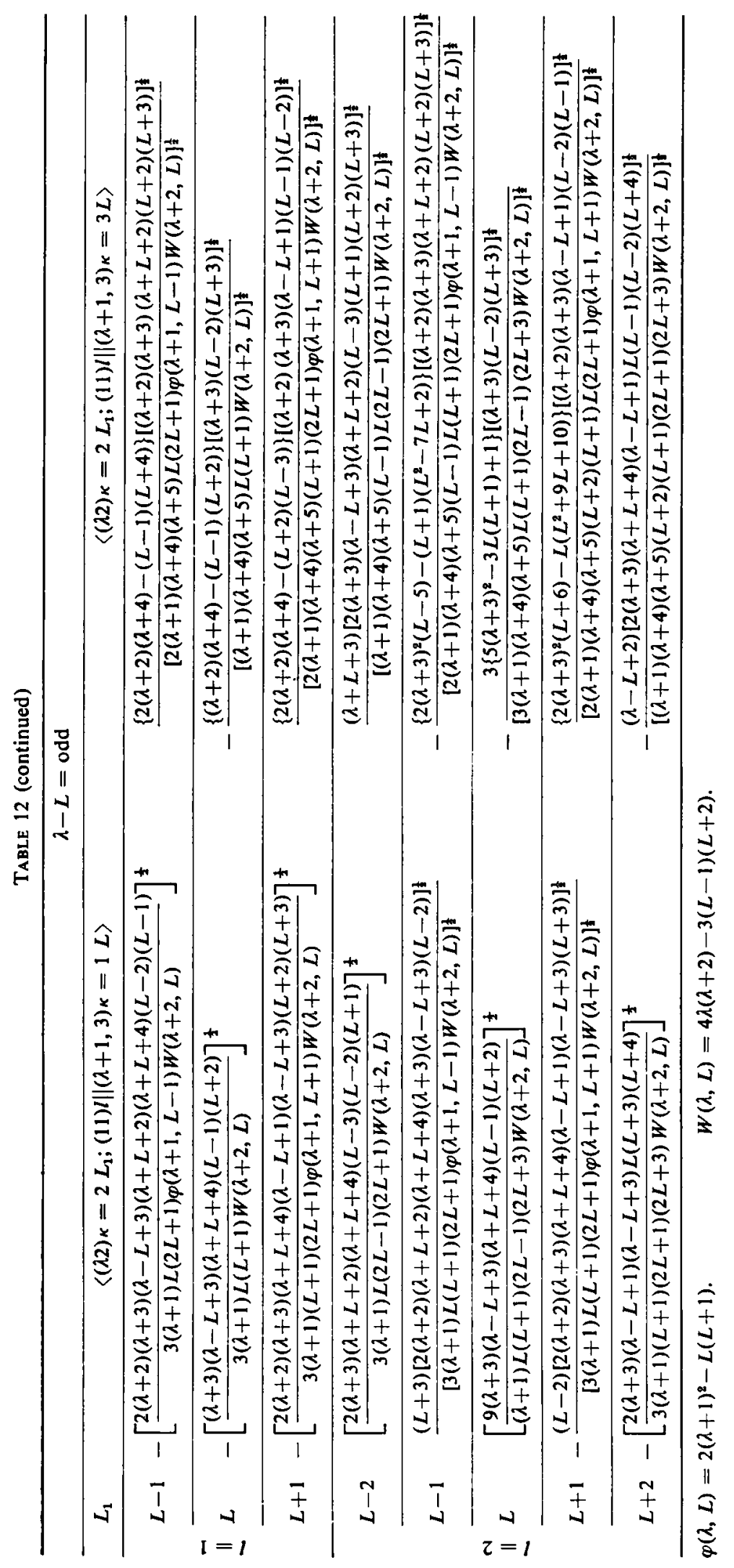



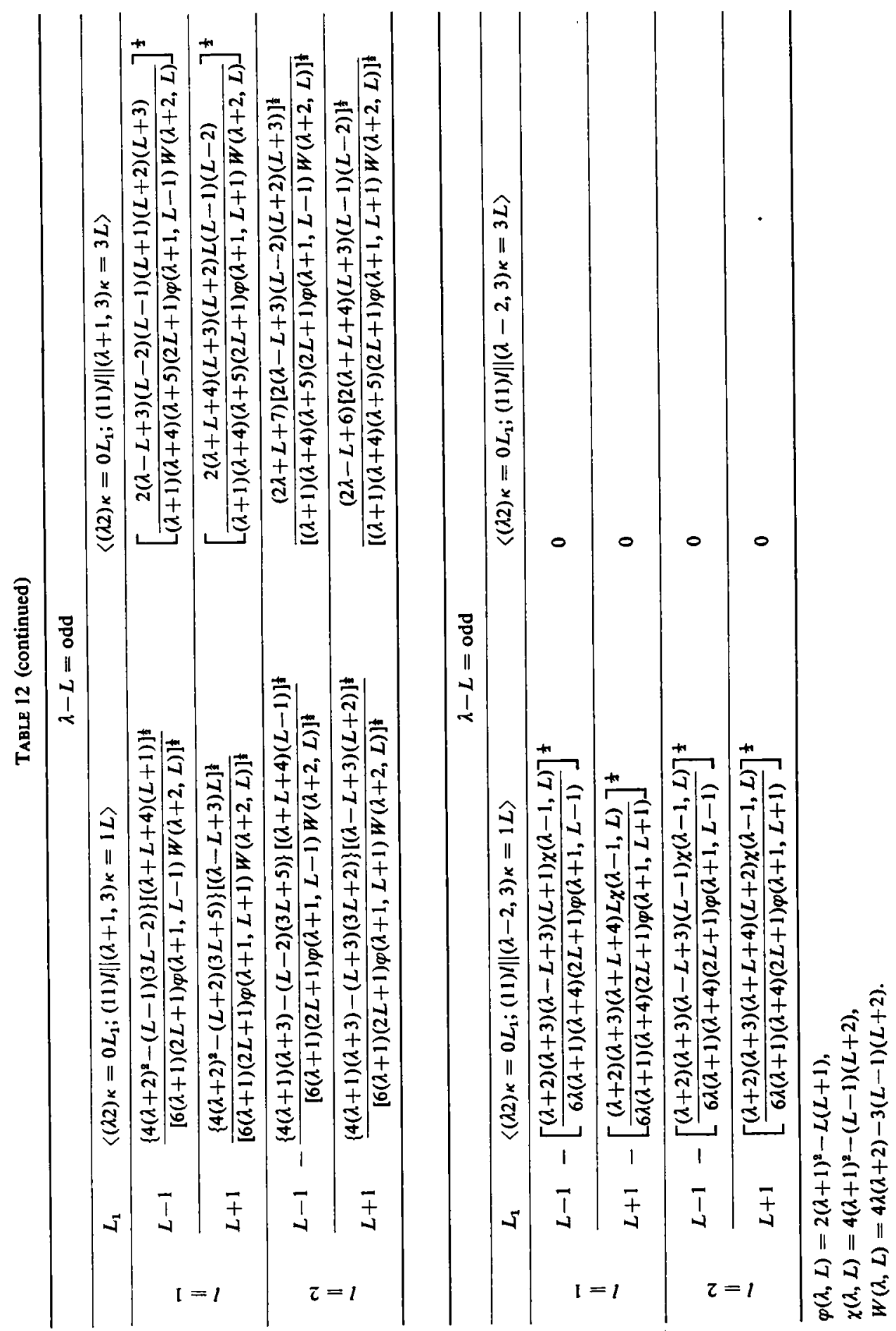


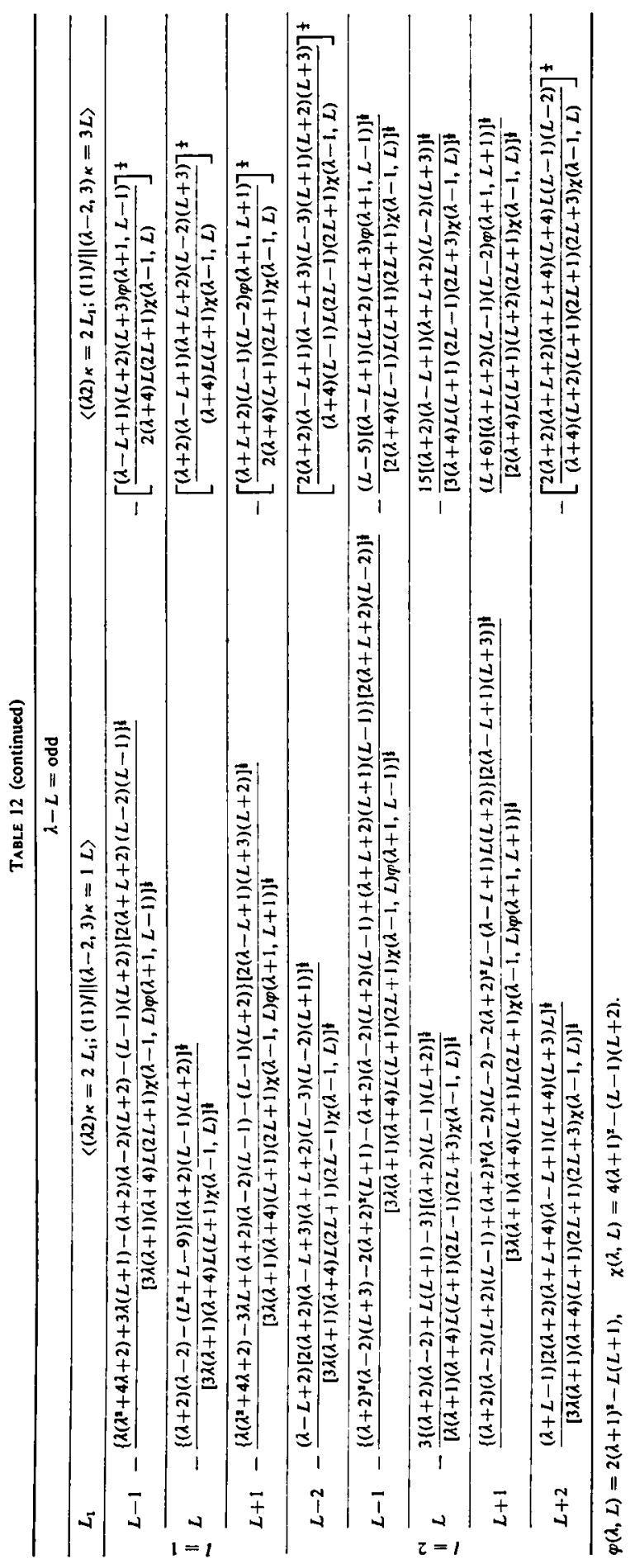




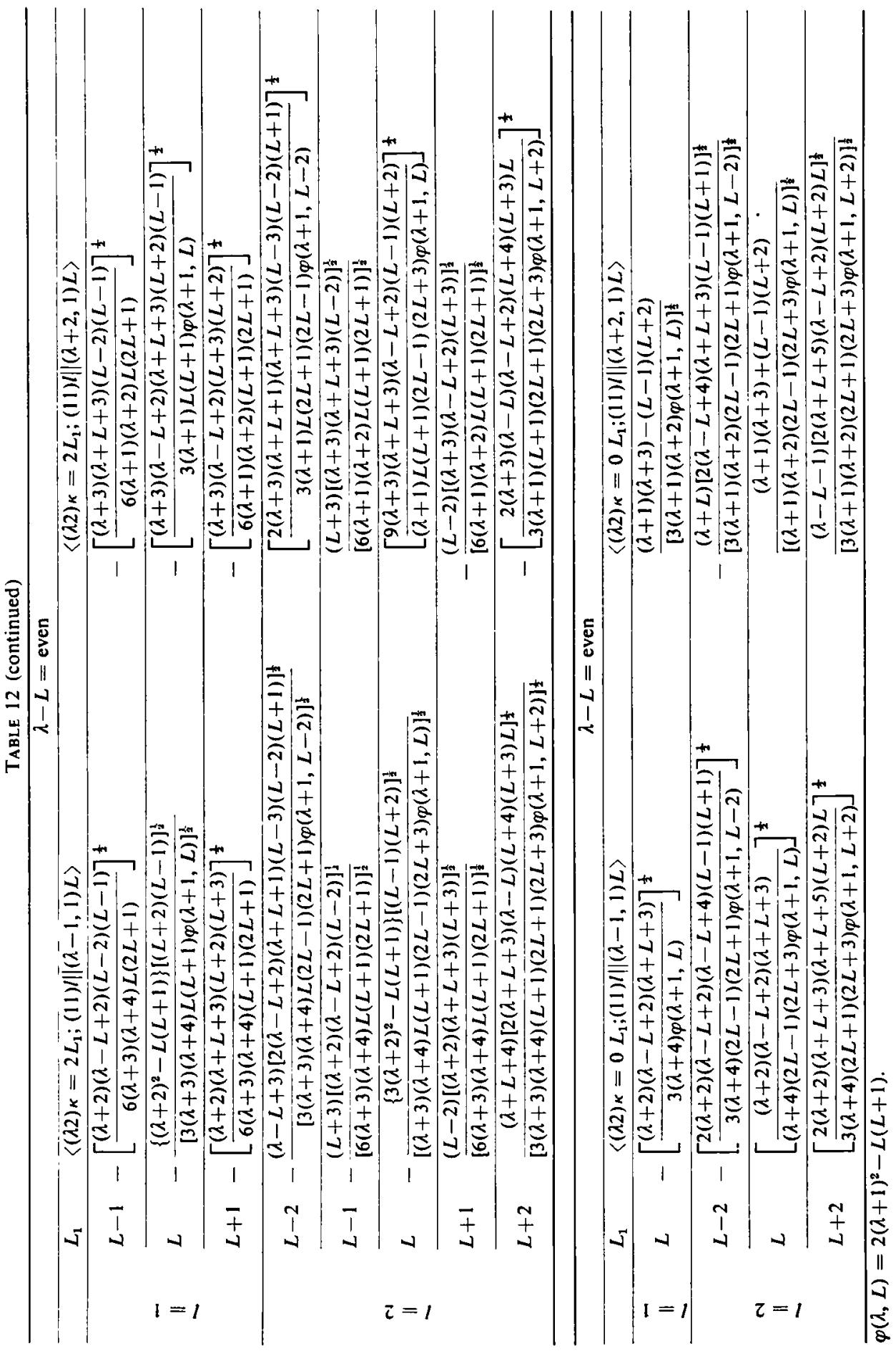




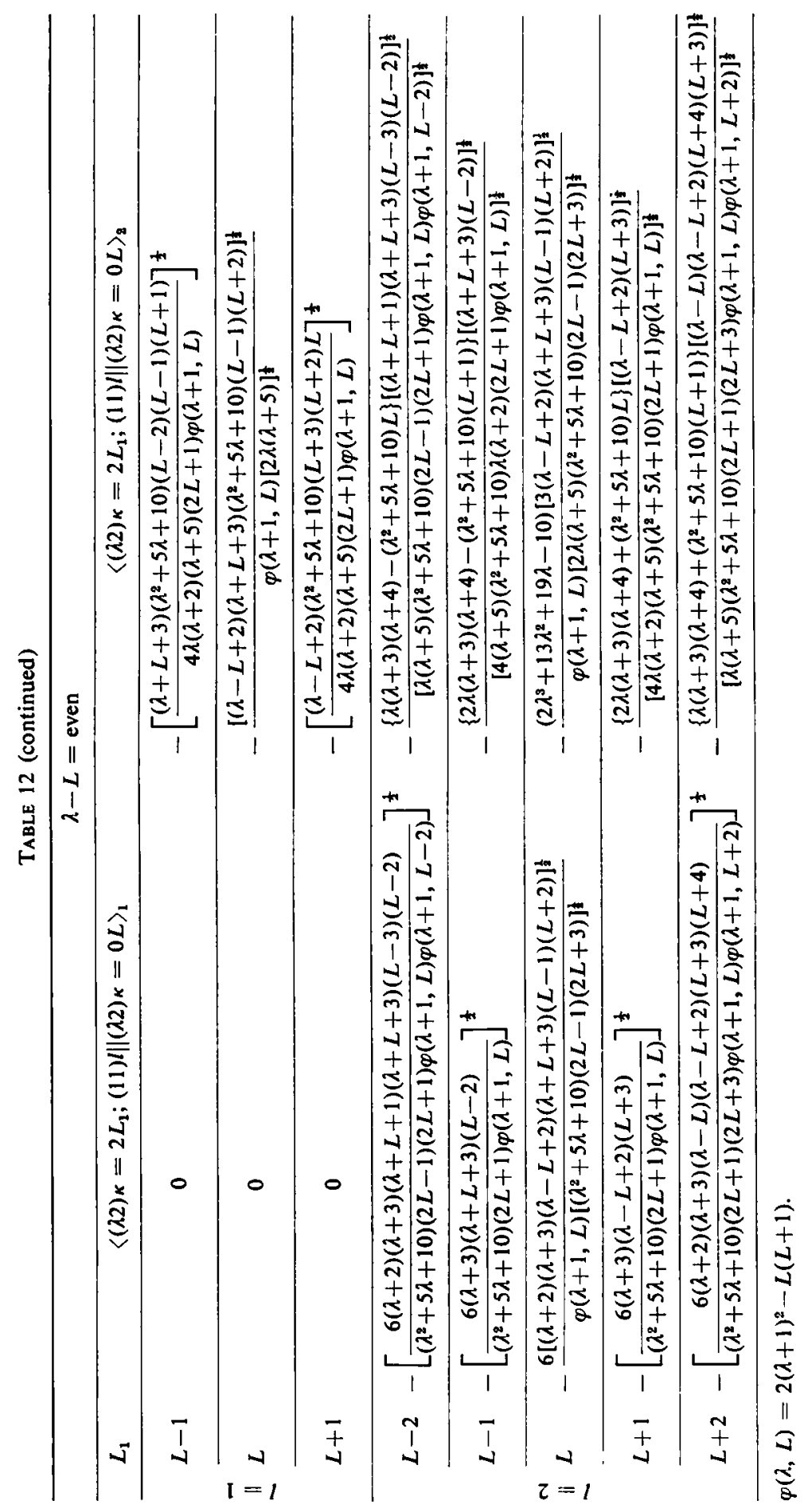


TABLE 12 (continued)

\begin{tabular}{|c|c|c|}
\hline \multicolumn{3}{|r|}{$\lambda-L=$ even } \\
\hline & $L_{1}$ & $\left\langle(\lambda 2) \kappa=2 L_{1} ;(11) l \|(\lambda 2) \kappa=2 L\right\rangle_{1}$ \\
\hline \multirow{3}{*}{$\overline{\|}$} & $L-1$ & 0 \\
\hline & $L$ & $-\frac{3\left\{2(\lambda+2)\left(4 \lambda-L^{2}-L+14\right)+A(\lambda, L)-6(L-1)(L+2)\right\}}{2 \varphi(\lambda+1, L)\left[3\left(\lambda^{2}+5 \lambda+10\right) L(L+1)\right]^{\ddagger}}$ \\
\hline & $L+1$ & 0 \\
\hline \multirow{5}{*}{$\stackrel{N}{I I}$} & $L-2$ & $-\frac{\{2(\lambda+2)(\lambda+3)-L(L-1)\}[3(\lambda-L+2)(\lambda+L+1)(L-3)(L-2)(L+1)(L+2)]^{\mid}}{\left[2\left(\lambda^{2}+5 \lambda+10\right)(L-1) L(2 L-1)(2 L+1) \varphi(\lambda+1, L) \varphi(\lambda+1, L-2)\right]^{\mid}}$ \\
\hline & $L-1$ & $-\frac{(2 \lambda+L+5)[6(\lambda+2)(\lambda-L+2)(L-2)(L+2)]^{\frac{1}{2}}}{\left[\left(\lambda^{2}+5 \lambda+10\right)(L-1) L(L+1)(2 L+1) \varphi(\lambda+1, L)\right]^{\frac{1}{2}}}$ \\
\hline & $L$ & $-\frac{\left\{2(\lambda+2) B(\lambda, L)-C(\lambda, L)-2(L-1)(L+2)\left(4 L^{2}+4 L-21\right)\right.}{\varphi(\lambda+1, L)\left[4\left(\lambda^{2}+5 \lambda+10\right) L(L+1)(2 L-1)(2 L+3)\right]^{\ddagger}}$ \\
\hline & $L+1$ & $\frac{(2 \lambda-L+4)[6(\lambda+2)(\lambda+L+3)(L+3)(L-1)]^{\ddagger}}{\left[\left(\lambda^{2}+5 \lambda+10\right)(L+2)(L+1) L(2 L+1) \varphi(\lambda+1, L)\right]^{\ddagger}}$ \\
\hline & $L+2$ & $-\frac{\{2(\lambda+2)(\lambda+3)-(L+1)(L+2)\}[3(\lambda-L)(\lambda+L+3)(L+4)(L+3) L(L-1)]^{\mid}}{\left[2\left(\lambda^{2}+5 \lambda+10\right)(L+2)(L+1)(2 L+1)(2 L+3) \varphi(\lambda+1, L) \varphi(\lambda+1, L+2)\right]^{ \pm}}$ \\
\hline \multicolumn{3}{|c|}{$\begin{aligned} \varphi(\lambda, L)= & 2(\lambda+1)^{2}-L(L+1) \\
A(\lambda, L)= & 2(\lambda+1)^{2}\left(L^{2}+L-4\right)+2(\lambda+1)\left(3 L^{2}+3 L-14\right)-\left(L^{4}+2 L^{3}-9 L^{2}-10 L+32\right), \\
B(\lambda, L)= & 2(\lambda+4)(L+4)(L-3)+7 L^{2}+7 L+6 \\
C(\lambda, L)= & 4(\lambda+1)^{3}(L+4)(L-3)+18(\lambda+1)^{2}(L+4)(L-3)-2(\lambda+1)\left(L^{4}+2 L^{3}-34 L^{2}-35 L+186\right) \\
& -\left(5 L^{4}+10 L^{3}-109 L^{2}-114 L+288\right) .\end{aligned}$} \\
\hline
\end{tabular}




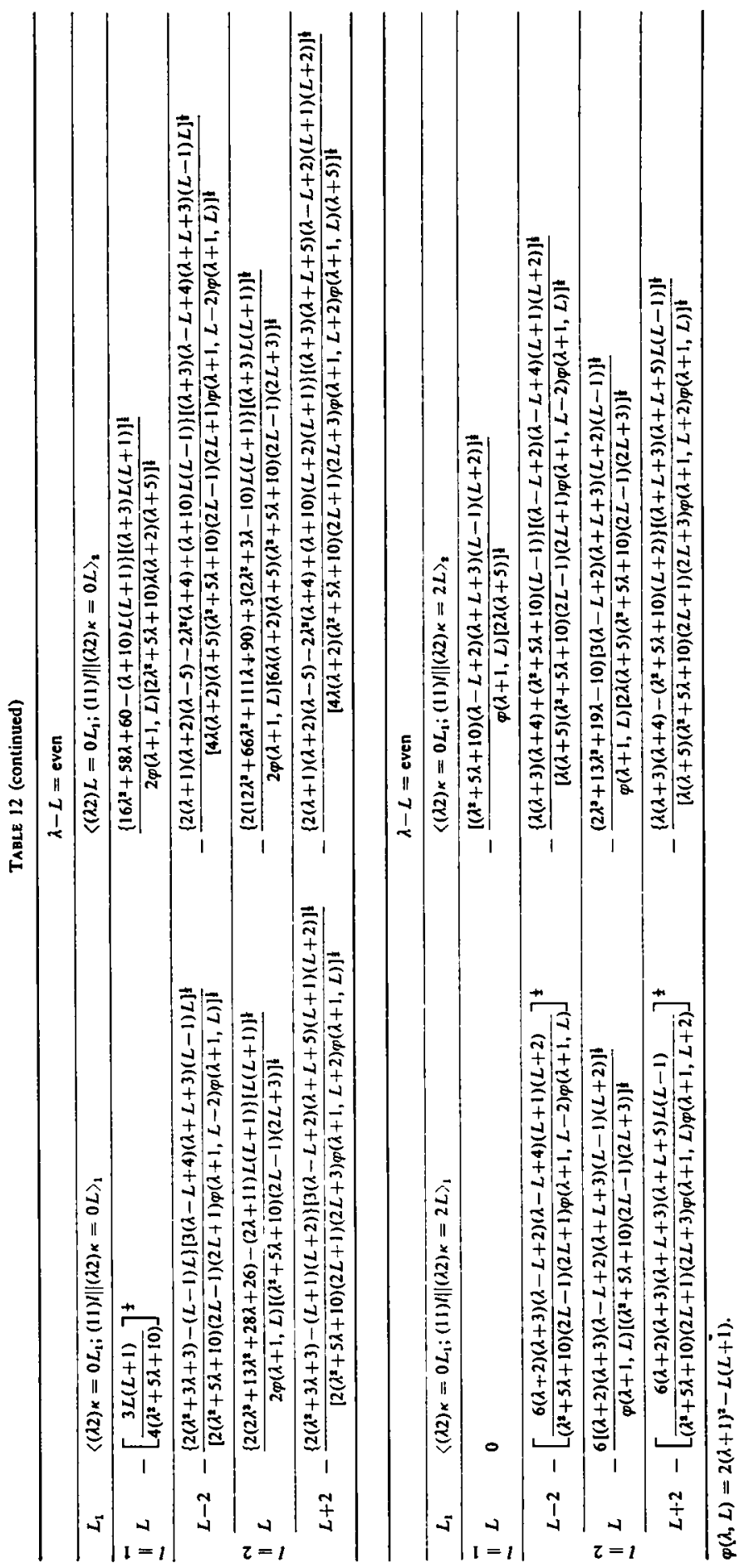


TABLE 12 (continued)

\begin{tabular}{|c|c|c|}
\hline \multicolumn{3}{|r|}{$\lambda-L=$ even } \\
\hline & $L_{1}$ & $\left\langle(\lambda 2)_{\kappa}=2 L_{1} ;(11) l \|(\lambda 2)_{\kappa}=2 L\right\rangle_{2}$ \\
\hline \multirow{3}{*}{$\ddot{-}$} & $L-1$ & $-\frac{(2 \lambda+L+5)\left[(\lambda-L+2)\left(\lambda^{2}+5 \lambda+10\right)(L-2)(L+2)\right]^{\frac{1}{2}}}{[4 \lambda(\lambda+3)(\lambda+5) L(2 L+1) \varphi(\lambda+1, L)]^{\frac{1}{2}}}$ \\
\hline & $L$ & $-\frac{\left\{(\lambda-5) A(\lambda, L)+2 \lambda(\lambda+3)(\lambda+4)\left(4 \lambda-L^{2}-L+14\right)-6(\lambda+1)(\lambda+5)(L-1)(L+2)\right\}[(\lambda+2)]^{\frac{1}{2}}}{2 \varphi(\lambda+1, L)\left[2 \lambda(\lambda+3)(\lambda+5)\left(\lambda^{2}+5 \lambda+10\right) L(L+1)\right]^{\frac{1}{2}}}$ \\
\hline & $L+1$ & $\frac{(2 \lambda-L+4)\left[(\lambda+L+3)\left(\lambda^{2}+5 \lambda+10\right)(L+3)(L-1)\right]^{\frac{1}{2}}}{[4 \lambda(\lambda+3)(\lambda+5)(L+1)(2 L+1) \varphi(\lambda+1, L)]^{\frac{1}{2}}}$ \\
\hline \multirow{5}{*}{$\stackrel{n}{\sim}$} & $L \dot{-2}$ & $\frac{\{8(\lambda+3)(2 \lambda+5)+(\lambda-5) L(L-1)\}[(\lambda+2)(\lambda-L+2)(\lambda+L+1)(L-3)(L-2)(L+1)(L+2)]^{\frac{1}{2}}}{\left[4 \lambda(\lambda+3)(\lambda+5)\left(\lambda^{2}+5 \lambda+10\right)(L-1) L(2 L-1)(2 L+1) \varphi(\lambda+1, L) \varphi(\lambda+1, L-2)\right]^{\mathrm{t}}}$ \\
\hline & $L-1$ & $\frac{\left\{3\left(11 \lambda^{2}+55 \lambda+10\right)-\left(\lambda^{2}+5 \lambda+70\right) L^{2}+8(2 \lambda+5) L\right\}[(\lambda-L+2)(L-2)(L+2)]^{\frac{1}{3}}}{\left[4 \lambda(\lambda+3)(\lambda+5)\left(\lambda^{2}+5 \lambda+10\right)(L-1) L(L+1)(2 L+1) \varphi(\lambda+1, L)\right]^{\frac{1}{2}}}$ \\
\hline & $L$ & $\frac{\left\{(\lambda-5) C(\lambda, L)-2 \lambda(\lambda+3)(\lambda+4) B(\lambda, L)+2(\lambda+1)(\lambda+5)(L-1)(L+2)\left(4 L^{2}+4 L-21\right)\right\}[(\lambda+2)]^{\frac{1}{2}}}{2 \varphi(\lambda+1, L)\left[6 \lambda(\lambda+3)(\lambda+5)\left(\lambda^{2}+5 \lambda+10\right) L(L+1)(2 L-1)(2 L+3)\right]^{\frac{1}{2}}}$ \\
\hline & $L+1$ & $-\frac{\left\{3\left(11 \lambda^{2}+55 \lambda+70\right)-\left(\lambda^{2}+5 \lambda+70\right)(L+1)^{2}-8(2 \lambda+5)(L+1)\right\}[(\lambda+L+3)(L-1)(L+3)]^{\frac{1}{2}}}{\left[4 \lambda(\lambda+3)(\lambda+5)\left(\lambda^{2}+5 \lambda+10\right)(L+2)(L+1) L(2 L+1) \varphi(\lambda+1, L)\right]^{\ddagger}}$ \\
\hline & $L+2$ & $\frac{\{8(\lambda+3)(2 \lambda+5)+(\lambda-5)(L+1)(L+2)\}[(\lambda+2)(\lambda+L+3)(\lambda-L)(L+4)(L+3) L(L-1)]^{\frac{1}{2}}}{\left[4 \lambda(\lambda+3)(\lambda+5)\left(\lambda^{2}+5 \lambda+10\right)(L+2)(L+1)(2 L+1)(2 L+3) \varphi(\lambda+1, L) \varphi(\lambda+1, L+2)\right]^{\frac{1}{2}}}$ \\
\hline
\end{tabular}

$p(\lambda, L)=2(\lambda+1)^{2}-L(L+1)$,

$4(\lambda, L)=2(\lambda+1)^{2}\left(L^{2}+L-4\right)+2(\lambda+1)\left(3 L^{2}+3 L-14\right)-\left(L^{4}+2 L^{3}-9 L^{2}-10 L+32\right)$,

$B(\lambda, L)=2(\lambda+4)(L+4)(L-3)+7 L^{2}+7 L+6$,

$\tau(\lambda, L)=4(\lambda+1)^{3}(L+4)(L-3)+18(\lambda+1)^{2}(L+4)(L-3)-2(\lambda+1)\left(L^{4}+2 L^{3}-34 L^{2}-35 L+186\right)$

$-\left(5 L^{4}+10 L^{3}-109 L^{2}-114 L+288\right)$. 


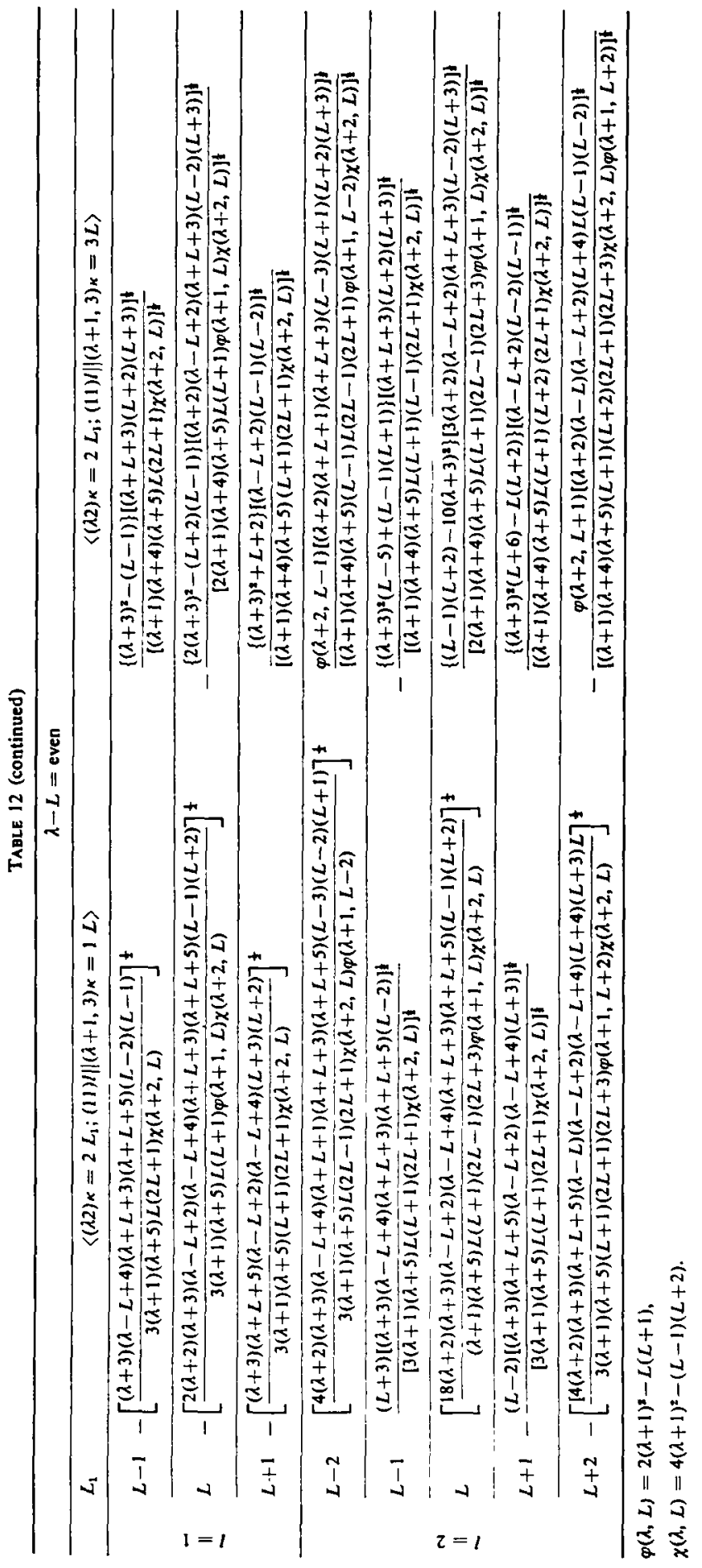




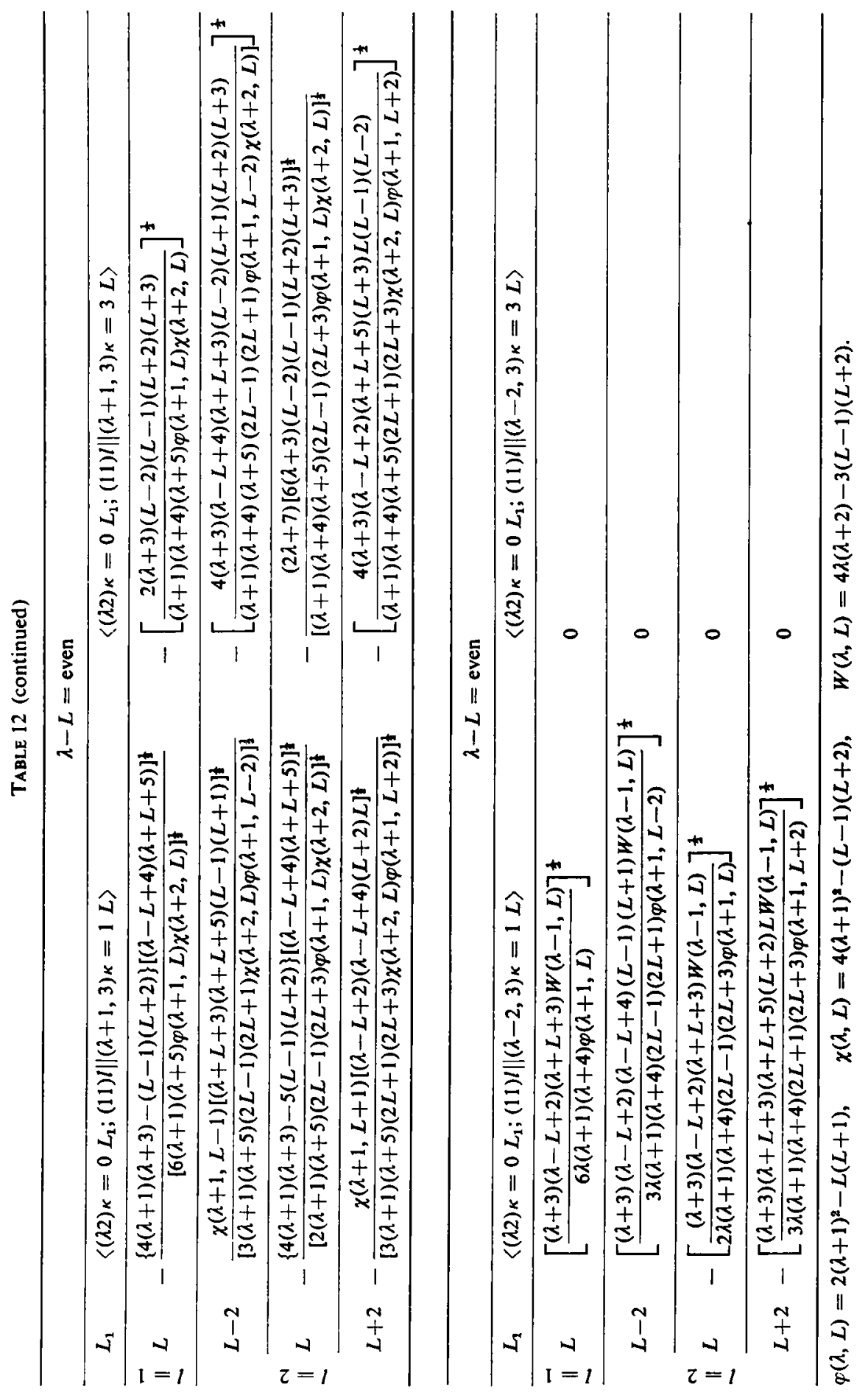




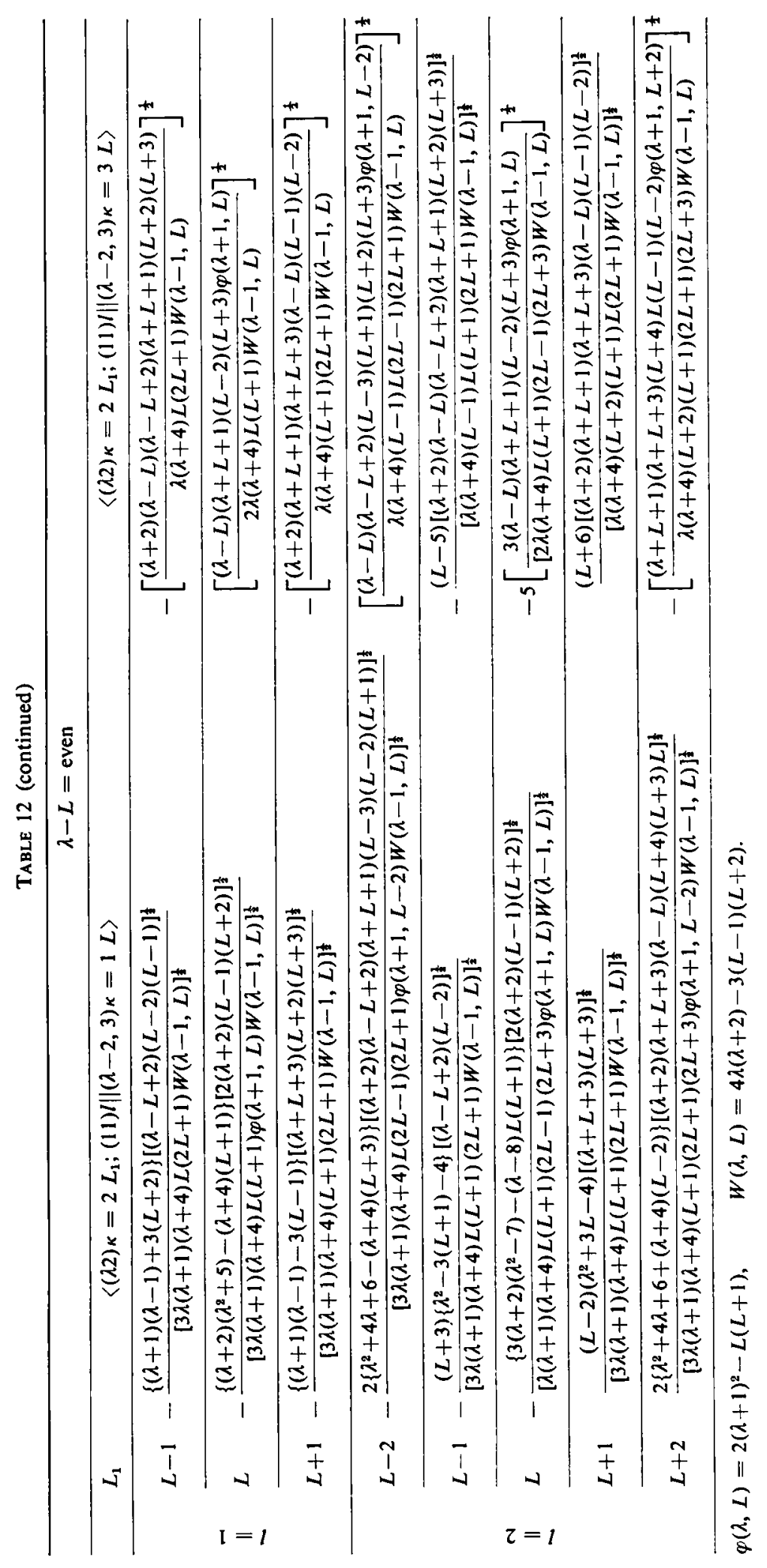


TABle 13

$\left\langle(\lambda 0) L_{1} ;(40) l \|(\lambda+4,0) L\right\rangle$

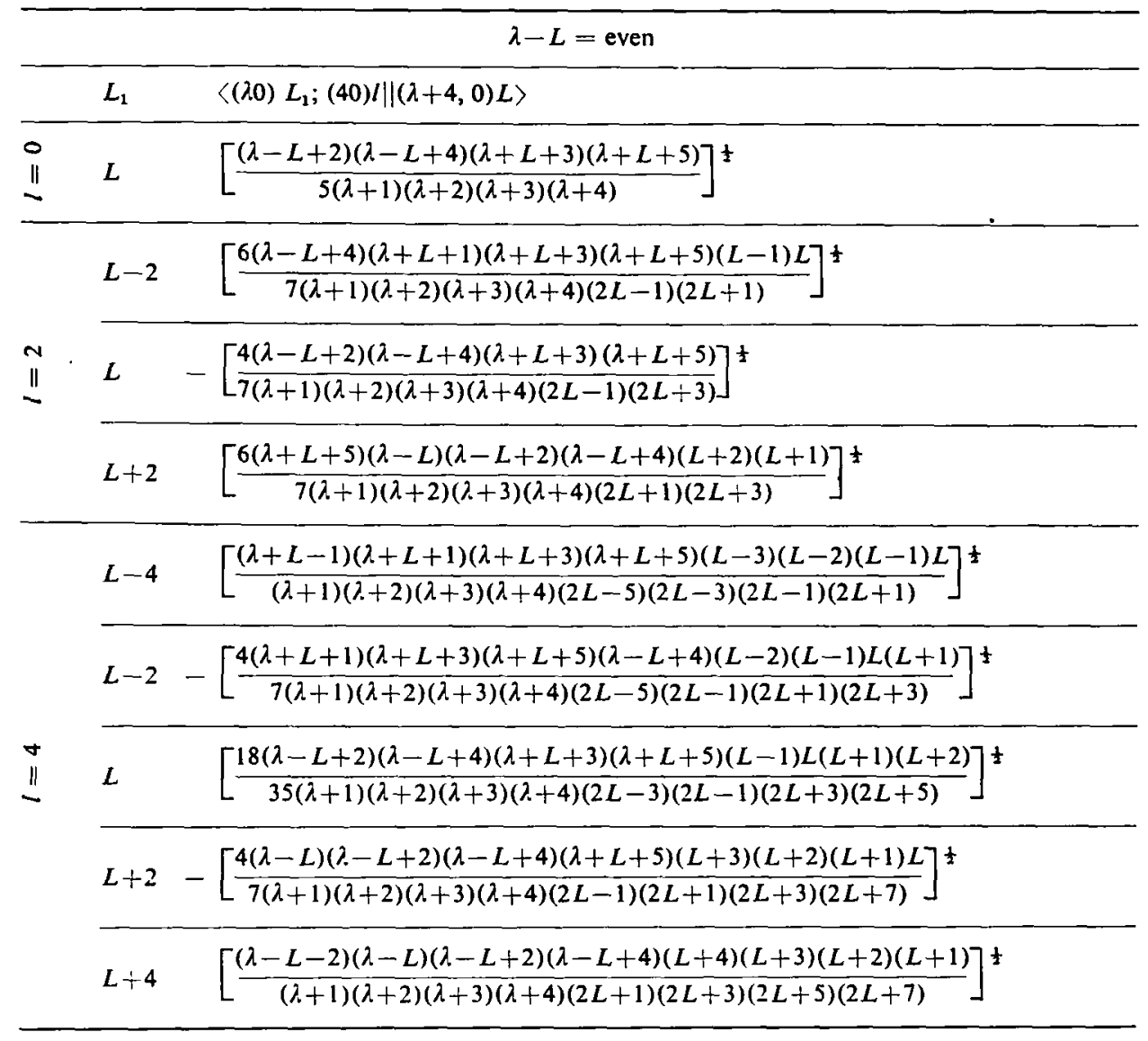

\section{References}

1) J. P. Elliott, Proc. Roy. Soc. A245 (1958) 128, 562

2) J. P. Elliott and M. Harvey, Proc. Roy. Soc. A272 (1963) 557

3) K. T. Hecht, Nucl. Phys. 62 (1965) I

4) T. Engeland, Nucl. Phys. 72 (1965) 67

5) M. K. Banerjee and C. A. Levinson, Phys. Rev. 130 (1963) 1036, 1064

6) D. Koltun, Phys. Rev. 124 (1961) 1162

7) D. M. Brink and G. F. Nash, Nucl. Phys. 40 (1963) 608

8) J. Flores and M. Moshinsky, Nucl. Phys. A93 (1967) 81 and refs. there

9) 1. M. Gelfand and M. L. Tseitlin, Dokl. Akad. Nauk SSSR 71 (1950) 825

10) L. C. Biedenharn, J. Math. Phys. 4 (1963) 436

11) M. Moshinsky, J. Math. Phys. 4 (1963) 1128

12) Selected topics in nuclear spectroscopy, ed. by B. J. Verhaar (North-Holland Publ. Co., Amsterdam, 1964) p. 51

13) J. J. deSwart, Revs. Mod. Phys. 34 (1962) 916 
14) V. Bargmann and M. Moshinsky, Nucl. Phys. 23 (1961) 177

15) H. Horie, J. Phys. Soc. Japan 19 (1964) 1783

16) Y. Akiyama, private communication

17) G. F. Simmons, Introduction to topology and modern analysis (McGraw-Hill Book Co., New York, 1963)

18) G. Neely, University of Michigan dissertation, to be published

19) E. U. Condon and G. H. Shortly, The theory of atomic spectra (Cambridge University Press, 1935)

20) G. Racah, Group theory and spectroscopy Lecture notes, Princton (1951); CERN reprint 61-8 (1961)

21) A. P. Stone, Proc. Cambridge Phil. Soc. 57 (1961) 460

22) A. R. Edmonds, Angular momentum and quantum mechanics (Princeton University Press, 1957)

23) M. E. Rose, Elementary theory of angular momentum (John Wiley, New York, 1957)

24) M. Iosifescu and F. Stancu, Nucl. Phys. B1 (1967) 471 